\title{
WestVirginiaUniversity
}

THE RESEARCH REPOSITORY @ WVU

Graduate Theses, Dissertations, and Problem Reports

2021

\section{Essays in Spatial and Regional Economics}

Mohamed Shabani Kariburyo

West Virginia University, mk0138@mix.wvu.edu

Follow this and additional works at: https://researchrepository.wvu.edu/etd

Part of the Agricultural and Resource Economics Commons

\section{Recommended Citation}

Kariburyo, Mohamed Shabani, "Essays in Spatial and Regional Economics" (2021). Graduate Theses, Dissertations, and Problem Reports. 10271.

https://researchrepository.wvu.edu/etd/10271

This Dissertation is protected by copyright and/or related rights. It has been brought to you by the The Research Repository @ WVU with permission from the rights-holder(s). You are free to use this Dissertation in any way that is permitted by the copyright and related rights legislation that applies to your use. For other uses you must obtain permission from the rights-holder(s) directly, unless additional rights are indicated by a Creative Commons license in the record and/ or on the work itself. This Dissertation has been accepted for inclusion in WVU Graduate Theses, Dissertations, and Problem Reports collection by an authorized administrator of The Research Repository @ WVU.

For more information, please contact researchrepository@mail.wvu.edu. 


\title{
Essays in Spatial and Regional Economics
}

\author{
Mohamed Shabani Kariburyo \\ Dissertation submitted to the \\ Davis College of Agriculture,Natural Resources and Design \\ at West Virginia University \\ in partial fulfillment of the requirements \\ for the degree of \\ Doctor of Philosophy \\ in \\ Natural Resource Economics \\ Alan Collins, Chair \\ Paul Kinder \\ Xiaoli Etienne \\ Lauri Andress \\ Division of Resource Economics and Management \\ Morgantown, West Virginia \\ 2021
}

Keywords: Quasi-Experimental Methods, Spatial Analysis, Spatial Econometrics, Rural Food Access, Public Health, Economic Development, Foreign Direct Investments, Africa, Local versus Regional Economics, Biofuel Production, Land Use Changes (LUCs), Policy Evaluation.

Copyright 2021 Mohamed Shabani Kariburyo 


\section{Abstract \\ Essays in Spatial and Regional Economics \\ Mohamed Shabani Kariburyo}

This dissertation consists of three essays in spatial and regional economics. In the first essay, hot and cold spots for food providers are determined throughout the state of West Virginia and then those places are used in a quasi-experimental method (entropy balancing) to study the effects of hot and cold spots on diabetes and obesity rates. Although hot spots are found to have lower rates of chronic diseases than non-hot spots and cold spots have higher rates of chronic diseases than non-cold spots - the situation is complicated. For example, the findings of income-induced chronic disease rates in urban areas, where most hot spots are located, is evidence for "food swamps" in these areas. Conversely, cold spots (located mainly in rural areas) have higher rates of chronic diseases which are attributed to a combination of access to food providers along with lacking the means (i.e.,income for low-income households) to form healthier habits. In the second essay, the motives of both traditional (American, Dutch, French) and Chinese Foreign Direct Investments (FDI) in Africa are examined and then quantified to what extent Chinese FDIs spatially crowd in or crowd out other traditional FDIs. Spatial dynamic panel models are implemented where the colonial heritage of African countries is used to spatially link countries due to similar socio-economic and institutional settings.French and Dutch Multinational Enterprises (MNEs) in Africa are found to opt for vertical integration strategies while Chinese MNEs serve as export platforms to third country markets. No spatial interdependencies of American investments are found in Africa. Furthermore, Chinese investments are observed to crowd out American and Dutch investments. While no crowd out/in effects are found between Chinese and French FDI, the conclusion is that the two countries are competing in Africa. Finally, the third essay involves localized effects of ethanol plants in the states of Indiana and Illinois on enrolled Conservation Reserve Program (CRP) land, and total planted corn acreage after passage of the 2007 Energy Independence and Security Act with its implementation of Renewable Fuel Standards (RFS) mandating ethanol in gasoline. Using a Difference-in-Difference (DID) method at the state level provides confusing global effects i.e. the results of this model show that both enrolled CRP and total planted corn acres increased after this mandate. However, when a Synthetic Control Method (SCM) is utilized to treat each ethanol plant as a case study, spatial and temporal post-ethanol mandate effects are shown to be heterogeneous. The conclusion is that the global findings from a DID model are misleading since they show acreage increases, whereas, the localized effects found using a SCM are more suited to understanding the heterogenous land-use changes that occurred at the county level due to RFS mandates. 


\section{Acknowledgements}

I would like to dedicate this thesis to my parents, Mugabekazi Laurence and Dr. Juma Mohamed Kariburyo. Thank you for all the sacrifices that you have made for me to get to this stage of higher education.I love and appreciate you. You guys are the real MVPs.

To my brothers and sisters as well as their spouses, Ali and Christelle, Hamim and Amida, Furaha and Guven, Amida and Igor, thank you from the bottom of my heart for everything that you have done in supporting me to get to this stage. To my nephews and nieces, I could not go without thanking you, since you guys are the comedians of the family. Thank you for all the laughers that you have offered me throughout this journey.

To my fiancee Grace, although I met you toward the end of this journey, I am not sure if I would have made it without your consistent love and care. Thank you for everything that you do in my life. I love you, and I can't wait to see where the future takes us.

I would like to thank the people who have supported me during my time at West Virginia University. First, I would like to thank my Advisor Dr. Alan Collins from the bottom of my heart. Thank you for accepting me into the NRE program, for mentoring me, for pushing me, and for being patient with me throughout my Ph.D.. Having you in my corner gave me the confidence I needed to complete my Ph.D..

I would like also to thank Dr. Paul Kinder, the co-chair of my dissertation, and the WVU Natural Resource Analysis Center for supporting my education throughout my PhD program. Thank you for trusting me, for always providing comments on my ongoing research, and for being one of the coolest Educator I have ever met.

To Dr. Lauri Andress, I would like to thank you for accepting me into the food 
desert project, for your leadership on this project, and for consistently mentoring me on how to navigate grad school life. Your advice were/and are still truly appreciated. Thank you so much for all that you have done for me.

To Dr. Xiaoli Etienne, thank you so much for your time and advice. You never failed to provide me with detailed comments on my work, despite being busy with your academic duties. I would like also to thank few scholars that have impacted my higher education life. Thank you to Dr. David Switzer, Dr. David Cuberes, and Dr. Christine Moser for mentoring and inspiring me to get to the Ph.D..

I am grateful to have had the opportunity to interact and learn from talented graduate students while at WVU; in particular, Kuan Ming Huang, Douglas Mugabe, Maher Mekky, Ritika Khurana, Syedul Islam, Alex Scarcioffolo, Stacia Harper, Sara Farhangdoost, and Michael O' Connor. And of course my NRAC people, Samuel Bearinger, Lucas Kinder, and Angela Klein Heintz.

Finally, I would like to thank my friends Mrisho, Melissa, Mika, Florian, Christophe, Parfait, Elvis, Alain, Michael Endale, Amina and Vova for your consistent support, and for making me feel that I was still a sociable person throughout this journey. I am truly thankful for your friendship. I also would like to thank everyone who helped me directly or indirectly throughout this journey. Even if I am not able to list everyone, please accept my sincere appreciation. 


\section{Contents}

Acknowledgements iii

List of Figures vii

List of Tables viii

1 Introduction $\quad 1$

1.1 Contributions . . . . . . . . . . . . . . . . . . 1

2 Place Effects and Chronic Disease Rates in a Rural State: Evidence from a Triangulation of Methods $\quad 4$

2.1 Introduction . . . . . . . . . . . . . . . . . . . . 4

2.2 Literature Review . . . . . . . . . . . . . . . . . . . . . 7

2.3 Data and Methods . . . . . . . . . . . . . . . . 9 9

2.3.1 Hot and Cold Spots (Place Effects) . . . . . . . . . . . . 10

2.3.2 Entropy Balancing . . . . . . . . . . . . . . . . . 13

2.3 .3 Data . . . . . . . . . . . . . . . 15

2.4 Results . . . . . . . . . . . . . . . . . . . 16

2.4.1 Descriptive Statistics . . . . . . . . . . . . . . . . . 16

2.4 .2 Results . . . . . . . . . . . . . . . . . 17

2.4.3 Sensitivity Analysis . . . . . . . . . . . . . . . . . 19

2.5 Discussion . . . . . . . . . . . . . . . . . 20

2.6 Limitations . . . . . . . . . . . . . . . . . . . . 22

2.7 Figures and Tables . . . . . . . . . . . . . . . . . . . 23

3 Colonial Heritage, Traditional, and Chinese Foreign Direct Investments in Africa: A Dynamic Spatial Interdependence Analysis $\quad 38$

3.1 Introduction . . . . . . . . . . . . . . . . . . . . 38

3.2 Literature Review . . . . . . . . . . . . . . . . . . . . . 41

3.2 .1 Chinese Finances in Africa . . . . . . . . . . . . . . . . 42

3.2 .2 Spatial Interdependence of FDI . . . . . . . . . . . . . . . . . 43

3.3 Empirical Methods and Data . . . . . . . . . . . . . . . 45

3.3 .1 Data ....................... 45

3.3.2 Empirical Strategy . . . . . . . . . . . . . . . . . 45 


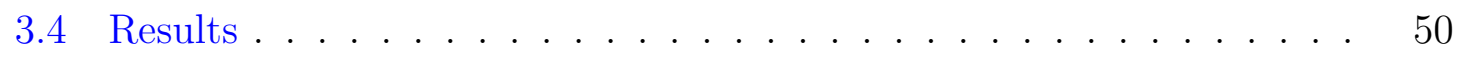

3.4.1 Distribution and Trends of Chinese, Dutch, French and American FDI Flows . . . . . . . . . . . . . . . 50

3.4.2 The Spatial Interdependence of Chinese, Dutch, French and American FDI Flows . . . . . . . . . . . . . 51

3.4.3 Spatial Crowding In and Crowding Out of Chinese FDIs on Traditional FDIs . . . . . . . . . . . . . 56

3.4.4 Sensitivity Analysis . . . . . . . . . . . . . . . . 57

3.5 Conclusion and Implications . . . . . . . . . . . . . . . . . . . . 60

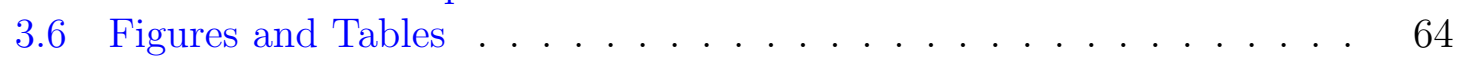

4 The Localized Impacts of Local Ethanol Production on Land Use $\begin{array}{ll}\text { Changes: Evidence from Illinois and Indiana } & \mathbf{7 4}\end{array}$

4.1 Introduction . . . . . . . . . . . . . . . . . . . . 74

4.2 Related Literature . . . . . . . . . . . . . . . . . . . 77

4.3 Empirical Strategies _. . . . . . . . . . . . . . . . . . 80

4.3.1 Case Studies, Data and States Estimates . . . . . . . . . . 80

4.3.2 Synthetic Control Analysis . . . . . . . . . . . . . 83

4.4 Results . . . . . . . . . . . . . . . . . 86

4.4 .1 Post-Effects In Indiana and Illinois . . . . . . . . . . . . 86

4.4.2 Jay County, IN (Outcome: Enrolled CRP Acres) . . . . . . . . 89

4.4.3 Jasper County, Indiana (Outcome: Enrolled CRP Acres and

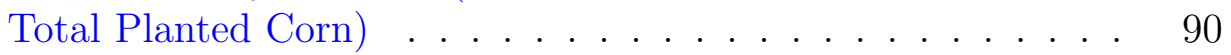

4.4.4 Montgomery County, IN (Outcome: Enrolled CRP Acres) and Shelby County, IN (Outcome: Total Planted Corn) . . . . . . 91

4.4.5 Ogle County, IL (Outcome: Enrolled CRP Acres) . . . . . . . 92

4.4.6 Tazewell County, IL (Outcome: Enrolled CRP Acres) . . . . . 92

4.4.7 Macon and Crawford Counties, IL (Outcome: Total Planted Corn $\ldots \ldots \ldots \ldots \ldots 9 . \ldots \ldots$

4.5 Discussion . . . . . . . . . . . . . . . . . . . . . . . 93

4.6 Conclusion . . . . . . . . . . . . . . . . . . . . . . . 96

4.7 Figures and Tables . . . . . . . . . . . . . . . . . . 99

5 Concluding Remarks $\quad 120$

6 Appendices $\quad 122$ 


\section{List of Figures}

2.1 Food Retail Distribution in West Virginia. . . . . . . . . . . . . . . 23

2.2 Hot Spots and Cold Spots of Food Providers in West Virginia. . . . . 24

2.3 Food Retail Distribution and Poverty Rates. . . . . . . . . . . . . . . 25

2.4 Heat Map of Food Providers in West Virginia. . . . . . . . . . . . . 26

3.1 Trends of French, Dutch, American, and Chinese FDI (in millions dollars) 64

3.2 FDI Stocks in Africa (in billions Dollars) . . . . . . . . . . . . 64

3.3 FDI Percentile Distribution in Africa . . . . . . . . . . . . . . . 65

4.1 Production Volume of Fuel Ethanol in the United States 2000-2020 (in

Million Gallons) . . . . . . . . . . . . . . . . . . 99

4.2 Ethanol Producing States . . . . . . . . . . . . . . . . . . 100

4.3 Treated Counties in Illinois . . . . . . . . . . . . . . . . . . . . 103

4.4 Treated Counties in Indiana . . . . . . . . . . . . . . . . . . 103

4.5 Parallel Trends for Illinois and Indiana . . . . . . . . . . . . . . . . . 104

4.6 Donor Weights . . . . . . . . . . . . . . . . . 105

4.7 Jay, Indiana . . . . . . . . . . . . . . . . . . . . . . 111

4.8 Jasper, Indiana (CRP Acres) . . . . . . . . . . . . . . . . . . . . . . . . . . . . . . . . . . . 112

4.9 Jasper, Indiana (Corn Acreage) . . . . . . . . . . . . . . . . . . . 113

4.10 Montgomery, Indiana . . . . . . . . . . . . . . . . . . . . . 114

4.11 Shelby, Indiana . . . . . . . . . . . . . . . . . . 115

4.12 Ogle, Illinois . . . . . . . . . . . . . . . . . . . . . . . 116

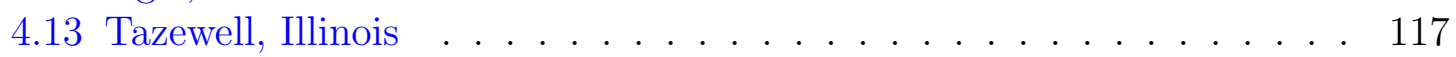

4.14 Macon, Illinois . . . . . . . . . . . . . . . . . . . . . . 118

4.15 Crawford, Illinois . . . . . . . . . . . . . . . . . . 119 


\section{List of Tables}

2.1 Variable Descriptive Statistics for 2016 Data ( $t$-test in parentheses). . 27

2.2 Covariate Balancing. . . . . . . . . . . . . . . . . 28

2.3 The Impacts of Hot and Cold Spots on Diabetes Rates. . . . . . . . . 29

2.4 The Impacts of Hot and Cold Spots on Obesity Rates. . . . . . . . . 29

2.5 The Impacts of Urban and Rural Hot (and Cold) Spots on Obesity (2016). . . . . . . . . . . . . . . . . 30

2.6 The Impacts of Urban and Rural Hot (and Cold) Spots on Diabetes (2016). . . . . . . . . . . . . . . . . . 31

2.7 Propensity Score Weighting Results for Diabetes. . . . . . . . . . . . 32

2.8 Propensity Score Weighting Results for Obesity. . . . . . . . . . . . . 33

2.9 Kernel Matching Results for Diabetes. . . . . . . . . . . . . . . . . . . 34

2.10 Kernel Matching Results for Obesity. . . . . . . . . . . . . . . . 35

2.11 The Impacts of Urban and Rural Hot (and Cold) Spots on Diabetes

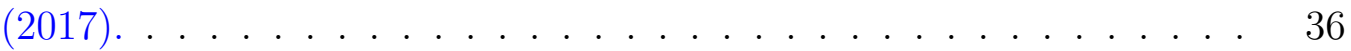

2.12 The Impacts of Urban and Rural Hot (and Cold) Spots on Obesity (2017) . . . . . . . . . . . . . . . . . 37

3.1 Foreign Direct Investments Motives . . . . . . . . . . . . . . . . 66

3.2 Data and Sources . . . . . . . . . . . . . . . . 67

3.3 African Countries and Colonial Historical Ties . . . . . . . . . . . . 68

3.4 Summary Statistics . . . . . . . . . . . . . . . . . 68

3.5 Determinants of FDI, Fixed Effects Panel Regression . . . . . . . . . 69

3.6 Spatial Interdependence of FDI Flows, SDM with Historical Colonial Ties Weight Matrix . . . . . . . . . . . . . . . . . 70

3.7 Spatial Crowding In and Crowding Out of FDI Flows, SDM with Historical Colonial Ties Weight Matrix . . . . . . . . . . . . . . 71

3.8 Spatial Interdependence of FDI Flows, SAR with Historical Colonial Ties Weight Matrix . . . . . . . . . . . . . . . . . . 72

3.9 Spatial Interdependence of FDI Flows, SDM with Distance Weight Matrix . . . . . . . . . . . . . . . . . . 73

4.1 Difference In Difference at the State Level . . . . . . . . . . . . . . . 101

4.2 Ethanol Plants Starting Operation Year . . . . . . . . . . . . . . . 102 
4.3 Post-treatment Effects for Statistically Significant Counties in Illinois 106

4.4 Post-treatment Effects for Statistically Significant Counties In Indiana 107

4.5 Post/Pre RMSPE Ratio . . . . . . . . . . . . . . . . . . . . . . . 108

4.6 Predictor Balance with Planted Corn Acres as the Outcome . . . . . 109

4.7 Predictor Balance with Cumulative CRP Acres as the Outcome . . . 110

6.1 Post-treatment Effects for Statistically Insignificant Counties in Indiana (Enrolled CRP Acres) . . . . . . . . . . . . . . . . . . . 123

6.2 Post-treatment Effects for Statistically Insignificant Counties in Indiana (Total Planted Corn Acres) . . . . . . . . . . . . . . . . . . . . 124

6.3 Post-treatment Effects for Statistically Insignificant Counties in Illinois (Enrolled CRP Acres) . . . . . . . . . . . . . . . . . . 125

6.4 Post-treatment Effects for Statistically Insignificant Counties in Illinois (Total Planted Corn Acres) . . . . . . . . . . . . . . . . . . 126 


\section{Chapter 1}

\section{Introduction}

This thesis comprises three empirical essays that examine topics in health, environmental and development economics while explicitly accounting for spatial dimensions in all of them. It draws and contributes to the literature's in spatial health economics, spatial interdependence of Foreign Direct Investments, and the local versus global environmental effects of biofuel production. The common theme to all the three essays is the fact that they all fit within the spatial and regional economics umbrella. Proost and Thisse (2019) define spatial economics as bringing location, transport, and land into economics. I aim to show in this dissertation that spatial economics is more than just bringing these three variables in economics. The main goal of this dissertation is to show how many of today's real-world problems can be seen as having some spatial aspect and are in need of an integrated analysis.

\subsection{Contributions}

While many studies have tackled the notion of a food desert in rural America, few take into account the different complexities of the food landscapes in rural America. In Chapter 2, we combined a notion from spatial statistics, "Hot Spot and Cold Spot" with a quasi-experimental method, Entropy Balancing, to study how diabetes and obesity rates differ in places that have a higher number of food providers compared 
to those that have little to none. By using the location of different stores, this study demonstrates how one can merge socioeconomic variables, health outcomes, and these locations to get an idea of how spatial differences in food providers influence health outcomes. By capitalizing on public access to these data sets, Chapter 2 shows a methodology that can be used at the state level to inform decision-makers on the differences in health outcomes created by spatial inequities between food providers.

In Chapter 3, we add to the literature of the spatial interdependence of Foreign Direct Investments (FDI), by studying how traditional investors (France, the Netherlands, and the United States) in Africa spatially interact with a non-traditional investor (China). In this literature, the inverse distance weight matrix is widely used to capture spatial interlinkages between the host African countries. However, the way this weight matrix has been standardized in the past has created a debate around this weight matrix; with some studies choosing to normalize this weight matrix by row-normalizing it, despite the shortcomings that this route creates (Lemi et al., 2021; Blonigen et al., 2007; Nwaogu and Ryan, 2014); and others choosing a normalization procedure where each element of the weight matrix is divided by its largest eigenvalue (Regelink and Elhorst, 2015). In this Chapter, we take advantage of the rich African history, by creating a weight matrix that takes into consideration African countries' colonial roots. We argue that the historical colonial ties of African countries still shape the current African economic systems. We find Chinese, Dutch, American, and French FDIs motives while using this historical colonial tie weight matrix, and compare and contrast our results with previous studies.

Finally, Chapter 4 contributes to the literature that tries to understand the effects of ethanol plants on land-use changes. We use the ethanol mandate of 2007 as a policy intervention to implement the Synthetic Control Method (SCM) of (Abadie and Gardeazabal, 2003; Abadie et al., 2015; Abadie, 2021) and study how the enrolled Conservation Reserve Program (CRP) land and total planted corn acres in counties that had an ethanol plant in Illinois and Indiana reacted. While the literature on ethanol production and land use changes has been extensive, this study tries to use 
the SCM to retrieve post-RFS effects on a case-by-case basis, a work that previous studies fail to do. Understanding the local impacts of ethanol production on CRP and corn production land is an important question for local land use policymakers; since, CRP provides important environmental services, which might be lost as conservation land returns to commercial crop production (Ifft et al., 2019). Furthermore, more land under corn cultivation would also create issues of land use at the extensive and intensive margin, which are both harmful to the environment. By providing local post-ethanol mandate effects, this study highlights how studying the effects of this policy on a case by case basis help detect cases of land use at the extensive margin, unlike the single regional or country wide point estimates that previous studies tend to offer (Chen and Khanna, 2018; Li et al., 2019; Searchinger et al., 2008).

A revised version of the first essay that I co-authored with Dr. Alan Collins, Dr. Lauri Andress, and Dr. Paul Kinder is published in the International Journal of Environmental Research and Public Health 2020, 17(18), 6676. A version of the second essay is being prepared to be submitted in Economic Modelling with my coauthors Dr. Xiaoli Etienne and Dr. Shishir Shakya. The third essay is still a working paper. The remainder of this thesis discusses every Chapter in detail. 


\section{Chapter 2}

\section{Place Effects and Chronic Disease Rates in a Rural State: Evidence from a Triangulation of Methods}

\section{$2.1 \quad$ Introduction}

Poor diet is a modifiable risk factor for obesity and diabetes. Contributing to substandard nutrition, the food environment is also thought to be a primary driver of poor diet. Health advocates, community leaders, and researchers are worried that food environment problems, and poor diets in general, may be more severe in certain low income and rural American communities because these areas have limited access to affordable and nutritious foods. A primary concern is that some poor or rural areas do not have access to supermarkets, grocery stores, or other food retailers that offer the large variety of foods needed for a healthy diet (for example, fresh fruits and vegetables, whole grains, fresh dairy, and meat products) (Andress and Fitch, 2016; Andress and Hallie, 2017; Andress, 2017; Jilcott Pitts et al., 2012). Instead, individuals in these areas may be more reliant on food retailers or fast food restaurants that only offer more limited varieties of foods. It is hypothesized that the relative lack of access to full-service grocery stores and the easier access to fast and convenience 
foods may be linked to poor diets, and ultimately, to obesity and other diet-related diseases (Caspi et al., 2012; Creel et al., 2008).

It was this concern that led Congress, in the Food, Conservation, and Energy Act of 2008 (hereafter referred to as the 2008 Farm Bill) to instruct the U.S. Department of Agriculture (USDA) to conduct a 1-year study assessing the extent of the problem of "food deserts." More specifically, the remit in the 2008 Farm Bill called upon the USDA to study the limited access to food, characteristics, and causes of limited access, the effects limited access has on local populations and recommendations for addressing the causes and effects of limited access. As a result, numerous researchers have tried to empirically measure the association between the food environment and obesity and diabetes during this past decade. These studies have investigated whether living in a "food desert" or "food swamp" caused higher rates of these chronic diseases or increased nutritional inequality between the wealthier and the less fortunate population (Cooksey-Stowers et al., 2017; Cummins et al., 2014; Allcott et al., 2019). Food deserts are often described as residential areas with limited vendors of affordable and healthy foods(Cooksey-Stowers et al., 2017). In comparison, food swamps are areas with a higher number of unhealthy vendors, such as convenience stores and fast food restaurants (Phillips and Rodriguez, 2020). Despite this surge of researchers, most studies still failed to prove the causal claim that underserved areas are associated with an increased risk of chronic diseases, with few exceptions (Cooksey-Stowers et al., 2017; Phillips and Rodriguez, 2020). Nonetheless, federal and local governments continue to support the supply-side through initiatives such as the Healthy Food Financing Initiative and the Agricultural Act of 2014. ${ }^{1}$.

It is in this context that some researchers have started to question the metaphor of a "food desert" and its applicability in different geographical entities in the United States. This argument states that studies in this field should not just measure the food

\footnotetext{
${ }^{1}$ Since 2011, the healthy food financing initiative has been providing subsidies to grocery stores, farmers markets, and other actors working in underserved areas. The Agricultural Act of 2014 allocated about 125 millions in federal funds to support access to healthy food in underserved areas (Allcott et al., 2019)
} 
environment as binary - areas where people live in a food deserts or food swamps, and areas where people do not live in these regions. Instead, it is important to consider how locals perceive their food environment (Andress and Fitch, 2016; Cummins and Macintyre, 2002). This is a way to include the spatially-explicit factors that individuals have to face on a daily basis. Furthermore, the food environment is believed to be different in urban and rural areas - what is considered a "food swamp" in one area might be a "food oasis" in another (Sharkey et al., 2013). Therefore, to understand the impact of the food environment on chronic diseases, it is crucial to properly define the food environment spatial measure.

In this research, we explicitly account for spatial drivers that may influence the supply of food vendors and study how these places might impact diabetes and obesity rates in West Virginia. This state is an interesting case to study. It leads the nation in obesity rates and is ranked the second-highest in diabetes rates nationally ${ }^{2}$. This study will add to efforts that evaluate and understand how the neighborhood retail food environment contributes to these chronic diseases. In economics and public health literature, this paper is related to articles that studied the causal link between the food environment and chronic diseases (Phillips and Rodriguez, 2020; Currie and Walker, 2011; Allcott et al., 2019; Alviola et al., 2013; Cummins et al., 2014). We combine the hot spot analysis results with a quasi-experimental approach (entropy balancing) to study how diabetes and obesity rates might be impacted by these places (hot and cold spots). To our knowledge, this is the first study to combine results from the hot spot analysis and a quasi-experimental approach to address this issue. First, we wanted to test whether places with an abundance of food providers (hot spots) and those that lack food providers (cold spots) are associated differently with diet-related diseases. Second, we wanted to understand whether the difference in these chronic diseases rates between hot (and cold) spots depends on whether a census tract is urban or rural. Finally, we wanted to test whether there is a significant diet-related

\footnotetext{
${ }^{2}$ based on 2018 statistics provided by the West Virginia Department of Health and Human Resources Bureau for Public Health: https://dhhr.wv.gov/hpcd/data_reports/pages/fast-facts.aspx
} 
disease-income relationship between hot (and cold) spots.

The remainder of the article is organized as follows: in Section 2.2, a literature review on chronic diseases and place-based policies is elaborated; Section 2.3 explains the methods and the data used in this study; Section 2.4 presents the results; Section 2.5 provides some discussion and policy implications; finally, Section 2.6 discusses about the limitations of this research.

\subsection{Literature Review}

Studies associating chronic diseases and the overall food environment across different geographical regions have been surging in economics and public health fields. These studies consider whether neighborhood food vendors play a role in nutritional inequality, and therefore, chronic diseases.

The literature behind the associations between healthy and unhealthy food outlets and chronic diseases has heavily relied on spatial analysis or geographic information system data (Caspi et al., 2012; Allcott et al., 2019; Chen et al., 2016). To examine the association between food deserts (and food swamps) and consumption of snacks/desserts or fruits/vegetables, Hager et al. (2017) combined geocoded home addresses and maps of food deserts/food swamps. They found that adolescent girls living in an unhealthy environment (food swamps) had a higher intake of snacks/desserts than girls not living in these areas. Further tackling the issue of food swamps and food deserts, Cooksey-Stowers et al. (2017) combined commercial street reference, socioeconomic, food environment, and obesity datasets. Their results suggest that food swamps are associated with higher obesity rates, but the absence of a full grocery store shows inconclusive results. Jang and Kim (2018) investigated the intersectionality of race/ethnicity and poverty in terms of spatial access to supermarkets, grocery stores, and convenience stores in the Detroit metropolitan area. They suggest that place-based policies can successfully improve health intervention strategies when implemented by federal, state, and local authorities. 
By further examining nutritional inequalities in food swamps and food deserts, Phillips and Rodriguez (2020) found that the association between hospitalization rates among adults and diabetes followed a curvilinear relationship, plateauing at the saturation point of unhealthy food stores. Using a measure of diabetes incidence, Christine et al. (2015) showed that having resources that support physical activity and healthy diets in the neighborhood can lower the incidence of type 2 diabetes. In most research, the definition of chronic disease measures is clearly specified with the association between food environment and diabetes incidence being analyzed, and the relationship between food swamps and a measure of severity being treated in Phillips and Rodriguez (2020) and Gebreab et al. (2017).

There are also recent articles, using large, nationally representative samples that have started to question the hypothesis that the neighborhood food environment (i.e., supply side factor) reduces nutrition inequality. Allcott et al. (2019) argued that increasing the supply of healthy groceries alone does not reduce nutritional disparities. Using counterfactual simulations, they demonstrated that simply fixing food deserts by adding stores or responding only to supply-side issues achieves merely a ten percent reduction in nutritional inequality. Alternatively, the researchers assert that the remaining ninety percent difference in nutritional inequality might be driven by any number of demand-side factors in their simulations, including education, prices, and/or preferences (Allcott et al., 2019).

Johnson et al. (2014) highlighted a vital trend in the neighborhood food environment literature - most research on this subject tends to be on urban communities and rural communities are mainly left behind. Bailey (2010) sounded the alarm on how traditional grocery stores are becoming scarce in rural America . Using a crosssectional study, Sharkey et al. (2013) showed that in Texas border colonia households, convenience stores were the critical variable that influenced nutrients intakes. The demand for food in rural America relies on many different healthy and unhealthy stores due to the shortages in the supply. Some households depend on dollar stores, big box retailers (e.g., Walmart), convenience stores (e.g., Sheetz), fast food restaurants, and 
farmers markets. Understanding places might be the missing point in solving nutritional and health disparities in these communities. These papers focused on rural areas and identified determinants related to access to healthy and affordable food to improve population health and reduce health inequalities in rural America (Johnson et al., 2014; Sharkey et al., 2013).

Further, studies focusing on either rural or urban areas may differ in findings because of the study area and empirical methodologies. Alviola et al. (2013) found that fast-food restaurants and convenience stores were the main determinants of food deserts in a rural state like Arkansas. Allcott et al. (2019) demonstrated how the addition of a healthy store in certain geographical areas does little in reducing nutritional inequalities. Their national representative data helped them advance some policy implications that could help federal, state, and city officials in their intervention strategies.

Overall, much of the previous research has highlighted the need to provide more evidence on the effect of supply inequities on chronic diseases. The lack of causal effect studies that try to understand the association between chronic diseases and food environment leaves many unanswered questions on this matter. By evaluating how places in a largely rural state impact diabetes and obesity rates, our research aims to pinpoint areas that are at risk of chronic diseases.

\subsection{Data and Methods}

To understand the relationship between West Virginia's food environment (place effects) and chronic diseases, this research focuses on food provider geographic locations with the caveat that while other factors impacting food access certainly exist, they are beyond the scope of this research. Our specific approach to this issue offers a triangulation of methods where we combine results from a spatial analysis method (hot spot analysis) with a matching technique (entropy balancing), and an ordinary least square (OLS) regression to understand whether the extent of food providers' 
presence and/or absence impacts chronic diseases rates in West Virginia. Recent literature reviews suggest that this combination of methodologies to identify the effects of retail food providers on chronic disease rate incidence constitutes a new approach in this literature (Marasteanu and Jaenicke, 2019). ${ }^{3}$

\subsubsection{Hot and Cold Spots (Place Effects)}

To capture the geographic distribution and patterns of food stores in West Virginia, this research utilizes a dataset from the WV Foodlink project ${ }^{4}$ housed at the food justice laboratory within the Department of Geology and Geography at West Virginia University. In collaboration with their community partners, this laboratory has developed a resource hub and learning commons to support a people-centered, resilient food network in West Virginia. These data contain the geolocation of charitable assistance agencies, big box retailers, grocery stores, small box retailers, convenience stores, farmers markets, and undefined SNAP accepting retailers. In this data, we count 68 big box stores, 996 convenience stores, 134 farmers markets, 320 grocery stores, and 587 small box retailers. Toward the end of 2015, the WV Foodlink project verified the validity of these locations by sending participants from this project to the specific site of a food store (or outlet) to confirm their actual latitude and longitude. In this research, food stores geographic coordinates were used as a basis in all spatial analysis conducted.

In the spatial analysis practice, researchers usually use two methods to map clusters: heat maps and hot spot analysis. The heat map as illustrated in Figure 2.4 utilises a color gradient that indicates areas of increasingly higher density. In our map, food vendors tend to cluster around West Virginia larger cities - and it is very difficult to visualize where the population is lacking food vendors - which is why we fo-

\footnotetext{
${ }^{3}$ Marasteanu and Jaenicke (2019) utilized the same logic while studying the economic impact of organic agriculture hot spots in the United States. The main methodological difference between the two studies is that Marasteanu and Jaenicke (2019) computed the hot spot analysis then used the propensity score matching (PSM), whereas our study used the entropy balancing method and perform a sensitivity analysis using the propensity score weighting and the kernel matching method.

${ }^{4}$ (http ://foodlink.wvu.edu/)
} 
cus on the hot spot analysis in this research. Hot spot analysis is a statistical method that assesses geographic clustering. This method is a local indicator of spatial autocorrelations (LISAs) - it uses variations in local spatial autocorrelation compared to surrounding areas, or areas in which clusters of density or intensity are statistically distinct from the neighboring landscape - an option that a heat map does not offer.

Figure 2.3 displays the specific latitudes and longitudes of all the stores in West Virginia. Each point, as described in the legend, represents a specific type of store. Separating between healthy and unhealthy food suppliers is one option that this research could have followed; however, in some areas in West Virginia, families rely on stores that are considered unhealthy to purchase all their fresh produce ${ }^{5}$. To account for these inequities, this research defines a hot spot as an area which has a much higher than average count of food providers, and a cold spot as an area which has a much lower than average count of food providers ${ }^{6}$. Optimized hot spot analysis 7 is used as a spatial data mining tool that asks the data (in our case, latitude and longitude of each specific store) to obtain the settings that yield the optimal hot spot results. It uses the Getis-Ord $G_{i}{ }^{*}$ which works as follows:

$$
G_{i}=\frac{\sum_{j=1}^{n} w_{i, j} x_{j}-X^{b a r} \sum_{j=1}^{n} w_{i, j}}{S \sqrt{\frac{n \sum_{j=1}^{n} w_{i, j}^{2}-\left(\sum_{j=1}^{n} w_{i, j}\right)^{2}}{n-1}}}
$$

where $x_{j}$ is the attribute value for food provider $j ; w_{i, j}$ is the spatial weight between

\footnotetext{
${ }^{5}$ According to the American Healthy Food Financing, West Virginia is facing a growing problem of store closures-leaving behind thousands of families. As a result, families rely more and more on closer stores such as convenience stores or Mom and Pop stores; see https://www.investinginfood.com/ commentary-w-v-projects-try-new-ways-to-deliver-fresh-food-options/

${ }^{6}$ Clusters of stores whose values are atypically high or low compared to the entire sample. Given a chosen level of statistical significance (e.g., $p<0.001$ ), the hypothesis test identifies high (low) value observations surrounded by other high (low) value observations, where the difference between values observed for these identified clusters and those for the surrounding observations is too great to be the result of random chance. For example, Kanawha county, which is in a hot spot, has 30 grocery stores, 5 big box stores, 80 small box stores, 96 convenience stores, and 1 farmers market. Pocahontas county, a cold spot in our analysis, has 7 grocery stores, 0 big box store, 4 small box stores, 1 convenience store, and 0 farmers markets

${ }^{7}$ for more details on this method please see the ArcMap manual: https://desktop.arcgis. com
} 
food providers $i$ and $j$. The choice of a weight matrix is a very important step during the hot spot analysis and in our case, we use an inverse distance weight matrix. This distance-based weight matrix with a distance band of 10 miles between food providers because the USDA defines low food access census tracts as those who have 500 persons and/or at least 33 percent of the population who live more than 10 miles from a supermarket or a large grocery store ${ }^{8}$. Using this type of distance-based weight matrix, a food provider that is farther away, receives less weight in the analysis. $n$ is equal to the total number of features and $X^{\text {bar }}$ is just an average of $n$ attribute values across West Virginia. Lastly, Equation (2) is the square root of the average of the squares of the sum of all attributes minus the average attribute squared.

$$
S=\sqrt{\left.\left(\frac{\sum_{j=1}^{n} x_{j}^{2}}{n}\right)-\left(X^{b a r}\right)^{2}\right)}
$$

The $G_{i}^{*}$ statistic can be thought of as a z-score so that no further statistical calculation is required.

Figure 2.1 clearly shows the highly uneven food environment landscape in West Virginia. The most common stores in West Virginia are convenience and small box retailers which make up about 76 percent of the retailers in the state. This unevenness was the reason why it was important to row standardize this matrix to give each feature the same weight in the analysis. Figure 2.2 shows a map depicting the results of the hot spot analysis. Among the 484 census tracts in West Virginia, those red areas show census tracts that are hot spots of food providers at a 99 percent confidence interval. The light gray areas are census tracts that represent cold spots at a 90 percent confidence interval. Every statistically significant hot or cold spot area was reported (Figure 2.2 displays a legend with the level of statistical significance at the 90, 95, and 99 percent; we used these visual explanations to designate a census tract as hot or cold spots).

Using the results from this map, a census tract level indicator variable is created,

\footnotetext{
${ }^{8}$ https://www.ers.usda.gov/data-products/food-access-research-atlas/documentation
} 
which takes a value of 1 if the census tract is identified as being part of a statistically significant hot or cold spot, and 0 otherwise. To examine the impact of hot and cold spots on chronic diseases, this research characterizes hot and cold spots as being "treatments." It measures the impact of the treatment on a census tract diabetes and obesity rates.

\subsubsection{Entropy Balancing}

A big challenge of this empirical work is to establish an econometric link between the chronic diseases of diabetes and obesity and those places identified as hot or cold spots for food providers. Several endogenous variables might lead a census tract to being identified as a hot or cold spot. To mitigate the potential endogeneity issue that might arise with being in a hot spot or cold spot, we employ a matching technique.

Since the analysis is based on the concept that a hot or cold spot represents a treatment-our measure of interest is the average treatment effect on the treated $A T T$, which is defined as:

$$
A T T=E\left[y_{1} / D=1\right]-E\left[y_{0} / D=1\right]
$$

where $D$, is an indicator variable that is equal to 1 if a census tract is a hot (or cold) spot and 0 otherwise. For a specific census tract, we denote $E\left[y_{0} / D=1\right]$ as the counterfactual - that is, the outcome of a census tract in a hot (or cold) spot would have been realized had they not been positioned in those geographical locations; and $E\left[y_{1} / D=1\right]$ as the outcome after being treated as a hot (or cold) spot. The counterfactual is unobservable in this case; therefore, a suitable proxy to solve for $A T T$ is needed. In the case of a randomized control trial, the average outcome of units not exposed to treatment, $E\left[y_{0} / D=0\right]$, is a proper substitute. However, as discussed before, being in a hot or cold spot, and thus, selection into treatment, could be endogenous (Marcus, 2013; Hainmueller, 2012).

The matching process's key idea is to facilitate a situation of randomization where 
the assignment to the treatment can happen by a chance procedure. The unobserved counterfactual outcome is assigned by matching the treated units with untreated units that are as similar as possible with regard to all pretreatment covariates that are associated with selection into treatment (being in a hot or cold spot) and impact the dependent of variables of interest (diabetes or obesity rates). The estimate representing ATT based on matching can be written as follows:

$$
\operatorname{ATT}(x)=E\left[y_{1} / D=1, X=x\right]-E\left[y_{0} / D=0, X=x\right]
$$

$x$ is a vector of relevant covariates, which are described in the following subsection, $E\left[y_{1} / D=1, X=x\right]$ is the expected outcome for the census tracts that were in the hot (or cold) spots, and $E\left[y_{0} / D=0, X=x\right]$ is the expected outcome for the best matches of the hot (or cold) spots census tracts (Neuenkirch and Neumeier, 2016).

Studies that have investigated the effectiveness of place-based policies have used nearest neighbor matching, propensity score matching, Mahalanobis distance matching, and entropy balancing (Smith and Todd, 2005; Grossman et al., 2019). Marcus (2013) argued that because of the sensitivity to the choice of matching variables, entropy balancing balances the matching covariates more effectively than the propensity score matching. Furthermore, Deb and Vargas (2016) elaborated a summary of the main difference between entropy balancing and the propensity score matching: the former does not require the researcher to first estimate the weights, and then verify whether the treatment and the control covariates balance like the latter does. Hainmueller (2012) showed that entropy balancing is better suited than either the propensity score or Mahalanobis distance matching. Entropy balancing generates a weighting scheme that reweights control observations so that they are perfectly balanced compared to treated individuals based on the mean moments of covariate distribution. After weighting, the treated indicator is orthogonal to covariate moments included in the entropy balancing exercise. Finally, the weights are then 
incorporated in Equation (5) as a survey weight ${ }^{9}$ :

$$
Y_{i}=\beta_{0}+x_{i} \beta+\epsilon_{i}
$$

where $Y_{i}$ is the rate of diabetes or obesity; and $x_{i}$ is a set of independent variables that includes whether a census tract is considered in treatment (hot spots and cold spots), an urban versus rural designation for each census tract, mean household income, and the proportions of the population that are employed in the sectors of agriculture, fisheries, hunting, and mining (AFHM). The AFHM variable was selected because it is important to understand the relationship between these sectors, which have been hit harshly by economic changes, and the food access inequality (Deskins et al., 2018). Finally, Mactaggart et al. (2016) investigated how health disparities in rural settings may be exacerbated by AFHM occupations.

The independent variables of interest include those to test the first set of key hypotheses that diabetes and obesity rates differ in hot (and cold) spots compared to non-hot (and non-cold) spots. Other hypotheses are examined with interaction variables between the treatments and urban, rural, and household income variables. These hypotheses state that urban or rural aspects of census tracts accentuate the treatment differences; and there exist different chronic disease-income relationships at various income levels and across treatment and non-treatment census tracts.

\subsubsection{Data}

Our triangulation of methods employs three publicly available datasets: food store locations (source: WVfoodlink project), socioeconomic variables (source: American Community Survey (ACS)), and health outcomes (source: County Health Rankings and Roadmaps). Given difficulties in finding experimental and observational data for diabetes and obesity at the census tract level, a metric was developed through the

\footnotetext{
${ }^{9}$ one can think about these survey weights or weights as similar to those that are created by the logit model of the propensity score analysis
} 
following steps. The County Health Rankings and Roadmaps provide the data for the percentage of adults aged 20 and above with diagnosed diabetes at the county level. The same source also provides the percentage of adult population (age 20 and older) that reports a body mass index greater than or equal to $30 \mathrm{~kg} / \mathrm{m}^{2}$ which is considered as obese. In this research, the percentages of each county's diabetes and obesity were distributed to each census tracts.

Using previous research on the determinants of neighborhood food environments (Chen et al., 2016; Alviola et al., 2013), the following socioeconomic characteristics were used as covariates to control for the selection into the treatments: number of family households; individuals who were age 25 and above who had a bachelor's degree; estimated vehicles available; households that received food stamp/SNAP benefits in the pasts 12 months; mean household income (adjusted in 2016 dollars); unemployed civilians who are in the labor force; age groups (<19 and $>59)$; race (the proportion of non-white population); and health insurance coverage. Finally, community health centers (CHS) in each census tracts served as a control for health services. These data originated from the West Virginia Primary Care Association.

\subsection{Results}

\subsubsection{Descriptive Statistics}

Table 2.1 shows the sample means of all matching covariates among four groups: (1) hot spots as the treatment group when a census tract is in a hot spot, (2) non-hot spots that are the control group for hot spots, (3) cold spots as the treatment for cold spot analysis, and (4) non-cold spot control census tracts. Note that there are difference columns between the groups along with the corresponding t-test statistics with a null hypothesis of difference equal to zero.

Table 2.2 compares the means of all matching covariates across the treatment groups and the synthetic group (control) obtained via entropy balancing. This table 
illustrates how entropy balancing improves the quality of matching between hot (cold) and non-hot (non-cold). When we compare the realizations of the pre-treatment characteristics of the hot spots and cold spots to those of the control group, the results reveal the efficacy of entropy balancing. After matching, all covariates are perfectly balanced, and no difference remains. Therefore, the control group in the next regression analysis is comprised of credible counterfactual for the sample of census tract that are hot or cold spots.

\subsubsection{Results}

Section 2.7 presents baseline model results to assess the impacts on diabetes and obesity rates from solely the treatment variable of populations residing in either a hot or a cold spot (Tables 2.3 and 2.4). From these models, results consistently show that hot spots are healthier environments with decreases in diabetes and obesity rates compared to non-hot spots, while cold spots increase the rates for both chronic diseases compared to non-cold spots. In addition, both unbalanced and balanced models are estimated to compare the unbalanced and the entropy balanced models. As is conventional in the literature Hainmueller (2012), these results illustrate the overestimated bias observed from the unbalanced models compared to the corrected balanced models. Our interpretation is that by not accounting for covariates, such as the number of vehicles in a census tract, the mean household income, education, and age groups, this absence could have inflated the effect of living in non-treatment census tracts on diabetes and obesity rates.

Our main results consist of eight regression models showing four sets of treatment effects for hot and cold spots, respectively (Tables 2.6 and 2.5). These models examine the impacts of interactions between urban and rural designation with hot spots (columns (1) and (2)) along with the same interaction impacts with cold spots (columns (3) and (4)) on diabetes (Table 2.6) and obesity (Table 2.5) rates. For urban hot spots, both diabetes $(\beta=-0.0084, p$-value $=0.095)$ and obesity $(\beta=-0.0278$, 
$p$-value $=0.004)$ rates are lower than non-hot, urban, and all rural census tracts. The reduction in obesity rates is particularly notable as it reflects a decrease of seven percent from the statewide average of 37.7 percent in 2016. Urban cold spots had mixed impacts on chronic diseases with a small, statistically significant impact on diabetes $(\beta=-0.0061, p$-value $=0.089)$ and a zero statistical impact on obesity rates. For rural census tracts, the treatment of either a hot or cold spot increased both diabetes and obesity rates by 1-3 percentage points relative to urban census tracts and rural, non-hot, or non-cold spots.

In Tables 2.6 and 2.5, we also sought to determine whether diabetes and obesity rates in hot (and cold) spots are impacted differentially by differing household income levels. Within urban areas, the results point out that as income increases for the top household income quartile (75 percent), both diabetes and obesity rates increase within hot and cold spots. Since, as mentioned earlier in this manuscript, the food landscape in West Virginia is driven by convenience stores and small box retailers, these increases in rates could be evidence of food swamps causing chronic diseases (Cooksey-Stowers et al., 2017; Phillips and Rodriguez, 2020).

In urban cold spots, we still found that there is no evidence of a diabetes rate differential among the lower and middle household income quartiles, and their counterparts in urban non-cold spots. However, we found that an increase in earnings of the top household income increases the rate of diabetes in urban cold spots. This result reinforces the view that urban areas are food swamps in West Virginia. In rural census tracts, the treatment-income interaction effect occurs primarily at the 50 percent and lower household income quartiles with statistically significant, negative impacts on chronic disease rates. The largest impacts from the treatment-income effect are on obesity rates. As an example, a one percent change in household income for the population at the 50 percent quartile in rural hot spots results in a 0.0003 percent reduction in obesity rates. When compared to the rural hot spot treatment increase in obesity rates of 0.0185 percentage points, household income at the 50 percent quartile would have to rise over 60 percent to offset the treatment effect of a hot 
spot in a rural area.

\subsubsection{Sensitivity Analysis}

Our sensitivity analysis is decomposed into two parts: first, we relied on the propensity score weighting and the kernel matching method to analyze the sensitivity of our results - using other matching procedures gave us a sense of reassurance in the plausibility of our results. As explained earlier, these two methods are different to the entropy balancing approach because the propensity score weighting assigns to each control a weight that is equal to $(1 /(1-P(X))$ where $P(X)$ is the propensity score; the kernel matching method uses the observed propensity scores to match treatment and control units while using the closest covariates in terms of propensity scores.

Second, we used the data from year $2017(t+1)$ to verify whether our conclusion holds. Since the locations of all food vendors were verified in 2016, we also ran the entropy balancing model for the year of 2017 because most of the covariates used in the matching process were collected from the American Community Survey (ACS) 2014-2018. Thus, this process helps us understand how our estimates change when we look at another year $(t+1)$ that is close to the time period for which we have data on the geolocations of food providers in West Virginia.

There are several similarities between our main model, entropy balancing (2016), and the propensity score weighting, the kernel matching method, and the 2017 results. Using the same logic, i.e., starting with regressions that include the interactions between the different treatments (urban and rural hot (and cold) spots) and the different income quartiles, we still find the same results for both techniques. However, the coefficients differ in magnitude with the entropy balancing method. The results are econometrically encouraging per se - the combination of the hot spot analysis and the entropy balancing is a solid way of understanding how the food environment affects chronic diseases in certain geographical entities; i.e., these results are not sensitive to time. 
Overall, Tables 2.7-2.12, the results of our sensitivity analyses, inform us that obesity and diabetes models hold up well. When comparing the range of treatment coefficient estimates for entropy balancing (2016) to the propensity score weighting, the kernel matching, and the entropy balancing for the year 2017 for hot and cold spots - the signs of almost all the coefficients are the same as in our entropy balancing model (2016). The only difference resides in the magnitudes of the coefficients. However, the entropy balancing results from the 2017 data are almost the same as those from our original models.

\subsection{Discussion}

Our findings are of relevance to policymakers in rural states such as West Virginia. Overall, these results highlight the fact that places in West Virginia with access to different food vendors (hot spots) tend to have chronic disease outcomes associated with food swamps. This finding is not new in the literature. However, most researchers tend to separate between healthy and unhealthy food stores and find that individuals who have access to stores that offer a variety of food are healthier than inhabitants of food deserts (Caillavet et al., 2015). In this research, we found that explicitly accounting for spatial forces (hot and cold spots) is better suited for a region where a food oasis might be the only choice. In a rural state such as West Virginia, some areas depend on convenience stores or Mom and Pop shops for all their groceries ${ }^{10}$. Figure 2.2 shows that large numbers of cold spots are in the eastern part of West Virginia where Randolph, Pocahontas, Webster, and Tucker counties are considered mountainous and largely rural.

Therefore, separating between healthy or unhealthy stores might not do justice to residents of cold spots for example. Investigating whether a store is in an urban or rural area seems to tease out better the chronic disease-income relationship of individuals in that area. Moreover, our results also inform us that socioeconomic

\footnotetext{
${ }^{10}$ https://www. investinginfood. com/
} 
status (SES) plays a primal role, whether you live in a cold spot or a hot spot. On the one hand, these results showed consistent evidence that income growth in the top income quartile increases both chronic diseases within urban hot and cold spots. On the other hand, household income increases among the 50 percent quartile and lower income groups are linked to lower rates of chronic diseases within rural census tracts.

This research examined the differential impacts of income and access to retail food providers in West Virginia. After controlling for socioeconomic variables in hot and cold spots, we found that urban and rural areas in West Virginia are affected differently by the food landscape. First, we found that the household income impacts on chronic diseases among the top earners in both hot and cold spots located in urban areas are essentially zero. Thus, policies that aim to reduce chronic disease rates by improving access to healthy food alone in urban areas of West Virginia might not work as the problem could be more related to diet preferences than food access. As an explanation, we note that more than 76 percent of the food retail stores in West Virginia are composed of convenience stores and small box retailers so that households are more likely to patronize these types of stores. Phillips and Rodriguez (2020) highlighted the issue of food swamps and diabetes-related hospitalization and found a positive relationship between diabetes-related hospitalizations and a higher index of food swamp severity.

Second, we consistently found that rural cold spots have higher chronic disease rates, and at the same time, increasing household income for the lower-income groups in these cold spots results in a lowering of chronic disease rates. Thus, improving access to healthy food providers might work in rural cold spots. Therefore, policies such as the healthy food financing that offer food retailers subsidies to locate in underserved areas should be encouraged in rural cold spots. Nonetheless, these policies should be accompanied by some means-tested benefits in order to boost the demand for nutritious food by low-income households. Such a policy recommendation is supported by other researchers who currently lobby for increases in SNAP benefits (Allcott et al., 2019). Our findings that income increases among low-income households reduce rates 
of chronic diseases shows that given the opportunity, these households would improve their healthy habits.

\subsection{Limitations}

Despite significant contributions in methodology and implications, a number of limitations should be acknowledged. First, when examining the food environment in West Virginia, we did not separate between healthy stores and non-healthy stores. Figure 2.1 in our study demonstrates how stores that sell food in West Virginia are largely dominated by convenience stores. Without a detailed data sets on how people shop at the census tract level, it is almost impossible to capture the effect of a healthy store on the health conditions of different demographics in a rural state such as West Virginia. This issue also did not allow us to study whether the differences in obesity and diabetes rates between hot and cold spots and their comparable non-hot and non-cold spots census tracts are driven by demand tastes or by the supply (as we suggest in this study). Second, future studies should utilize spatially measured data for diabetes and obesity rates collected at the census tract level. Finally, the Center for Disease Control (CDC) includes individuals who have diabetes and severe obesity within the group of those who are at a higher risk of being severely affected by COVID-19 ${ }^{11}$. Due to the lack of data for the years 2016 and 2017 and given this recent global health issue, this research did not include this variable - a new avenue of research for further studies would be to understand how place effects that impact food intakes are associated with this novel pandemic.

\footnotetext{
${ }^{11}$ https://www.cdc.gov/coronavirus/2019-ncov/need-extra-precautions/ groups-at-higher-risk.html\#severe-obesity
} 
2.7 Figures and Tables

Figure 2.1: Food Retail Distribution in West Virginia.

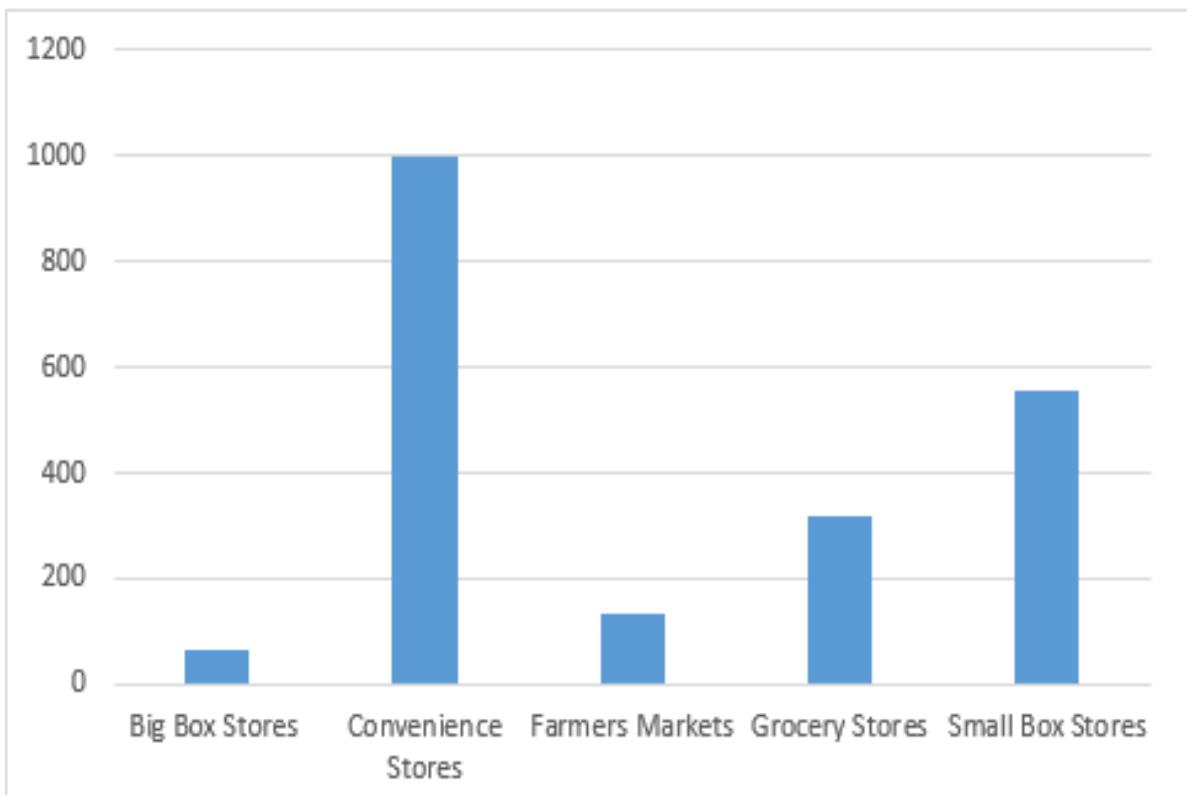


Figure 2.2: Hot Spots and Cold Spots of Food Providers in West Virginia.

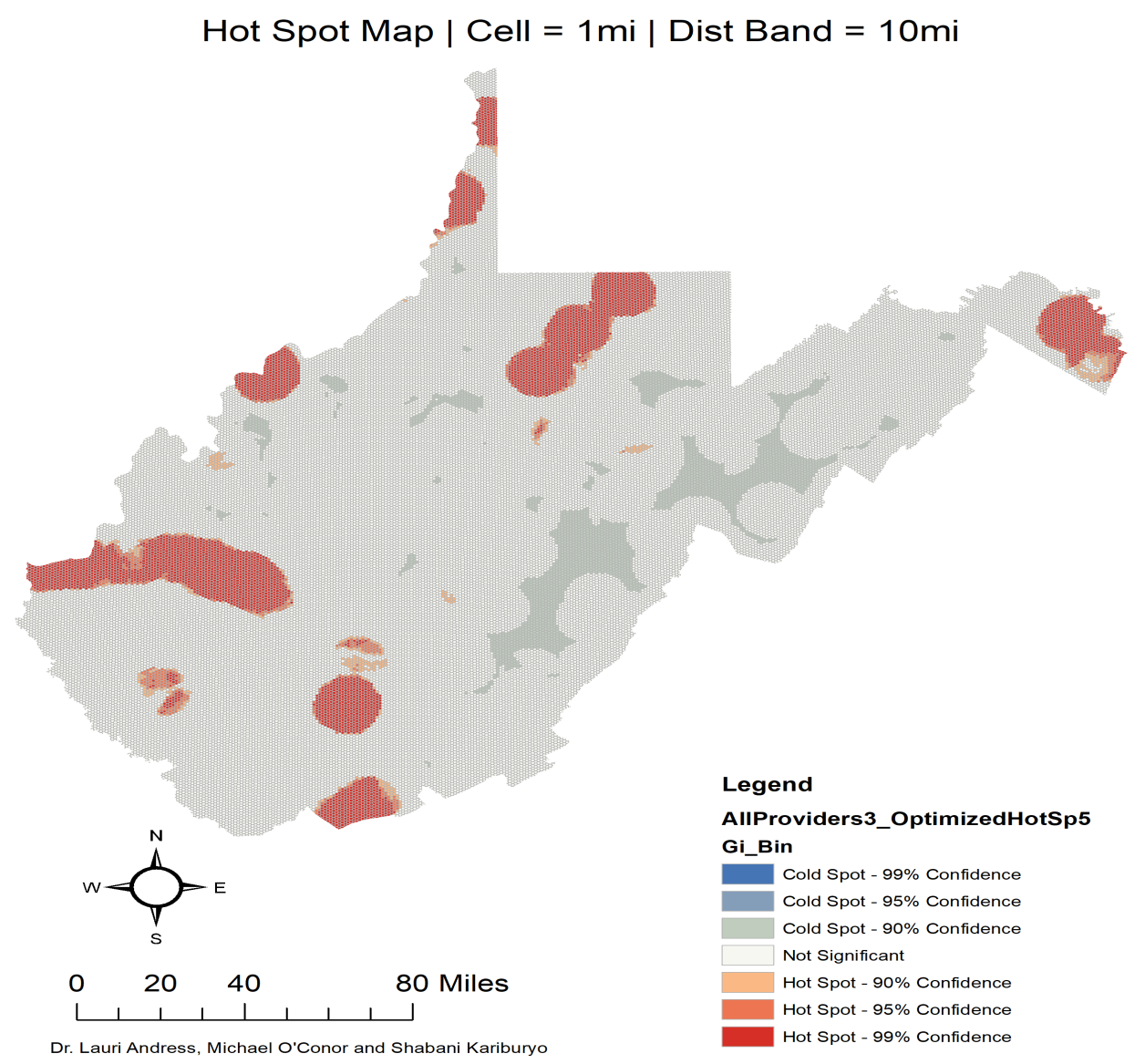


Figure 2.3: Food Retail Distribution and Poverty Rates.

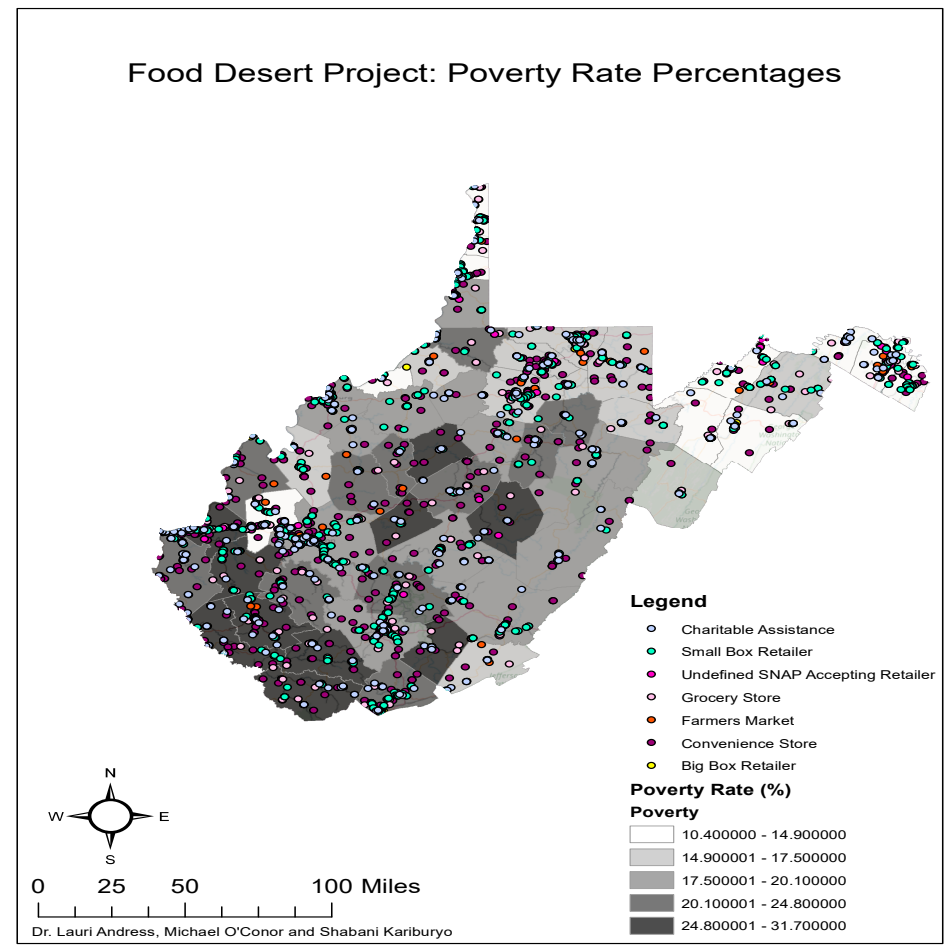


Figure 2.4: Heat Map of Food Providers in West Virginia.

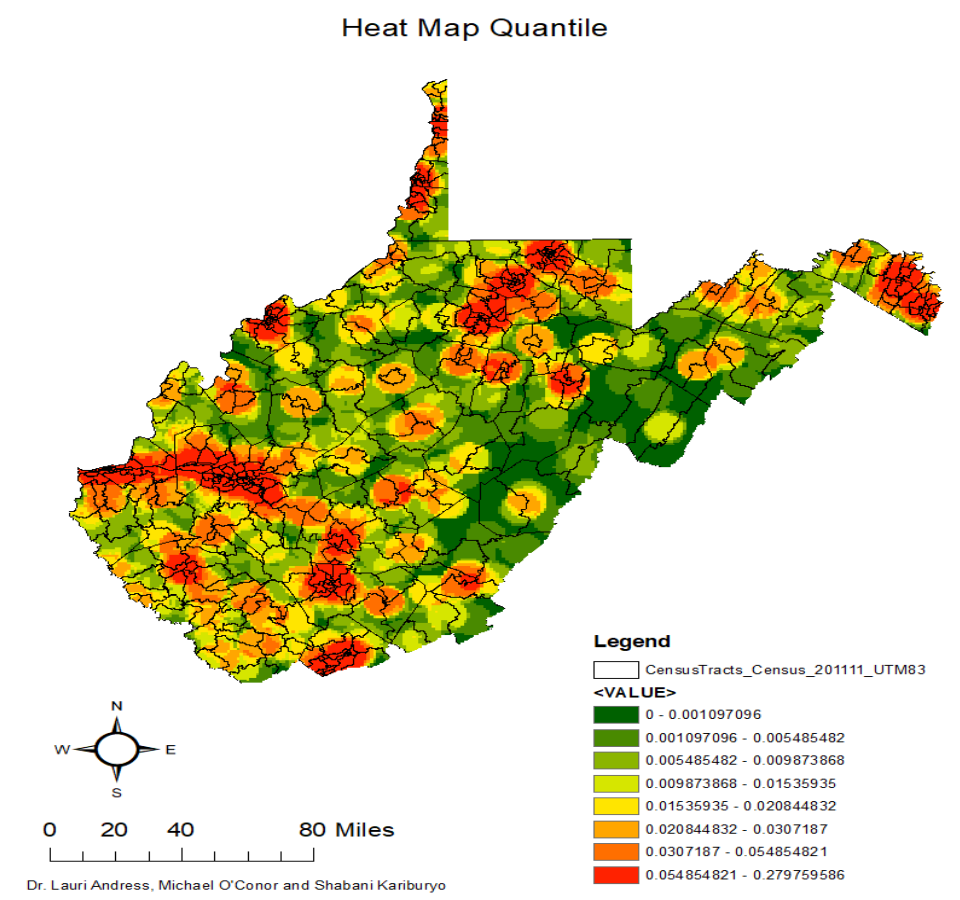


Table 2.1: Variable Descriptive Statistics for 2016 Data ( $t$-test in parentheses).

\begin{tabular}{|c|c|c|c|c|c|c|}
\hline & Hotspot (Mean) & Non-Hotspot (Mean) & Difference & Coldspot (Mean) & Non-Coldspot (Mean) & Difference \\
\hline Urban & 0.6333 & 0.2149 & \multirow{12}{*}{$\begin{array}{l}-0.4183 \\
(-10.0961) \\
0.0508 \\
(4.9497) \\
0.0019 \\
(1.0623) \\
-0.0074 \\
(-2.3575) \\
0.0345 \\
(4.2315) \\
-13.078 .47 \\
(-7.2750) \\
150 \\
(-1.2173) \\
-0.0566 \\
(-10.8271) \\
-0.0385 \\
(-5.6177) \\
-0.0056 \\
(-1.4515) \\
0.02075 \\
(6.2027) \\
0.1397 \\
(3.3315)\end{array}$} & 0.3043 & 0.4821 & \multirow{12}{*}{$\begin{array}{l}0.1777 \\
(3.1104) \\
-0.0289 \\
(-2.1837) \\
0.0029 \\
1.2688 \\
0.0052 \\
(1.2973) \\
-0.0032 \\
(-0.3095) \\
7134.2 \\
(3.0039) \\
130 \\
(0.8650) \\
0.0328 \\
4.5403) \\
0.0321 \\
(3.6340) \\
0.0074 \\
(1.5002) \\
-0.0193 \\
(-4.4825) \\
-0.1273 \\
(-2.3857)\end{array}$} \\
\hline Family Households (Proportion) & 0.6149 & 0.6657 & & 0.6608 & 0.6319 & \\
\hline Unemployment (Proportion) & 0.0404 & 0.4235 & & 0.0388 & 0.0418 & \\
\hline Health Insurance coverage (Proportion) & 0.9040 & 0.8965 & & 0.8965 & 0.9017 & \\
\hline Food stamp/Snap Benefits (Proportion) & 0.1588 & 0.1934 & & 0.1767 & 0.1735 & \\
\hline Mean Household Income (in 2016 dollars) & 72467.74 & 59389.27 & & 60906.92 & 68041.22 & \\
\hline Vehicle available & 2350 & 2200 & & 2180 & 2310 & \\
\hline Bachelor degree (Proportion) & 0.1428 & 0.0861 & & 0.0911 & 0.1240 & \\
\hline Non-White (Proportion) & 0.0757 & 0.0372 & & 0.0327 & 0.0648 & \\
\hline Age $<19$ (Proportion) & 0.1173 & 0.1116 & & 0.1088 & 0.1162 & \\
\hline Age $>59$ (Proportion) & 0.1694 & 0.1901 & & 0.19425 & 0.1749 & \\
\hline Community Health Services (Count) & 0.2481 & 0.3878 & & 0.4130 & 0.2857 & \\
\hline
\end{tabular}


Table 2.2: Covariate Balancing.

\begin{tabular}{|c|c|c|c|c|c|c|}
\hline & $\begin{array}{l}\text { Treatment Mean } \\
\text { (Hot Spots) }\end{array}$ & $\begin{array}{l}\text { Control Mean } \\
\text { (Before Weighting) }\end{array}$ & $\begin{array}{l}\text { Control Mean } \\
\text { (After Weighting) }\end{array}$ & $\begin{array}{l}\text { Treatment Mean } \\
\text { (Cold Spots) }\end{array}$ & $\begin{array}{l}\text { Control Mean } \\
\text { (Before Weighting) }\end{array}$ & $\begin{array}{l}\text { Control Mean } \\
\text { (After Weighting) }\end{array}$ \\
\hline \multirow{3}{*}{$\begin{array}{l}\text { Proportion of Family Household } \\
\text { Proportion of Unemployment } \\
\text { Proportion of Health Insurance } \\
\text { Coverage }\end{array}$} & 0.6149 & 0.6658 & 0.615 & 0.6609 & 0.6319 & 0.6609 \\
\hline & 0.0404 & 0.0423 & 0.0404 & 0.0388 & 0.0418 & 0.0388 \\
\hline & 0.9041 & 0.8966 & 0.9041 & 0.8965 & 0.9018 & 0.8965 \\
\hline $\begin{array}{c}\text { Proportion Food stamp/ } \\
\text { Snap benefits }\end{array}$ & 0.1588 & 0.1934 & 0.1588 & 0.1768 & 0.1735 & 0.11768 \\
\hline $\begin{array}{l}\text { Mean Household Income } \\
\text { Vehicle available }\end{array}$ & $\begin{array}{l}72468 \\
2351.6\end{array}$ & $\begin{array}{l}59389 \\
2350.3\end{array}$ & $\begin{array}{l}72468 \\
2351.6\end{array}$ & $\begin{array}{l}60907 \\
2182.3\end{array}$ & $\begin{array}{l}68041 \\
2311.2\end{array}$ & $\begin{array}{l}60907 \\
2182.3\end{array}$ \\
\hline Proportion of Bachelor degree & $\begin{array}{l}2351.0 \\
0.1428\end{array}$ & 0.08616 & 0.1428 & 0.0911 & 0.124 & $\begin{array}{l}2182.3 \\
0.09118\end{array}$ \\
\hline Proportion of Non-White & 0.0757 & 0.0372 & 0.07576 & 0.0327 & 0.06484 & 0.03272 \\
\hline Proportion of age $<19$ & 0.1173 & 0.1116 & 0.1173 & 0.1088 & 0.1162 & 0.1088 \\
\hline Proportion of age $>59$ & 0.1694 & 0.1902 & 0.1694 & 0.1942 & 0.1749 & 0.1942 \\
\hline Community Health Services & 0.2481 & 0.3879 & 0.2482 & 0.413 & 0.2857 & 0.413 \\
\hline
\end{tabular}


Table 2.3: The Impacts of Hot and Cold Spots on Diabetes Rates.

\begin{tabular}{|c|c|c|c|c|}
\hline Diabetes & $\begin{array}{c}\text { (1) } \\
\text { Unbalanced }\end{array}$ & $\begin{array}{c}(2) \\
\text { Balanced }\end{array}$ & $\begin{array}{c}\text { (3) } \\
\text { Unbalanced }\end{array}$ & $\begin{array}{c}(4) \\
\text { Balanced }\end{array}$ \\
\hline Treatment: Hot Spots & $\begin{array}{c}-0.0041^{* * *} \\
(0.000)\end{array}$ & $\begin{array}{c}-0.0054^{* * *} \\
(0.045)\end{array}$ & & \\
\hline Treatment: Cold Spots & & & $\begin{array}{l}0.0052 * * * \\
(0.009)\end{array}$ & $\begin{array}{c}0.0057 * * * \\
(0.002)\end{array}$ \\
\hline Constant & $\begin{array}{l}0.2236 \text { *** } \\
\quad(0.000)\end{array}$ & $\begin{array}{c}0.1354^{* * *} \\
(0.000)\end{array}$ & $\begin{array}{l}0.2190 * * * \\
(0.000)\end{array}$ & $\begin{array}{c}0.1388 * * * \\
(0.000)\end{array}$ \\
\hline Observation & 484 & 484 & 484 & 484 \\
\hline R-squared & 0.3150 & 0.0216 & 0.2411 & 0.0322 \\
\hline
\end{tabular}

Table 2.4: The Impacts of Hot and Cold Spots on Obesity Rates.

\begin{tabular}{|c|c|c|c|c|}
\hline Obesity & $\begin{array}{c}(1) \\
\text { Unbalanced }\end{array}$ & $\begin{array}{c}(2) \\
\text { Balanced }\end{array}$ & $\begin{array}{c}(3) \\
\text { Unbalanced }\end{array}$ & $\begin{array}{c}(4) \\
\text { Balanced }\end{array}$ \\
\hline Treatment: Hot Spots & $\begin{array}{c}-0.0128 * * * \\
(0.000)\end{array}$ & $\begin{array}{c}-0.0188 * * * \\
(0.000)\end{array}$ & & \\
\hline Treatment: Cold Spots & & & $\begin{array}{c}0.0052 * * * \\
(0.009)\end{array}$ & $\begin{array}{c}0.0137 * * * \\
(0.002)\end{array}$ \\
\hline Constant & $\begin{array}{l}0.3639 * * * \\
\quad(0.000)\end{array}$ & $\begin{array}{c}0.3533 * * * \\
(0.000)\end{array}$ & $\begin{array}{l}0.2190 * * * \\
(0.000)\end{array}$ & $\begin{array}{c}0.3483 * * * \\
(0.000)\end{array}$ \\
\hline Observation & 484 & 484 & 484 & 484 \\
\hline R-squared & 0.3500 & 0.1162 & 0.3453 & 0.0660 \\
\hline
\end{tabular}


Table 2.5: The Impacts of Urban and Rural Hot (and Cold) Spots on Obesity (2016).

\begin{tabular}{|c|c|c|c|c|}
\hline Obesity & (1) & $(2)$ & (3) & (4) \\
\hline Treatment: Urban Hot Spots & $\begin{array}{c}-0.0278^{* * * *} \\
(0.004)\end{array}$ & & & \\
\hline Treatment: Rural Hot Spots & & $\begin{array}{c}0.0185 * * \\
(0.077)\end{array}$ & & \\
\hline Treatment: Urban Cold Spots & & & $\begin{array}{l}0.0034 \\
(0.542)\end{array}$ & \\
\hline Treatment: Rural Cold Spots & & & & $\begin{array}{l}0.0273 * * * \\
(0.000)\end{array}$ \\
\hline $\begin{array}{l}\operatorname{Ln}(\text { Income }) \\
\text { 25th percentile }\end{array}$ & $\begin{array}{l}-0.0105 \\
(0.275)\end{array}$ & $\begin{array}{c}0.0022 \\
(0.700)\end{array}$ & $\begin{array}{c}-0.0087 * * * \\
(0.0076)\end{array}$ & $\begin{array}{c}-0.0024 \\
(0.596)\end{array}$ \\
\hline 25 th percentile $*$ Treatment & $\begin{array}{c}0.0234 * * \\
(0.035)\end{array}$ & $\begin{array}{c}-0.0159 \\
(0.216)\end{array}$ & $\begin{array}{l}0.0004 \\
(0.956)\end{array}$ & $\begin{array}{c}-0.0248 * * * \\
(0.006)\end{array}$ \\
\hline $\begin{array}{l}\text { Ln (Income) } \\
\text { 50th percentile }\end{array}$ & -0.0013 & $\begin{array}{l}-0.0123 \\
(0.105)\end{array}$ & $\begin{array}{c}-0.0100^{* * *} \\
(0.047)\end{array}$ & $\begin{array}{c}-0.0003^{* *} \\
(0.949)\end{array}$ \\
\hline 50 th percentile ${ }^{*}$ Treatment & $\begin{array}{c}-0.0146 \\
(0.227)\end{array}$ & $\begin{array}{c}-0.0295 * * \\
(0.022)\end{array}$ & $\begin{array}{c}-0.0010 \\
(0.886)\end{array}$ & $\begin{array}{c}-0.0244 * * * \\
(0.008)\end{array}$ \\
\hline $\begin{array}{l}\text { Ln (Income) } \\
\text { 75th percentile }\end{array}$ & $\begin{array}{l}-0.0122 \\
(0.189)\end{array}$ & $\begin{array}{l}-0.0023 \\
(0.662)\end{array}$ & $\begin{array}{c}-0.0267^{* * * *} \\
(0.000)\end{array}$ & $\begin{array}{c}-0.0065^{* * *} \\
(0.230)\end{array}$ \\
\hline 75th percentile ${ }^{*}$ Treatment & $\begin{array}{l}0.0159 \\
(0.129)\end{array}$ & $\begin{array}{c}-0.0193 * \\
(0.086)\end{array}$ & $\begin{array}{c}0.0240 * * * \\
(0.002)\end{array}$ & $\begin{array}{c}-0.0144 \\
(0.113)\end{array}$ \\
\hline Proportion of AFHM & $\begin{array}{c}0.1680 * * * \\
(0.000)\end{array}$ & $\begin{array}{c}0.02011 * * * \\
(0.000)\end{array}$ & $\begin{array}{c}0.1126 * * * \\
(0.001)\end{array}$ & $\begin{array}{c}0.0085 * * * \\
(0.001)\end{array}$ \\
\hline Constant & $\begin{array}{c}0.3489 * * * \\
(0.000)\end{array}$ & $\begin{array}{c}0.3326 * * * \\
(0.000)\end{array}$ & $\begin{array}{c}0.3549 * * * \\
(0.000)\end{array}$ & $\begin{array}{c}0.3455 * * * \\
(0.000)\end{array}$ \\
\hline Observation & 484 & 484 & 484 & 484 \\
\hline R-squared & 0.2184 & 0.1676 & 0.1346 & 0.1802 \\
\hline
\end{tabular}

Statistical significance: "*" 10 percent, "**" 5 percent, "***" 1 percent. $p$-value in parentheses. 
Table 2.6: The Impacts of Urban and Rural Hot (and Cold) Spots on Diabetes (2016).

\begin{tabular}{|c|c|c|c|c|}
\hline Diabetes & (1) & $(2)$ & (3) & (4) \\
\hline Treatment: Urban Hot Spots & $\begin{array}{c}-0.0084 \\
(0.095)\end{array}$ & & & \\
\hline Treatment: Rural Hot Spots & & $\begin{array}{l}0.0156 * * * \\
(0.005)\end{array}$ & & \\
\hline Treatment: Urban Cold Spots & & & $\begin{array}{c}-0.0061 \\
(0.089)\end{array}$ & \\
\hline Treatment: Rural Cold Spots & & & & $\begin{array}{c}0.01246 \text { *** } \\
(0.001)\end{array}$ \\
\hline $\begin{array}{l}\operatorname{Ln}(\text { Income }) \\
\text { 25th percentile }\end{array}$ & $\begin{array}{c}-0.0024 \\
(0.629)\end{array}$ & $\begin{array}{l}0.0020 \\
(0.581)\end{array}$ & $\begin{array}{c}-0.0087 * * * \\
(0.001)\end{array}$ & $-0.0042 *$ \\
\hline 25 th percentile ${ }^{*}$ Treatment & $\begin{array}{l}0.0043 \\
(0.515)\end{array}$ & $\begin{array}{l}-0.0142 \\
(0.054)\end{array}$ & $\begin{array}{r}0.0062 \\
(0.249)\end{array}$ & $\begin{array}{c}-0.0081 \\
(0.104)\end{array}$ \\
\hline $\begin{array}{l}\text { Ln (Income) } \\
\text { 50th percentile }\end{array}$ & $\begin{array}{c}-0.0086 \\
(0.097)\end{array}$ & $\begin{array}{l}-0.0029 \\
(0.427)\end{array}$ & $\begin{array}{c}-0.0105^{* * *} \\
(0.000)\end{array}$ & $\begin{array}{c}-0.0052 * * \\
(0.012)\end{array}$ \\
\hline 50 th percentile ${ }^{*}$ Treatment & $\begin{array}{l}0.0097 \\
(0.144)\end{array}$ & $\begin{array}{c}-0.0122 \\
(0.081)\end{array}$ & $\begin{array}{l}0.0053 \\
(0.223)\end{array}$ & $\begin{array}{c}-0.0116 * * \\
(0.014)\end{array}$ \\
\hline $\begin{array}{l}\text { Ln (Income) } \\
\text { 75th percentile }\end{array}$ & $\begin{array}{c}-0.0164^{* * *} \\
(0.003)\end{array}$ & $\begin{array}{c}-0.0078 * * \\
(0.042)\end{array}$ & $\begin{array}{c}-0.0207 * * * \\
(0.000)\end{array}$ & $\begin{array}{c}-0.0094 * * * \\
(0.001)\end{array}$ \\
\hline 75 th percentile $*$ Treatment & $\begin{array}{c}0.0135 * \\
(0.054)\end{array}$ & $\begin{array}{c}-0.0183 * * \\
(0.010)\end{array}$ & $\begin{array}{c}0.0224^{* * *} \\
(0.000)\end{array}$ & $\begin{array}{l}-0.0054 \\
(0.226)\end{array}$ \\
\hline Proportion of AFHM & $\begin{array}{c}0.0675 * * * \\
(0.007)\end{array}$ & $\begin{array}{c}0.0575 * * \\
(0.011)\end{array}$ & $\begin{array}{l}0.0555 * * * \\
(0.001)\end{array}$ & $\begin{array}{c}0.0465 * * * \\
(0.002)\end{array}$ \\
\hline Constant & $\begin{array}{c}0.1386 * * * \\
(0.000)\end{array}$ & $\begin{array}{c}0.1331 \text { *** } \\
(0.000)\end{array}$ & $\begin{array}{l}0.1455^{* * *} \\
(0.000)\end{array}$ & $\begin{array}{l}0.1404 * * * \\
(0.000)\end{array}$ \\
\hline Observation & & & & \\
\hline R-squared & 0.1066 & 0.1047 & 0.1798 & 0.1963 \\
\hline
\end{tabular}

Statistical significance: "*" 10 percent, "**" 5 percent, "***" 1 percent. $p$-value in parentheses. 
Table 2.7: Propensity Score Weighting Results for Diabetes.

\begin{tabular}{|c|c|c|c|c|}
\hline Diabetes & (1) & $(2)$ & $(3)$ & $(4)$ \\
\hline Treatment: Urban Hot Spots & $\begin{array}{c}-0.0116^{* * *} \\
(0.001)\end{array}$ & & & \\
\hline Treatment: Rural Hot Spots & & $\begin{array}{c}0.0127 * * \\
(0.015)\end{array}$ & & \\
\hline Treatment: Urban Cold Spots & & & $\begin{array}{l}0.0151 \\
(0.143)\end{array}$ & \\
\hline Treatment: Rural Cold Spots & & & & $\begin{array}{c}0.0293 \text { *** } \\
(0.008)\end{array}$ \\
\hline $\begin{array}{l}\operatorname{Ln}(\text { Income }) \\
25 \text { th percentile }\end{array}$ & $\begin{array}{c}-0.0069 * * * \\
(0.040)\end{array}$ & $\begin{array}{c}-0.0017 \\
(0.511)\end{array}$ & $\begin{array}{c}-0.0131 \\
(0.332)\end{array}$ & $\begin{array}{c}-0.0101 \\
(0.500)\end{array}$ \\
\hline 25 th percentile $*$ Treatment & $\begin{array}{c}0.0096 * \\
(0.064)\end{array}$ & $\begin{array}{l}-0.0099 \\
(0.173)\end{array}$ & $\begin{array}{l}0.0039 \\
(0.783)\end{array}$ & $\begin{array}{c}-0.0041 \\
(0.793)\end{array}$ \\
\hline $\begin{array}{l}\text { Ln (Income) } \\
\text { 50th percentile }\end{array}$ & $\begin{array}{l}-0.0116 \\
(0.002)\end{array}$ & $\begin{array}{c}0.0055 * \\
(0.075)\end{array}$ & $\begin{array}{l}-0.0016 \\
(0.892)\end{array}$ & $\begin{array}{l}-0.0025 \\
(0.847)\end{array}$ \\
\hline 50th percentile $*$ Treatment & $\begin{array}{c}-0.0131^{* *} \\
(0.015)\end{array}$ & $\begin{array}{l}-0.0109 \\
(0.120)\end{array}$ & $\begin{array}{c}-0.0047 \\
(0.696)\end{array}$ & $\begin{array}{l}-0.0218 \\
(0.008)\end{array}$ \\
\hline $\begin{array}{l}\text { Ln (Income) } \\
\text { 75th percentile }\end{array}$ & $\begin{array}{c}-0.0200 * * * \\
(0.000)\end{array}$ & $\begin{array}{c}-0.0114 * * * \\
(0.000)\end{array}$ & $\begin{array}{l}-0.0218 * \\
(0.057)\end{array}$ & $\begin{array}{l}-0.0180 \\
(0.134)\end{array}$ \\
\hline 75 th percentile $*$ Treatment & $\begin{array}{l}0.0159 * * * \\
(0.004)\end{array}$ & $\begin{array}{c}-0.0152 * * \\
(0.027)\end{array}$ & $\begin{array}{c}0.0261 * * * \\
(0.027)\end{array}$ & $\begin{array}{c}-0.0046 \\
(0.699)\end{array}$ \\
\hline Proportion of AFHM & $\begin{array}{l}0.0588 * * * \\
(0.003)\end{array}$ & $\begin{array}{c}0.0540 * * * \\
(0.001)\end{array}$ & $\begin{array}{l}0.2583 * * * \\
(0.000)\end{array}$ & $\begin{array}{l}0.1702 \text { *** } \\
(0.001)\end{array}$ \\
\hline Constant & $\begin{array}{l}0.1440 * * * \\
(0.000)\end{array}$ & $\begin{array}{c}0.1380 * * * \\
(0.000)\end{array}$ & $\begin{array}{l}0.1187 * * * \\
(0.000)\end{array}$ & $\begin{array}{l}0.1167 * * * \\
(0.000)\end{array}$ \\
\hline Observation & & 484 & 484 & 484 \\
\hline R-squared & 0.1440 & 0.1645 & 0.2881 & 0.3104 \\
\hline
\end{tabular}

Statistical significance: "*" 10 percent, "**" 5 percent, "***" 1 percent. $p$-value in parentheses. 
Table 2.8: Propensity Score Weighting Results for Obesity.

\begin{tabular}{|c|c|c|c|c|}
\hline Obesity & (1) & $(2)$ & (3) & (4) \\
\hline Treatment: Urban Hot Spots & $\begin{array}{c}-0.0332 * * * \\
(0.000)\end{array}$ & & & \\
\hline Treatment: Rural Hot Spots & & $\begin{array}{l}0.0117 \\
(0.310)\end{array}$ & & \\
\hline Treatment: Urban Cold Spots & & & $\begin{array}{l}0.0409 * * * \\
(0.000)\end{array}$ & \\
\hline Treatment: Rural Cold Spots & & & & $\begin{array}{l}0.0523 * * * \\
(0.000)\end{array}$ \\
\hline $\begin{array}{l}\text { Ln(Income) } \\
\text { 25th percentile }\end{array}$ & $\begin{array}{c}-0.0132 * * \\
(0.027)\end{array}$ & $\begin{array}{l}-0.0015 \\
(0.738)\end{array}$ & $\begin{array}{l}-0.0052 \\
(0.653)\end{array}$ & $\begin{array}{c}-0.0014 \\
(0.914)\end{array}$ \\
\hline 25 th percentile ${ }^{*}$ Treatment & $\begin{array}{c}0.0290 * * * \\
(0.001)\end{array}$ & $\begin{array}{c}-0.0112 \\
(0.435)\end{array}$ & $\begin{array}{l}0.0088 \\
(0.532)\end{array}$ & $\begin{array}{c}-0.0249 \\
(0.137)\end{array}$ \\
\hline $\begin{array}{l}\text { Ln (Income) } \\
\text { 50th percentile }\end{array}$ & $\begin{array}{c}-0.0077 \\
(0.248)\end{array}$ & $\begin{array}{l}0.0059 \\
(0.301)\end{array}$ & $\begin{array}{l}-0.0059 \\
(0.522)\end{array}$ & $\begin{array}{l}-0.0131 \\
(0.172)\end{array}$ \\
\hline 50 th percentile ${ }^{*}$ Treatment & $\begin{array}{c}-0.0249 * * * \\
(0.009)\end{array}$ & $\begin{array}{l}-0.0219 \\
(0.108)\end{array}$ & $\begin{array}{c}-0.0212 \\
(0.072)\end{array}$ & $\begin{array}{l}-0.0376 \\
(0.007)\end{array}$ \\
\hline $\begin{array}{l}\text { Ln (Income) } \\
\text { 75th percentile }\end{array}$ & $\begin{array}{c}-0.0174 * * \\
(0.015)\end{array}$ & $\begin{array}{c}-0.0081 * * * \\
(0.136)\end{array}$ & $\begin{array}{c}-0.0165 * \\
(0.053)\end{array}$ & $\begin{array}{l}-0.0099 \\
(0.194)\end{array}$ \\
\hline 75 th percentile $*$ Treatment & $\begin{array}{c}0.0221^{* * *} \\
(0.013)\end{array}$ & $\begin{array}{c}-0.0138 \\
(0.272)\end{array}$ & $\begin{array}{l}0.0186 \\
(0.100)\end{array}$ & $\begin{array}{c}-0.0047 \\
(0.712)\end{array}$ \\
\hline Proportion of AFHM & $\begin{array}{c}0.1233^{* * *} * \\
(0.003)\end{array}$ & $\begin{array}{c}0.1548 * * * \\
(0.001)\end{array}$ & $\begin{array}{c}0.4877^{* * *} \\
(0.000)\end{array}$ & $\begin{array}{c}0.3388 * * * \\
(0.000)\end{array}$ \\
\hline Constant & $\begin{array}{c}0.3561 * * * \\
(0.000)\end{array}$ & $\begin{array}{l}0.3411 * * * \\
(0.000)\end{array}$ & $\begin{array}{l}0.3069 * * * \\
(0.000)\end{array}$ & $\begin{array}{c}0.3033 * * * \\
(0.000)\end{array}$ \\
\hline Observation & 484 & 484 & 484 & 484 \\
\hline R-squared & 0.2311 & 0.1649 & 0.3931 & 0.4253 \\
\hline
\end{tabular}

Statistical significance: "*" 10 percent, "**" 5 percent, "***" 1 percent. $p$-value in parentheses. 
Table 2.9: Kernel Matching Results for Diabetes.

\begin{tabular}{|c|c|c|c|c|}
\hline Diabetes & (1) & $(2)$ & (3) & (4) \\
\hline Treatment: Urban Hot Spots & $\begin{array}{c}-0.0098 * * * \\
(0.008)\end{array}$ & & & \\
\hline Treatment: Rural Hot Spots & & $\begin{array}{c}0.0129 * * \\
(0.021)\end{array}$ & & \\
\hline Treatment: Urban Cold Spots & & & $\begin{array}{l}0.0069 \\
(0.404)\end{array}$ & \\
\hline Treatment: Rural Cold Spots & & & & $\begin{array}{c}0.0146 * * * \\
(0.001)\end{array}$ \\
\hline $\begin{array}{l}\mathrm{Ln}(\text { Income }) \\
\text { 25th percentile }\end{array}$ & $\begin{array}{l}-0.0049 \\
(0.123)\end{array}$ & $\begin{array}{c}-0.0007 \\
(0.794)\end{array}$ & $\begin{array}{c}-0.0053 * \\
(0.093)\end{array}$ & $\begin{array}{l}0.0006 \\
(0.870)\end{array}$ \\
\hline 25 th percentile ${ }^{*}$ Treatment & $\begin{array}{l}0.0068 \\
(0.203)\end{array}$ & $\begin{array}{l}-0.0111 \\
(0.122)\end{array}$ & $\begin{array}{l}0.0032 \\
(0.745)\end{array}$ & $\begin{array}{c}-0.0136 * * \\
(0.029)\end{array}$ \\
\hline $\begin{array}{l}\text { Ln (Income) } \\
\text { 50th percentile }\end{array}$ & $\begin{array}{c}-0.0112 * * * \\
(0.000)\end{array}$ & $\begin{array}{c}-0.0058 * * \\
(0.016)\end{array}$ & $\begin{array}{c}-0.0124 * * * \\
(0.000)\end{array}$ & $\begin{array}{c}-0.0068 * \\
(0.093)\end{array}$ \\
\hline 50th percentile ${ }^{*}$ Treatment & $\begin{array}{c}-0.0123^{* *} \\
(0.014)\end{array}$ & $\begin{array}{l}-0.0088 \\
(0.202)\end{array}$ & $\begin{array}{l}0.0072 \\
(0.499)\end{array}$ & $\begin{array}{c}-0.0105 * \\
(0.098)\end{array}$ \\
\hline $\begin{array}{l}\text { Ln (Income) } \\
\text { 75th percentile }\end{array}$ & $\begin{array}{c}-0.0124 * * * \\
(0.000)\end{array}$ & $\begin{array}{c}-0.0057 * * \\
(0.019)\end{array}$ & $\begin{array}{c}-0.0192 * * * \\
(0.000)\end{array}$ & $\begin{array}{c}-0.0080 * \\
(0.076)\end{array}$ \\
\hline 75th percentile ${ }^{*}$ Treatment & $\begin{array}{c}0.0095^{* *} \\
(0.038)\end{array}$ & $\begin{array}{c}-0.0194 * * * \\
(0.003)\end{array}$ & $\begin{array}{c}0.0203 * \\
(0.057)\end{array}$ & $\begin{array}{l}-0.0084 \\
(0.492)\end{array}$ \\
\hline Proportion of AFHM & $\begin{array}{l}0.0737 * * * \\
(0.000)\end{array}$ & $\begin{array}{c}0.0728^{* * * *} \\
(0.001)\end{array}$ & $\begin{array}{l}0.0255 \\
(0.252)\end{array}$ & $\begin{array}{l}0.0136 \\
(0.530)\end{array}$ \\
\hline Constant & $\begin{array}{l}0.1398^{* * * *} \\
(0.000)\end{array}$ & $\begin{array}{c}0.1344 * * * \\
(0.000)\end{array}$ & $\begin{array}{c}0.1473 \text { *** } \\
(0.000)\end{array}$ & $\begin{array}{c}0.1412 \text { *** } \\
(0.000)\end{array}$ \\
\hline $\mathrm{Ol}$ & & & & \\
\hline R-squared & 0.0876 & 0.0911 & 0.1311 & 0.1651 \\
\hline
\end{tabular}

Statistical significance: "*" 10 percent, "**" 5 percent, "***" 1 percent. $p$-value in parentheses. 
Table 2.10: Kernel Matching Results for Obesity.

\begin{tabular}{|c|c|c|c|c|}
\hline Obesity & (1) & $(2)$ & $(3)$ & (4) \\
\hline Treatment: Urban Hot Spots & $\begin{array}{c}-0.0307 * * * \\
(0.000)\end{array}$ & & & \\
\hline Treatment: Rural Hot Spots & & $\begin{array}{c}0.0187 * * \\
(0.025)\end{array}$ & & \\
\hline Treatment: Urban Cold Spots & & & $\begin{array}{l}0.0069 \\
(0.619)\end{array}$ & \\
\hline Treatment: Rural Cold Spc & & & & $\begin{array}{c}0.0383 * * * \\
(0.000)\end{array}$ \\
\hline $\begin{array}{l}\operatorname{Ln}(\text { Income }) \\
\text { 25th percentile }\end{array}$ & $\begin{array}{c}-0.0131^{* * *} \\
(0.004)\end{array}$ & $\begin{array}{l}-0.0113 \\
(0.786)\end{array}$ & $\begin{array}{c}-0.0017 \\
(0.748)\end{array}$ & $\begin{array}{l}0.0179 \\
(0.003)\end{array}$ \\
\hline 25 th percentile $*$ Treatment & $\begin{array}{l}0.0272^{* * * *} \\
(0.000)\end{array}$ & $\begin{array}{l}-0.0135 \\
(0.206)\end{array}$ & $\begin{array}{l}0.0095 \\
(0.569)\end{array}$ & $\begin{array}{c}-0.0413 * * \\
(0.000)\end{array}$ \\
\hline $\begin{array}{l}\text { Ln (Income) } \\
\text { 50th percentile }\end{array}$ & $\begin{array}{l}-0.0008 \\
(0.827)\end{array}$ & $\begin{array}{c}-0.0058 * * \\
(0.016)\end{array}$ & $\begin{array}{l}-0.0042 \\
(0.450)\end{array}$ & $\begin{array}{c}0.0114 * \\
(0.078)\end{array}$ \\
\hline 50 th percentile $*$ Treatment & $\begin{array}{c}-0.0149 * * \\
(0.039)\end{array}$ & $\begin{array}{l}-0.0122 \\
(0.001)\end{array}$ & $\begin{array}{l}0.0067 \\
(0.708)\end{array}$ & $\begin{array}{c}-0.0363 * * * \\
(0.098)\end{array}$ \\
\hline $\begin{array}{l}\text { Ln (Income) } \\
\text { 75th percentile }\end{array}$ & $\begin{array}{c}-0.0185^{* * *} \\
(0.000)\end{array}$ & $\begin{array}{c}-0.0080 * * \\
(0.029)\end{array}$ & $\begin{array}{c}-0.0175 * * * \\
(0.032)\end{array}$ & $\begin{array}{l}-0.0048 \\
(0.498)\end{array}$ \\
\hline 75th percentile $*$ Treatment & $\begin{array}{c}0.0234 * * * \\
(0.000)\end{array}$ & $\begin{array}{c}-0.0165 * \\
(0.092)\end{array}$ & $\begin{array}{l}0.0140 \\
(0.431)\end{array}$ & $\begin{array}{l}-0.0280 \\
(0.152)\end{array}$ \\
\hline Proportion of AFHM & $\begin{array}{c}0.127 * * * \\
(0.000)\end{array}$ & $\begin{array}{c}0.1518 * * * \\
(0.000)\end{array}$ & $\begin{array}{c}0.0720 * \\
(0.055)\end{array}$ & $\begin{array}{l}0.0386 \\
(0.530)\end{array}$ \\
\hline Constant & $\begin{array}{l}0.3524 * * * \\
(0.000)\end{array}$ & $\begin{array}{c}0.3339 * * * \\
(0.000)\end{array}$ & $\begin{array}{c}0.3527 * * * \\
(0.000)\end{array}$ & $\begin{array}{c}0.3387 * * * \\
(0.000)\end{array}$ \\
\hline $\mathrm{O}$ & & & & 484 \\
\hline R-squared & 0.2154 & 0.1649 & 0.0611 & 0.1859 \\
\hline
\end{tabular}

Statistical significance: "*" 10 percent, "**" 5 percent, "***" 1 percent. $p$-value in parentheses. 
Table 2.11: The Impacts of Urban and Rural Hot (and Cold) Spots on Diabetes (2017).

\begin{tabular}{|c|c|c|c|c|}
\hline Diabetes & (1) & $(2)$ & $(3)$ & (4) \\
\hline Treatment: Urban Hot Spots & $\begin{array}{c}-0.0087^{* *} \\
(0.050)\end{array}$ & & & \\
\hline Treatment: Rural Hot Spots & & $\begin{array}{c}0.0137 * * \\
(0.018)\end{array}$ & & \\
\hline Treatment: Urban Cold Spots & & & $\begin{array}{c}-0.0069 * * \\
(0.031)\end{array}$ & \\
\hline Treatment: Rural Cold Spots & & & & $\begin{array}{l}0.0127^{* * *} \\
(0.001)\end{array}$ \\
\hline $\begin{array}{l}\operatorname{Ln}(\text { Income }) \\
\text { 25th percentile }\end{array}$ & $\begin{array}{l}0.0008 \\
(0.848)\end{array}$ & $\begin{array}{l}0.0009 \\
(0.782)\end{array}$ & $\begin{array}{c}-0.0076 * * * \\
(0.002)\end{array}$ & $\begin{array}{l}-0.0030 \\
(0.143)\end{array}$ \\
\hline 25 th percentile $*$ Treatment & $\begin{array}{l}0.0001 \\
(0.977)\end{array}$ & $\begin{array}{l}-0.0025 \\
(0.735)\end{array}$ & $\begin{array}{l}0.0043 \\
(0.519)\end{array}$ & $\begin{array}{c}-0.0098 * \\
(0.058)\end{array}$ \\
\hline $\begin{array}{l}\text { Ln (Income) } \\
\text { 50th percentile }\end{array}$ & $\begin{array}{c}-0.0086 * \\
(0.085)\end{array}$ & $\begin{array}{l}-0.0033 \\
(0.445)\end{array}$ & $\begin{array}{c}-0.0108 * * * \\
(0.000)\end{array}$ & $\begin{array}{c}-0.0055 * \\
(0.009)\end{array}$ \\
\hline 50th percentile $*$ Treatment & $\begin{array}{c}-0.0144 \\
(0.025)\end{array}$ & $\begin{array}{l}-0.0102 \\
(0.164)\end{array}$ & $\begin{array}{c}0.0083 * * \\
(0.030)\end{array}$ & $\begin{array}{c}-0.0092 \\
(0.087)\end{array}$ \\
\hline $\begin{array}{l}\text { Ln (Income) } \\
\text { 75th percentile }\end{array}$ & $\begin{array}{c}-0.0179 * * * \\
(0.001)\end{array}$ & $\begin{array}{c}-0.0086 * \\
(0.052)\end{array}$ & $\begin{array}{c}-0.0162 * * * \\
(0.000)\end{array}$ & $\begin{array}{c}-0.0094 * * * \\
(0.001)\end{array}$ \\
\hline 75th percentile $*$ Treatment & $\begin{array}{c}0.0170 * * * \\
(0.013)\end{array}$ & $\begin{array}{c}-0.0164 * * \\
(0.026)\end{array}$ & $\begin{array}{l}0.0204 * * * \\
(0.000)\end{array}$ & $\begin{array}{l}-0.0054 \\
(0.348)\end{array}$ \\
\hline Proportion of AFHM & $\begin{array}{l}0.0840 \text { *** } \\
(0.001)\end{array}$ & $\begin{array}{l}0.0753 * * * \\
(0.001)\end{array}$ & $\begin{array}{c}0.0580 * * * \\
(0.000)\end{array}$ & $\begin{array}{l}0.0478^{* * *} \\
(0.001)\end{array}$ \\
\hline Constant & $\begin{array}{c}0.1371 * * * \\
(0.000)\end{array}$ & $\begin{array}{l}0.1327 * * * \\
(0.000)\end{array}$ & $\begin{array}{c}0.1454 \text { *** } \\
(0.000)\end{array}$ & $\begin{array}{c}0.1402 \text { *** } \\
(0.000)\end{array}$ \\
\hline Observation & 484 & 484 & 484 & 484 \\
\hline R-squared & 0.1462 & 0.1255 & 0.1646 & 0.1905 \\
\hline
\end{tabular}

Statistical significance: "*" 10 percent, "**" 5 percent, "***" 1 percent. $p$-value in parentheses. 
Table 2.12: The Impacts of Urban and Rural Hot (and Cold) Spots on Obesity (2017).

\begin{tabular}{|c|c|c|c|c|}
\hline Obesity & (1) & $(2)$ & $(3)$ & $(4)$ \\
\hline Treatment: Urban Hot Spots & $\begin{array}{c}-0.0260 * * * \\
(0.001)\end{array}$ & & & \\
\hline Treatment: Rural Hot Spots & & $\begin{array}{l}0.0212^{* *} \\
(0.049)\end{array}$ & & \\
\hline Treatment: Urban Cold Spots & & & $\begin{array}{l}-0.0004 \\
(0.946)\end{array}$ & \\
\hline Treatment: Rural Cold Spots & & & & $\begin{array}{c}0.0284 * * * \\
(0.001)\end{array}$ \\
\hline $\begin{array}{l}\operatorname{Ln}(\text { Income }) \\
\text { 25th percentile }\end{array}$ & $\begin{array}{l}0.0005 \\
(0.948)\end{array}$ & $\begin{array}{l}0.0036 \\
(0.519)\end{array}$ & $\begin{array}{c}-0.0090 * \\
(0.084)\end{array}$ & $\begin{array}{l}-0.0032 \\
(0.485)\end{array}$ \\
\hline 25 th percentile ${ }^{*}$ Treatment & $\begin{array}{l}0.0100 \\
(0.302)\end{array}$ & $\begin{array}{l}-0.0100 \\
(0.458)\end{array}$ & $\begin{array}{l}0.0029 \\
(0.742)\end{array}$ & $\begin{array}{c}-0.0297 * * * \\
(0.002)\end{array}$ \\
\hline $\begin{array}{l}\text { Ln (Income) } \\
\text { 50th percentile }\end{array}$ & $\begin{array}{l}-0.0044 \\
(0.652)\end{array}$ & $\begin{array}{c}-0.0163 * * \\
(0.043)\end{array}$ & $-0.0107 * *$ & $\begin{array}{c}0.00008 \\
(0.985)\end{array}$ \\
\hline 50th percentile ${ }^{*}$ Treatment & $\begin{array}{l}-0.0139 \\
(0.222)\end{array}$ & $\begin{array}{c}-0.0343 * * \\
(0.010)\end{array}$ & $\begin{array}{l}0.0034 \\
(0.639)\end{array}$ & $\begin{array}{c}-0.0232^{* *} \\
(0.018)\end{array}$ \\
\hline $\begin{array}{l}\text { Ln (Income) } \\
\text { 75th percentile }\end{array}$ & $\begin{array}{c}-0.0117 \\
(0.174)\end{array}$ & $\begin{array}{l}-0.0024 \\
(0.671)\end{array}$ & $\begin{array}{c}-0.0190 * * * \\
(0.002)\end{array}$ & $\begin{array}{c}-0.0092 * \\
(0.089)\end{array}$ \\
\hline 75th percentile*Treatment & $\begin{array}{c}0.0186 * \\
(0.059)\end{array}$ & $\begin{array}{c}-0.0234^{* *} \\
(0.045)\end{array}$ & $\begin{array}{c}0.0240 * * * \\
(0.003)\end{array}$ & $\begin{array}{l}-0.0049 \\
(0.642)\end{array}$ \\
\hline Proportion of AFHM & $\begin{array}{c}0.1650 * * * \\
(0.000)\end{array}$ & $\begin{array}{c}0.2053 * * * \\
(0.000)\end{array}$ & $\begin{array}{c}0.1215^{* * * *} \\
(0.000)\end{array}$ & $\begin{array}{c}0.0478 * * * \\
(0.001)\end{array}$ \\
\hline Constant & $\begin{array}{c}0.3453 * * * \\
(0.000)\end{array}$ & $\begin{array}{c}0.3314 * * * \\
(0.000)\end{array}$ & $\begin{array}{c}0.3544 * * * \\
(0.000)\end{array}$ & $\begin{array}{c}0.3449 * * * \\
(0.000)\end{array}$ \\
\hline Observation & 484 & 484 & 484 & 484 \\
\hline R-squared & 0.2515 & 0.2131 & 0.1266 & 0.2003 \\
\hline
\end{tabular}




\section{Chapter 3}

\section{Colonial Heritage, Traditional, and}

\section{Chinese Foreign Direct}

\section{Investments in Africa: A Dynamic Spatial Interdependence Analysis}

\subsection{Introduction}

The African continent is one of the fastest urbanizing regions globally, and the foreign direct investments (FDIs) tremendously assist African governments in enduring the rapid demographic transition associated with urbanization. However, since the early 2000s, countries from the Organization for Economic Co-operation and Development $(\mathrm{OECD})$ which are considered as traditional investors in Africa, have been divesting while China is swiftly extending FDIs and other types of finances in Africa. Chinese and western countries FDIs both seek resources and profit. However, unlike western countries' FDIs, Chinese FDIs have a higher share of investments in African countries that have weaker governance (Chen et al., 2018). Furthermore, traditional 
investors are more reluctant to send FDIs to countries that are financially tied to China (Dreher et al., 2021b, 2019; Donou-Adonsou and Lim, 2018). Given the complex foreign policies in most parent countries and the intense competition between some countries globally, an interesting question is how these FDIs interact on the African continent.

Another important aspect about FDI to Africa is their links to historical colonial ties (Lemi et al., 2021; Madden, 2019). The sources of this link can be traced back to the nature of institutions imposed by colonizers that still persist in Africa today (Acemoglu et al., 2001). For example, Lundan and Jones (2001) show that the intra-Commonwealth trade and investment is favored by the use of the English language, coupled with similarities in institutional structures, legal systems, and business practices between the United Kingdom and other host countries within the Commonwealth. The long lasting effects of colonization is also reiterated by (Liou and RaoNicholson, 2017), who explain that factors such as languages and institutions from the colonizers are still dictating the way some developing countries conduct business. Further examining FDI in developing countries, Makino and Tsang (2011) point out that in prior research identification, the relational factors that have been historically created between the host and the parent countries' are often times overlooked, with most studies focusing on the traditional trade determinant's such as geographical distances and trade agreements.

In the FDI literature, studies have taken two approaches to study the factors that influence parent countries' decision of sending investments to host countries-a bilateral framework and a framework that includes third country effects (Blonigen et al., 2007; Baltagi et al., 2007). On the one hand, the bilateral frameworks aim at understanding horizontal FDI since in this case Multinational Enterprises (MNEs) are implemented in a host country for market access purposes to substitute for export flows (Blonigen et al., 2007; Baltagi et al., 2007). On the other hand, studies that have accounted for third country effects aim at understanding FDIs that have strategies like those of an export-platform investment, vertical or complex vertical FDIs (Blonigen 
et al., 2007; Nwaogu and Ryan, 2014; Poelhekke and Van Der Ploeg, 2013; Siddiqui and Iqbal, 2018; Lemi et al., 2021) ${ }^{1}$. Blonigen et al. (2007) and Baltagi et al. (2007) propose using spatial econometrics models to account for the spatial interdependence of these FDIs that arise from the third countries effects. However, in most of these spatial FDI studies, the spatial linkages are defined by the geographical distances (Siddiqui and Iqbal, 2018), and the use of historical ties highlighted by (Makino and Tsang, 2011) to define these spatial linkages is still lacking in the literature.

To fill the void in this literature, this study explores the spatial interdependence of Chinese and traditional FDIs (American, Dutch and French) in Africa, while using a weight matrix that takes into consideration Africa's historical colonial ties. Unlike Nwaogu and Ryan (2014) that find that American FDIs in Africa are complex vertical while using a row normalized inverse distance weight matrix, our study finds that there are no interdependencies of American FDIs in Africa. Furthermore, we also find that an increase in Chinese FDIs in a country that share the same colonial ties with another African country reduces Chinese FDIs in that country. These results are different than those found in (Lemi et al., 2021) who use the row-normalized inverse distance weight matrix to find a relationship that is the other way around. It is worth mentioning that the row-normalized inverse distance weight matrix used in (Blonigen et al., 2007; Nwaogu and Ryan, 2014; Lemi et al., 2021) has come under scrutiny as we will explain in the next section.

We also find that Dutch and French FDIs in Africa have vertical strategies i.e. French and Dutch MNEs send their affilates in Africa for factor cost purposes only; and Chinese FDIs in Africa serve as export platforms. The results hold even when we check for the sensitivity of our historical ties weight matrix, by using an inverse

\footnotetext{
${ }^{1}$ For example, export platform FDIs seek to serve third countries market by using their affiliate in a host country to export the goods. In the case of vertical FDIs, the parent country send its affiliates to a host country for factor cost purposes. In a more sophisticated case of vertical FDIs, MNEs send their affiliates in multiple host countries to exploit comparative advantages of various countries "complex vertical FDI".In all the last cases of FDIs motives, one can see that a simple two country framework might suffer from the omitted variables issue, since the only determinants of FDIs included are those of the host country included in the study (Blonigen et al., 2007; Baltagi et al., 2007).
} 
distance weight matrix that divide each row with the highest eigen value (Regelink and Elhorst, 2015; Siddiqui and Iqbal, 2018; Kelejian and Prucha, 2010), a method that correct for the misspecification of the row normalized weight matrix. ${ }^{2}$. Finally, we find that Chinese FDIs crowd out Dutch FDIs in African countries, and an increase in Chinese FDIs in an African country also reduce American FDIs in the whole continent.

To our knowledge, this is the first spatial FDI paper that takes into account space and time while studying the spatial interdependencies's of FDIs. Furthermore, we are also the first study that use a historical colonial ties weight matrix in lieu of the widely used distance based weight matrix. Finally, this study is the first of its kind that thoroughly analyze the differences in FDI motives between a non traditional investor (China), and traditional investors (US, the Netherlands, and France). The remainder of this paper is structured as follows. We begin by reviewing previous literature on Chinese and traditional investors finances in Africa, as well as other papers on the spatial interdependence of FDIs in Section 3.2. Section 3.3 presents the data and empirical methodology. In Section 3.4, we discuss the main results along with sensitivity analysis. Finally, Section 3.5 concludes this study and discusses how these FDIs strategies might be at work on the African continent while highlighting some limitations.

\subsection{Literature Review}

This paper draws from two strands of the literature. The first one relates to the difference between Chinese and traditional investors' finances in Africa. The second strand relates to the spatial interdependence of Foreign Direct Investments. While the two strands are relatively extensive, there is still another avenue of research within both that hasn't been explored, and this paper hopes to fill this void.

\footnotetext{
${ }^{2}$ In the sensitivity analysis, compared to the historical ties weight matrix, the Surrounding Market Potentials in the Chinese FDI models turn out not stastically significant. Furthermore, the spatially lagged Dutch FDI variable became not statistically significant. A part from these variables, our results are consistent with the sensitivity analysis results.
} 


\subsubsection{Chinese Finances in Africa}

Much of the existing literature on Chinese finances in Africa focus on development assistance finances and the impact that these finances have had on Africa's economic development (Dreher et al., 2021a, 2018, 2019, 2021b). For example, Dreher et al. (2018) discuss that much of the criticism from the western world that Chinese finances in Africa receive comes from the failure to distinguish between China's official development assistance and more commercially oriented sources and types of state financing, such as FDIs. Further studying Chinese finances and their impact on economic development in Africa, Dreher et al. (2021a) find that the local receipt of Chinese aid does improve local development outcomes - even though Chinese aid tends to favor the birth region of local political leaders, this study finds that Chinese aid is effective at promoting economic development.

There are also studies that have investigated whether Chinese finances support politically motivated and economically inefficient projects which tend to be avoided by western countries' development agencies. While studying the differences between Chinese and the Word Bank's projects in Africa, Dreher et al. (2019) find that Chinese finances in Africa bankrolls politically motivated and economically inefficient ("white elephant") projects, which tend to be avoided by western aid agencies, such as the World Bank (Dreher et al., 2021a). Furthermore, Dreher et al. (2021b) find that Chinese finances spur short-term economic growth, without necessarily impairing OECD countries' development aid effectiveness. Nonetheless, American aid tends to be more effective in countries that do not receive Chinese assistance. In this current study, we shift from Chinese concessional and non-concessional finances to another type of external financing, Foreign Direct Investments. We also analyze Chinese FDIs in comparison with FDIs from traditional African partners (OECD countries).

The literature on Chinese FDIs in Africa has been rather thin, and comparative studies on the effectiveness of this new source of investment compared to traditional OECD countries investors are lacking in the literature. Some of the studies that have treated this issue find that the factors that attract Chinese and traditional FDIs in 
Africa are different (Chen et al., 2018; Tuman and Shirali, 2017a). Chen et al. (2018) investigate Chinese FDIs in Africa as compared to western countries FDIs, with a simple question in mind, why is China investing in Africa? They find that Chinese FDIs, just like western countries' FDIs, are profit-driven. This study also finds that like western countries, Chinese FDIs in Africa is resource seeking. Key differences in these two sources of FDIs that these study highlights are found in the variables related to the quality of institutions. Unlike western FDIs, China has a higher share of investments in countries with weak governance. Furthermore, this study also finds that Chinese FDIs in Africa are not associated with the rule of law variable.

Tuman and Shirali (2017a) do reiterate the negative association between Chinese FDIs and institutional variables, but they also show that countries with diplomatic ties with Taiwan have a hard time attracting Chinese FDIs. Further studying Chinese and western FDIs in Africa, Donou-Adonsou and Lim (2018) find that Chinese FDIs increase income in Africa, but American and German FDI tend to improve African income even higher. Furthermore, this study finds that Chinese FDIs crowd out American FDIs in Africa, but not French FDIs. It is important to note that all these previous studies failed to incorporate spatial or locational components in their studies, which, as we are about to see in the next subsection, are important determinants of FDIs (Blonigen et al., 2007; Baltagi et al., 2007).

\subsubsection{Spatial Interdependence of FDI}

In the past decade, a number of studies have started recognizing the importance of incorporating spatial variables in FDI models (Blonigen et al., 2007; Baltagi et al., 2007; Blanco, 2012; Nwaogu and Ryan, 2014; Regelink and Elhorst, 2015; Lemi et al., 2021; Siddiqui and Iqbal, 2018; Poelhekke and Van Der Ploeg, 2013). The argument made by these studies is that previous bilateral FDI models failed to capture the third country effects created by the interdependence between FDI decisions to alternative host countries. Baltagi et al. (2007) and Blonigen et al. (2007) underline both the 
importance of adding spatial variables in FDI models since omitting them would create a bias in the estimator. In the spatial FDI literature, the spatial interdependence of MNE investments in competing receiving countries is tested by two variables: the Surrounding Market Potential (SMP) which are macroeconomic variables of neighboring markets ${ }^{3}$ and the spatially weighted FDI variable of neighboring countries. Table 3.1 highlights the different motives found in the literature.

There are spatial FDI studies that have specifically focused their attention on the African continent (Nwaogu and Ryan, 2014; Siddiqui and Iqbal, 2018; Lemi et al., 2021). The main differences between these papers are the variables representing the sources of FDI inflows to Africa used, the African regions included, and the normalization of the inverse distance weight matrix used in most of these studies. Nwaogu and Ryan (2014) use American FDIs to African, Latin, and Caribbean countries to study the spatial interdependence of these MNEs in the receiving countries. They find that spatial effects existed indeed, and that American FDIs to these countries are consistent with the complex FDI with specialization types. Few criticisms that this study usually receives: are their use of spatial autoregressive model (SAR) to capture this spatial interdependence, and the way they normalize their distance weight matrix.

Furthermore, Nwaogu and Ryan (2014); Blonigen et al. (2007); Lemi et al. (2021) normalize their distance weight matrix by row-normalizing the inverse distance, a method that has been shown to lead to a misspecification problem ${ }^{4}$ (Kelejian and Prucha, 2010; Regelink and Elhorst, 2015; Siddiqui and Iqbal, 2018). Kelejian and

\footnotetext{
${ }^{3}$ Usually captured by the GDP of the neighboring countries or the population size (Blonigen et al., 2007; Poelhekke and Van Der Ploeg, 2013).

${ }^{4}$ To illustrate this situation, consider for example, remote and central countries that may end up having the same impact, i.e. regardless of their relative location. One example to illustrate could be countries such as Tunisia and Gabon in our study. In this type of normalization, the distance between Tunisia and its neighbors is represented by d (suppose also that there are no other neighbors and that the distance to the two neighbors is the same), while the distance of Gabon to its neighbors is a multiple of $d$. Despite this difference in distance, the entries in the inverse distance matrix describing the spatial arrangement of Tunisia and Gabon to its neighbors will be $1 / 2$ and sum to 1 in both cases, provided that the spatial weights matrix is row-normalized. Further note that exactly for this reason the inverse distance matrix is generally not row-normalized when calculating the market potential variable(Blonigen et al., 2007; Poelhekke and Van Der Ploeg, 2013).
} 
Prucha (2010); Siddiqui and Iqbal (2018); Regelink and Elhorst (2015) propose a normalization procedure where each element of $\mathrm{W}$ is divided by its largest eigenvalue. In the row normalized matrix, the highest eigenvalue is one whereas in the Kelejian and Prucha (2010); Regelink and Elhorst (2015) normalization process, the proportions between the elements of the spatial weights matrix remain unchanged.

Thus, existing studies focus only on the spatial interdependence of Chinese and American FDIs on the African continent-which shows how thin this literature is because Africa receives FDIs from other countries as well. Furthermore, even the closest study to ours Lemi et al. (2021) only look at the spatial determinants of Chinese and American FDIs in African countries and, as mentioned in the previous paragraphs, this study suffers from the issue of normalizing the inverse distance weight matrix. By studying the spatial interdependence of American, Chinese, Dutch, and French FDIs in Africa, while taking into account African countries colonial heritage, this study hopes to close the gaps in this literature.

\subsection{Empirical Methods and Data}

\subsubsection{Data}

We retrieve FDI flows to 42 African countries from France, the Netherlands, the US, and China from the OECD and the China-Africa Research Initiative (CARI). FDI data from 2002 to 2013 are available for the four parent countries. In addition, we use the World Bank's database to collect several macroeconomic, institution, infrastructure, development, and governance indicators. Table 3.2 exhibits variables, their definition, units, and sources, along with the list of 42 African nations.

\subsubsection{Empirical Strategy}

We begin with a benchmark OLS model that represents the determinants of FDI flows in Africa. The literature has shown that market potential, natural resources, 
infrastructures, institutions and the level of protectionism of a country all affect FDI flows into African countries:

$$
\begin{aligned}
\ln (F D I)_{i t}= & \alpha+\beta_{1} \ln (F D I)_{i, t-1}+\beta_{2} \text { GDP } \text { growth }_{i, t-1}+\beta_{3} \ln (\text { pop })_{i, t}+\beta_{4} \ln (\text { open })_{i, t} \\
& +\beta_{5} \ln (N R E)_{i, t-1}+\beta_{6} \text { inflation }_{i, t}+\beta_{7} \text { infrastructure }_{i, t}+\beta_{8} \text { Pol climate }_{i, t} \\
& +\mu_{i}+\nu_{t}+\epsilon_{i, t}
\end{aligned}
$$

where, the dependent variable $\ln (F D I)_{i t}$ is the natural log of FDI flows between a parent country (e.g., China, France, the Netherlands and the US) and an African country $i$ in time $t . \alpha, \sigma_{i}$, and $\mu_{i t}$ are the constant term, the country effects, and the error term, respectively.

We include various determinants of FDI flows in the regression equation, which can be broadly categorized as economic, institutional and infrastructural variables (Asiedu, 2006; Hasnat, 2013; Lemi et al., 2021; Tuman and Shirali, 2017b). GDP growth $\left(G D P\right.$ growth $\left._{i, t-1}\right)$ and the population size $\left(\ln (p o p)_{i, t-1}\right)$ in the host country are used to proxy market potentials. A larger market size is likely to attract more FDIs to the recipient country. Another measure of market potential included in this study is inflation $\left(\right.$ inflation $\left._{i, t}\right)$. Because of the adverse impact that inflation has on sales, market oriented firms might avoid African host countries with higher inflation (Tuman and Shirali, 2017b). The natural log of trade openess $\left(\right.$ open $\left._{i, t-1}\right)$ is a proxy of African countries national protectionism, and capture the barriers that might be between the home and host countries (Regelink and Elhorst, 2015; Carr et al., 2001).

Government effectiveness index is used to proxy the political climate (Pol climate $_{i, t}$ ), and hence the quality of institutions in the African host countries. As noted earlier, traditional FDIs may favor countries with better institutions, while non-traditional FDIs can better adjust to adverse political conditions (Dreher et al., 2019; Tuman and Shirali, 2017a; Santiso, 2008; Chen et al., 2018). We use the electric power con- 
sumption per capita to proxy infrastructure. Countries with better infrastructure (infrastructure $_{i, t}$ ) are more likely to attract more FDIs due to the favorable business climate that these countries offer (Nwaogu and Ryan, 2014; Siddiqui and Iqbal, 2018). Finally, the natural resource rent variable $\left(\ln (N R E)_{i, t-1}\right)$ is included to understand resource seeking FDIs, and the lag of the natural $\log$ of FDI $\left(\ln (F D I)_{i, t-1}\right)$ has been added to address serial correlation. This variable helps us understand how FDI inflows adjust over time (Egger and Merlo, 2007).

One criticism of the model outlined in equation 3.1 is that it may fail to capture the interdependence of FDI decisions to alternative host countries (Blonigen et al., 2007). Several studies recognize the importance of incorporating spatial variables in FDI models (Blanco, 2012; Baltagi et al., 2007; Nwaogu and Ryan, 2014; Siddiqui and Iqbal, 2018; Regelink and Elhorst, 2015; Lemi et al., 2021; Blonigen et al., 2007). Furthermore, Baltagi et al. (2007), and Blonigen et al. (2007) note that adding spatial variables in FDI models can help mitigate the omitted variable bias as only controlling for the host country variables without accounting for neighboring country's factors might violate some OLS assumptions.

Following LeSage and Pace $(2009,2014)$ we consider the Spatial Durbin Model (SDM) framework that comprises a dynamic spatial panel with fixed effects:

$$
\begin{array}{r}
\ln (F D I)_{i t}=\gamma+\delta \ln (F D I)_{i t-1}+\rho \mathbf{W} \ln (F D I)_{i t}+\omega \mathbf{W} \ln (F D I)_{i t-1} \\
+\mathbf{X}^{\prime} \Gamma+\mathbf{W} \mathbf{X}^{\prime} \Theta+\mu_{i}+\nu_{t}+\epsilon_{i t}
\end{array}
$$

where, $\mathbf{W}$ is $n \times n$ spatial weight matrix that captures the connectedness between two pairs of African countries. For notation convenience, we use $\mathbf{X}$, a $k \times T$ matrix, to represent the $k$ vectors of determinants of FDI as presented in equation 3.1, and $\ln (F D I)_{i t-1}$ is included in these variables to understand how the previous year FDI inflows affect the current year inflows. Further, $\mathbf{W} \ln (F D I)_{i t}$ is the spatial lag of the dependent variable and $\mathbf{W X}^{\prime}$ spatially lagged variable of the independent variables, $\mathbf{W} \ln (F D I)_{i t-1}$ is included in these variables to understand how FDI spatially adjust 
over time. The coefficients $\rho$ is the spatial auto-regressive parameter. The vector $\Gamma$ and $\Theta$ are $k \times 1$ vector of unknown parameters. Following Elhorst (2014), we include fixed effect in the spatial model to capture time and country invariant unobservables.

Furthermore, Equation 3.2 is important in finding out traditional and Chinese FDIs motives in Africa, as shown in Table 3.1. Throughout this table, one can tell that the signs of the spatial lag $\left(\mathbf{W} \ln (F D I)_{i t}\right)^{5}$ and the surrounding market potential (SMPs) are dictating the motives of FDIs. In our case, we follow Poelhekke and Van Der Ploeg (2013) and Regelink and Elhorst (2015), and use the indirect effects of the GDP growth, (WGDP growth $_{i, t-1}$ ), and the natural log of the population size, $\left(W \ln (p o p)_{i, t-1}\right)$, as our SMPs. In other words, when $\rho=0$ and $\Theta=0$ for GDP growth and population size, we have the case of a horizontal FDI. When $\rho=-$ and $\Theta=+$, we have the case of an export platform FDI. Finally, when $\rho=-$ and $\Theta=0^{6}$ and $\rho=+$ and $\Theta=+/-$, we have the case of a purely vertical FDI and complex vertical with fragmentation.

Many countries in Africa define their commercial legal traditions according to their colonization history. Ganau (2017) and La Porta et al. (1999) argue that countries sharing the same legal origin may have strong linkages, and therefore, might have more notable spillover effects among themselves. A weight matrix that takes into consideration that African countries that share the same legal heritage -from their colonization history - may have higher commercial and trade linkages between them has never been used in the spatial FDI literature (Ganau, 2017; La Porta et al., 1999). Therefore, we investigate spatial spillovers via this weight matrix channel. In addition, the literature supports the idea that the current African socio-economic and institutional settings may be tied to their colonial links (Nunn, 2007). Moreover, empirical evidence suggests a robust relationship between economic development and African countries' colonial history (Grier, 1999; Acemoglu et al., 2001; Englebert,

\footnotetext{
${ }^{5}$ or in some cases $\left(\mathbf{W} \ln (F D I)_{i, t-1}\right)$ - the indirect effects of the spatially time lagged of the dependent variable.

${ }^{6}$ as shown in Table 3.1, in some cases involving resource FDI, we could have vertical FDIs that have $\rho=-$ and $\Theta=-$.
} 
2000).

Following such argument, we define a spatial weight matrix based on the African countries historical ties with European countries- England, France, Belgium, Portugal and Italy $^{7}$. Hence we define $\mathbf{W}$ as following:

$$
\mathbf{W}=\frac{\left(\frac{1}{d_{i j}^{g e o}} \times d_{i j}^{\text {legal }}\right)^{2}}{\sum_{j}\left(\frac{1}{d_{i j}^{g e o}} \times d_{i j}^{\text {legal }}\right)}
$$

where, $1 / d_{i j}^{g e o}$ is the inverse of the geographic distance between country $i$ and $j$ given as the distance in kilometers between the geographic centroids of the two African countries. $d_{i j}^{\text {legal }}$ is a dummy variable that is equal to 1 if countries $i$ and $j$ have the same colonial heritage, and it equals to 0 otherwise.

The inclusion of the spatial interlinkages allow us to disentangle the motives of FDIs. From the SDM framework of Equation 3.2, we can compute the direct, indirect, and total effects impacts that arise due to the changes in explanatory variables from one unit at a specific period. The interpretation of the direct and indirect effects includes the partial and cross-partial derivatives of the dependent variable with respect to the independent variables (Regelink and Elhorst, 2015; LeSage and Pace, 2009). We then use the intuition provided in Table 3.1 to interpret the motives of Chinese, American, French and Dutch FDIs in Africa.

Next, we examine whether Chinese FDIs crowd-in or crowd-out French, Dutch, and American FDI in Africa. To do this, we define the dependent variable as the natural log of FDI flows from US, France and the Netherlands and we augment Equation 3.2 with the lagged natural $\log$ of Chinese FDI flows $\ln (F D I)_{i t-1, \text { Chinese }}$ and its spatial lag $\mathbf{W} \ln (F D I)_{i t-1, \text { Chinese }}$ :

\footnotetext{
${ }^{7}$ for more information please see Table 3.3
} 


$$
\begin{array}{r}
\ln (F D I)_{i t, F r e n c h, \text { American,Dutch }}=\gamma+\delta \ln (F D I)_{i t-1}+\rho \mathbf{W} \ln (F D I)_{i t}+\omega \mathbf{W} \ln (F D I)_{i t-1} \\
+\zeta \ln (F D I)_{i t-1, \text { Chinese }}+\pi \mathbf{W} \ln (F D I)_{i t-1, \text { Chinese }}+\mathbf{X}^{\prime} \Gamma+\mathbf{W X}^{\prime} \Theta+\mu_{i}+\nu_{t}+\epsilon_{i t}
\end{array}
$$

The results that we find are then compared to Donou-Adonsou and Lim (2018) who investigate whether Chinese FDIs in Africa are crowding out investments from OECD countries without accounting for third countries' effects.

\subsection{Results}

\subsubsection{Distribution and Trends of Chinese, Dutch, French and American FDI Flows}

The geographic distribution of FDI flows to Africa is shown in Figure 3.3, which comprises four panels of percentile maps. These maps show extreme values, i.e., African countries in the top $1 \%$ and $10 \%$, as well as countries that are in the bottom $10 \%$ of FDI inflows during our study period. Overall, we can see that Nigeria received most of the Dutch FDIs during our study period, South Africa attracted most Chinese FDIs. Finally, Egypt had the most American and French FDIs.

Figure 3.1 shows an upward trend of Chinese FDI flows in Africa, whereas American and French FDIs in Africa seems to be fluctuating over the years. Our data shows that around 2009, Chinese flows started surpassing both American and French FDIs. Dutch FDI flows are still low compared to other FDIs, though their pattern also seems to fluctuate over the years, just like other traditional FDIs. Eom et al. (2017) show that throughout the years since 2000, the share of American FDI stock has been diminishing in Africa. Figure 3.2 corroborates these findings and shows that Chinese FDI stocks in Africa have been booming from $\$ 15$ billion in 2011 to almost $\$ 27$ billion in 2013. Another country growing FDI stocks in Africa is the Netherlands, 
from around $\$ 12$ billion in 2011 to more than $\$ 20$ billion in 2013. Figure 3.2 also shows that during the last year of our study sample, the United States and France had the most FDI stocks in Africa.

With Dutch and Chinese FDIs showing an increasing trend while American and French FDI showing signs of decreasing and stagnating throughout Africa, there is a justifiable reason to thoroughly analyze the motives of these FDIs (horizontal, export platform, vertical integration, or complex vertical). Moreover, the behavior of FDI observed in these figures lead us in investigating how these FDI flows interact on the African continent, do they increase or decrease each other on the continent? The next set of regressions aim to do that by answering these two questions with empirical evidence.

\subsubsection{The Spatial Interdependence of Chinese, Dutch, French and American FDI Flows}

We begin our estimation results with Table 3.5 which presents the baseline estimates of equation 3.1 and the cross-sectional dependency tests. The cross-sectional dependence test allows us to detect cross-country auto-correlation or cross-sectional dependence. We use Pesaran (2015) test of weak cross-sectional dependence of residuals and detect potential cross African countries dependence for French, Dutch, and Chinese FDIs, but not for American FDIs. This test is a preliminary diagnostic of whether spatial motives exist in subsequent regressions.

In Table 3.6, we consider 42 countries that are in the contiguous African continent and present the direct, indirect, and total impacts of FDIs from France, the Netherlands, the United States, and China. We use equation 3.2 and the historical colonial ties weight matrix to investigate whether FDIs are propagating in countries that share the same colonial roots. In this table, we analyze the different FDI motives explained in Table 3.1. In the following interpretations, we focus more on the spatial lag of the dependent variable, the surrounding market potential variables (SMPs), and other in- 
dependent variables that are important to our analysis such as, the natural resources independent variable.

In Table 3.6, column (1)-(3), we show estimates regarding French FDIs. In column (1), we find that previous year's French FDI flows positively affect the current flows of French FDI within African countries. The indirect effect of the natural log of last year's French FDI flows given in column (2) is not statistically significant. But, the spatial lag of the dependent variable is negative and statistically significant, suggesting that a 10 percent increase in French FDIs in countries that share the same colonial heritage, reduce about 3 percent of French FDI flows in another African country with the same colonial history. Furthermore, apart from the direct effect of the natural resource variable, and the proxy for the political climate, the government effectiveness, other determinants of FDI are not significant. However, the total effects of the previous year's natural log of French FDI flows are positive, which show that French FDI flows on the whole continent are dependent on previous years FDIs, highlighting the importance of accounting for the serial correlation of the FDI variable. This result signals that regional or continental efforts in attracting French FDIs can be effective in Africa.

Therefore, French FDIs in Africa are vertically integrated since French FDI flows in one African country are at the expense of other countries with the same colonial heritage. The indirect effects of the GDP growth and the natural log of the population size, our surrounding market potential variables are not significant. Blonigen et al. (2007) and Regelink and Elhorst (2015) explain that the vertical strategy takes place when the spatial lag variable is negative, and the surrounding market potential variables (the indirect effects of the GDP growth and population size in our case) ${ }^{8}$ are not significant ${ }^{9}$. Furthermore, Poelhekke and Van Der Ploeg (2013) also suggest that most vertical FDIs are mostly resource FDI, because refining is mainly done in

\footnotetext{
${ }^{8}$ Regelink and Elhorst (2015) advise using the indirect effects of the market potential variables, in our case, GDP growth and population size, as the surrounding market potential variables. In this study, they also interpret a negative spatial lag combined with insignificant SMPs as vertical FDIs.

${ }^{9}$ For more on the motives, see Table 3.1
} 
the home markets close to the final consumers, and hence the human capital in the host countries does not play a big role. For the French FDIs case, we also see that the direct effect of the natural resource variable is positive and statistically significant, which reinforces our point that French FDIs in Africa are a pure form of vertical FDI.

Table 3.6 column (4)-(6) show the results for Dutch FDIs. The direct effect shows that previous Dutch FDI flows in an African country bring more Dutch FDIs into this country. Furthermore, the spatial lag variable is negative and statistically significant. Nonetheless, the time lagged indirect effect of Dutch FDIs shows that a 10 percent increase in Dutch FDIs in countries that share the same colonial roots in the previous year, will increase Dutch FDI flows in an African country with the same colonial history by 2 percent. The different signs and statistically significant results of the indirect effect of the time lagged Dutch FDI variable and the spatially lagged dependent variable could be showing two strategies: a complex vertical with fragmentation or a vertically integrated FDI strategy (see Table 3.1).

On the one hand, one could assign the vertical strategy with integration to Dutch FDIs in Africa, since the indirect effects of the SMPs variables show a statistically insignificant GDP growth, and a negative and statistically significant natural log of the population size. The latter stems from the fact that most vertical FDIs are resource seeking, and the size of the population plays a non-significant role in attracting these types of FDIs, since most of natural resources are exported back to the home country to be treated next to the final consumer (Poelhekke and Van Der Ploeg, 2013).

On the other hand, the direct effect of the natural resource variable is negative and statistically significant, suggesting that Dutch FDIs in Africa are non-resource FDIs. While investigating the motives of Dutch FDIs, Poelhekke and Van Der Ploeg (2013) show the need to differentiate between Dutch's non-resource and resource FDIs ${ }^{10}$. While we do not have the disagregated data for both resource and non-resource Dutch FDIs, we conclude that the positive and statistically significant indirect effects of

\footnotetext{
${ }^{10}$ Poelhekke and Van Der Ploeg (2013) illustrate how Dutch non-resource and resource FDI have different motives, with non-resource FDIs being complex FDI with fragmentation, and resource FDIs being vertically integrated.
} 
the previous year Dutch FDIs $\left(\operatorname{Ln}(F D I)_{i, t-1}\right)$ coupled with the signs of the SMPs variables, and the negative statistically significant natural resource variable could be picking up the non-resource FDI strategies, which are indicative of complex vertical with fragmentation strategies (Poelhekke and Van Der Ploeg, 2013). The findings that the indirect and total effects of our infrastructure proxy, the electricity consumption, and our measure of trade openness are statistically significant strengthen the idea that Dutch FDIs could be a complex vertical with fragmentation since similar studies find these variables to be tied to this FDI motive (Poelhekke and Van Der Ploeg, 2013; Baltagi et al., 2007).

The subsequent regression in Table 3.6, column (7)-(9), analyze the spatial interdependence of American FDI flows in African countries through the channels of historical colonial ties. The spatially auto-correlated dependent variable is not significant, just as the Pesaran (2015) CD test suggested in Table 3.5. Despite the non-significance of our primary variable, the SDM model with the historical colonial ties weight matrix still presents some interesting facts. For example, we learn from this regression some of the main direct, indirect, and total effects of American FDI flows in African countries. For example, a 10 percent increase in the previous year American FDI in one African country would increase that country's American FDI flows by 3.7 percent during the following year. Furthermore, the total effect shows that an increase in the previous year American FDIs in an African country by ten percent would increase American FDIs on the whole continent by 3.9 percent. Other variables that show some significant spatial total effects are the level of openness of a country, natural resource endowment, GDP growth, the natural log of the population size $^{11}$, and a good political climate.

Table 3.6 columns (10) - (12) show the results with the natural log of Chinese FDIs as our dependent variable. The spatial lag of the dependent variable is negative

\footnotetext{
${ }^{11}$ Since the spatially lagged dependent variable of American FDI is not statistically significant, we cannot determine the motives of American FDIs. However, the negative and statistically significant total effects of the SMPs show some signs resource vertical FDI. Since these FDIs do not require good market potential variables, since they are only interested in natural resources.
} 
and statistically significant. An increase by ten percent in Chinese FDI flows in an African country will decrease Chinese FDI flows in another country that has the same colonial history by 0.9 percent. Contrary to Dutch and French FDIs, both the population size and the GDP growth are statistically significant and positive. These two variables' results and the negative spatial lag of Chinese FDI in Africa lead us to conclude that Chinese FDI in Africa have an export platform strategy when we use the historical colonial ties weight matrix. In this case, Chinese MNEs seek to produce products in Africa and export those rather than selling them in the host countries. These results make sense since most Chinese manufacturing companies in Ethiopia, for example, export a large chunk of their goods to a larger European market or other African countries (Tang, 2019). However, Tang (2019) also explain that Chinese manufacturers in Ethiopia also serve within this country. The total effect column also inform us that a ten percent increase in Chinese FDI flows in an African country leads to an increase of 3.8 percent in Chinese FDI flows through the rest of Africa. Apart from our SMP variables, other variables that attract Chinese FDI are the proxy for infrastructure and inflation. The latter highlights the argument that non-traditional FDIs tend to locate their FDIs in countries with poor macroeconomic conditions.

Overall, these results show that some of the criticisms that Chinese finances in Africa receive need to be revisited. These results highlight how traditional investors are simply different from non-traditional investors, at least in this case of China, France, American and the Netherlands. With export platform MNEs, China can create knowledge transfer since it has to work with local governments to gain from this type of investment. For the vertical Dutch and French FDIs, little is needed from the local government because the sole purpose of French and Dutch affiliates is to export back to the parent country. Nonetheless, the findings that Dutch FDIs have a complex vertical strategy and a vertical strategy with integration, highlight the shortcomings of using agreggated data, instead of using disagregated data. We use the same models that are augmented with the spatially lagged of Chinese FDI in 
the following subsection to understand whether Chinese FDIs crowd out/or crowd in French, Dutch, and American FDIs.

\subsubsection{Spatial Crowding In and Crowding Out of Chinese FDIs on Traditional FDIs}

We examine whether China-Africa's partnership altered preexisting relationships between Africa and its traditional partners, which is a highly debated point in development economics. For example, Dreher et al. (2021b) investigate whether Chinese finances in Africa undermine the effectiveness of development finances from western donors. Our empirical strategy estimates the spatial crowding in and crowding out of Chinese, French, Dutch, and American FDIs in Africa. We augment the previous spatial interdependence models of Dutch, French, and American FDI with the lagged natural log of Chinese FDI. By doing this, we want to understand how a percentage change in one source of FDI affects the inflows of the other source of FDI. Since this study compares Chinese and OECD countries' FDIs, we want to show how Chinese MNEs interact with the OECD countries' MNEs on the African continent.

Table 3.7 shows the results of our specifications that aim at understanding how the MNEs are interlinked in Africa. We start by investigating the spatial impact of Chinese FDIs on French FDI flows in columns (1)-(3). This specification controls for all the independent variables used in the previous spatial interdependence models and analyzes the direct, indirect, and total effects. The augmented spatial lagged of the natural $\log$ of Chinese FDI flows to Africa is not statistically significant. However, in columns (4)-(6), we find that an increase by 10 percent in the previous year' Chinese FDI flows in an African country causes it to lose about 0.2 percent of its Dutch FDIs.

Furthermore, in column (9), we see that a 10 percent increase in Chinese FDIs in an African country lead to a 5.8 percent decrease in American FDIs on the African continent. The results found in Table 3.7 are consistent with those of Dreher et al. (2021b), Annen and Knack (2019), and Donou-Adonsou and Lim (2018). However, 
in two of these studies, the main topic is on development assistance finances, and in Donou-Adonsou and Lim (2018), they perform a study without accounting for third country effects.

The results in the above table inform us that Chinese FDIs are crowding out American and Dutch FDIs on the African continent. In contrast, we do not see any statistically significant results on the French regression. We argue that French FDIs might be competing with Chinese FDIs on the African continent since one is solidifying its old links on the continent (as you can see in Figure 3.2, French FDI stocks are still high), and the other is just starting to cement his ties. DonouAdonsou and Lim (2018) also report the same findings that France and China are competing in Africa. Both Figure 3.1 and 3.2 highlight the fact that the United States is divesting, which probably explains why there is a crowding-out effect of Chinese FDI on American FDI on the whole continent. The negative relationship between Chinese and Dutch FDIs in African countries could be that the two countries are just after different factors, as illustrated by the different significance of the various non-spatial and spatial determinants. Overall, the use of the models that account for space and time turn out important since these results were found in the direct and total effects, which is a way of understanding whether African countries should focus on regional or continental-wide policies while studying ways of attracting traditional and non-traditional FDIs.

\subsubsection{Sensitivity Analysis}

As for sensitivity analysis, we present the spatial auto-regressive model (SAR $)^{12}$ to capture the spatial interdependence of various FDIs in African countries. SAR model is similar to SDM model given in equation 3.2, except it excludes the the spatial lag of the explanatory variables WX. Unlike the SDM model, the SAR model faces criticism that it only includes the spatially lagged FDI variables and does not include

\footnotetext{
${ }^{12} \ln (F D I)_{i t}=\gamma+\delta \ln (F D I)_{i t-1}+\rho \mathbf{W} \ln (F D I)_{i t}+\mathbf{X}^{\prime} \Gamma+\mu_{i}+\nu_{t}+\epsilon_{i t}$
} 
the spatial lag of the explanatory variables - which are very important in deciding the surrounding markets' potential variables (Siddiqui and Iqbal, 2018; Regelink and Elhorst, 2015). LeSage and Pace (2009) and LeSage and Pace (2014) argue that the Spatial Durbin Model (SDM) should be the preferred method of estimation as it allows for the possibility of directly including other explanatory variables.

Despite these criticisms, we present the SAR model as a sensitivity check in Table 3.8. Meanwhile, the log-likelihood, which assesses the goodness of the fit between the SDM and SAR, is also presented in Table 3.8. This test gives us a sense of reassurance in choosing between the SAR or the SDM when one believes that omitted variables in the model are spatially correlated, and these spatially correlated omitted variables are correlated with an included explanatory variable in the model. The LR tests in Table 3.8 suggests that in all the cases, including the spatially lagged independent variables in the SDM models of Table 3.6 improved the fit of our models. Furthermore, the statistically significant spatial lag of the natural log of US FDI in Table 3.8 does not align with the Pesaran (2015) CD test in Table 3.5. Therefore, it is concerning to us that the SAR model with the historical colonial ties weight matrix has this variable statistically significant.

We also assess to what extent our results change when we switch our weight matrix to the distance weight matrix used in (Siddiqui and Iqbal, 2018; Regelink and Elhorst, 2015). The elements of the matrix are the distances between countries. We use the capital city of each country as the center of the country to obtain these distances. The elements of the matrix are characterized as $1 / d_{i j}^{g e o}$, the inverse of the distances between each country. Finally, we normalize our weight matrix just as in Kelejian and Prucha (2010), Regelink and Elhorst (2015) and Siddiqui and Iqbal (2018) who normalize this distance-based weight matrix by dividing each element of $\mathrm{W}$ by its largest eigenvalue.

Table 3.9 shows the results that we obtained by using this distance weight matrix. Compared to the SDM with the historical colonial ties weight matrix, we only find that the spatial lag of the dependent variables for the natural log of French and Chinese 
FDI are negative and statistically significant, but this time, the spatial lag of the Dutch FDI is not significant. Similar to the results of the SDM with the institutional weight matrix, the spatial lag of the American FDI is not statistically significant. The one difference between Table 3.9 and 3.6 that strikes us the most is the nonsignificance of the two SMP variables, population size and GDP growth that had led us in concluding that Chinese MNEs in Africa have an export platform motive. In the case of the distance-based weight matrix, we find that Chinese FDIs have the same motive as French FDI, i.e., they all display vertical strategies in Africa. Nonetheless, even these results do make sense in some instances in Africa. Poelhekke and Van Der Ploeg (2013) suggest that most resource FDI are vertical. For example, China has Foreign Direct Investments in the cobalt industry in the Democratic Republic of Congo which have increased in production and in capacity over time (Gulley et al., 2019). As it is the case under vertical integration strategies, Chinese MNEs affiliates ship back most of the raw materials to the parent country, where most of the cobalt is refined (Poelhekke and Van Der Ploeg, 2013).

Compared to Lemi et al. (2021), our main results and the sensitivity analysis differ considerably. The main differences between this study and theirs is the weight matrix used. In our study, we use a weight matrix with colonial historical ties, and compares it to an inverse distance weight matrix (standardized by dividing each row element with the highest eigen value), and they standardize their weight matrix a la Nwaogu and Ryan (2014) and Blonigen et al. (2007), an approach as seen in Section 4.2, that leads to the misspecification of the models. Unlike Lemi et al. (2021) who compare only the interests of Chinese and American FDIs, we also include French and Dutch FDIs to this debate.

Their results are different from our main and sensitivity analysis results. For example, the spatial lag of the dependent variable is positive for the spatially lagged of Chinese FDIs variable in their case, and it turned out negative in ours, implicating that the motives explained in Lemi et al. (2021) are suffering from the misspecification issue that Regelink and Elhorst (2015), Siddiqui and Iqbal (2018) and Kelejian and 
Prucha (2010) noted when using the row-normalized inverse distance weight matrix. Furthermore, the results of the SMP variables are also misleading in (Lemi et al., 2021). They turned out not significant in the case of Chinese FDIs-something we also see in our sensitivity analysis results but not with the results from our main results. Their results for American FDIs also show that the spatial lag is not significant. It is important to note that Lemi et al. (2021), Nwaogu and Ryan (2014), Siddiqui and Iqbal (2018) fail to incorporate a cross sectional dependence test or even a test for spatial auto correlation in their study. The Pesaran (2015) CD test was very important in our case, because it helped us see whether FDIs were spatially interdependent in all of our 4 cases.

Overall, we learn from these sensitivity analyses that the SDM model is usually a better fit than the SAR model when studying the spatial interdependence of FDIs. Furthermore, the spatially lagged dependent variable of Dutch FDIs turned out not significant, and the SMPs variables became statistically not significant for the Chinese FDIs regression when we changed to the distance-based weight matrix. In conclusion, these results highlight the fact that our results might be sensitive to the different types of weight matrices. Nonetheless, the consistent findings of a non-significant spatial lag of American FDIs, and the negative signs on the spatial lags of Chinese, Dutch and French FDIs insinuate that the spatial processes in our main and sensitivity analysis results might be the same.

\subsection{Conclusion and Implications}

Existing studies on Chinese finances in Africa primarily focus on foreign aid and the non-spatial and spatial determinants of FDI (e.g., Dreher et al., 2021b, 2016; Donou-Adonsou and Lim, 2018; Chen et al., 2018; Lemi et al., 2021). However, since Africa has received FDI from various actors, we conduct a comparative study that accounts for the differences between non-traditional FDIs (China) and traditional FDIs in Africa. We contribute to this literature by studying the spatial motives of 
French, Dutch, American, and Chinese FDIs flows to African countries. In addition, Africa has many investments from other partners; we consider only these four to understand how South-South FDIs behave compared to North-South FDIs on the African continent.

Using a panel of 42 African countries from 2002-2013 and an institutional-based weight matrix that is tied to the historical colonial ties of African countries, we find that, on the one hand, European countries such as France and the Netherlands' investments in Africa have vertical strategies. French and Dutch MNEs evaluate potential African countries' destinations to find the one that is the lowest cost provider of the activity it wishes to outsource or conduct. On the other hand, this study finds that Chinese MNEs in Africa serve as an export platform for servicing third-country markets. However, it is worth noting that when we switch from the institutional weight matrix to a distance-based weight matrix in our sensitivity analysis, Chinese MNEs strategies change to a vertical strategy. These results are found by assessing the significance level and signs of the spatial lag of the dependent variable and the surrounding market potential (SMP) variables, in our case, the population size and GDP growth.

Furthermore, we investigate the spatial crowd in and crowd out between Chinese and French, American and Dutch FDIs while using the same historical colonial ties matrix. We find that Chinese FDIs and some of the traditional investors on the African continent, American and Dutch FDIs, adversely impact each other. An increase in Chinese FDI leads to a decrease in American and Dutch FDIs. Meanwhile, French and Chinese FDIs in Africa are not crowding out each other, but we conclude that they are currently competing because one is solidifying its old ties on the continent, and the other is building its reputation. This is illustrated by the boom in economic forums that are organized by France and China in Africa in the past decade. ${ }^{13}$ Our results complement those of the foreign aid literature (Dreher et al.,

\footnotetext{
${ }^{13}$ https://chinaafricaproject.com/2019/10/30/france-africa-business-forum-opens-amidquestions-over-how-the-french-can-compete-against-the-chinese/
} 
2016, 2021b; Annen and Knack, 2019) in understanding the differences and the interaction between Chinese and OECD ' countries finances in Africa.

To put our results into perspective, we give few examples from news articles and official global trade websites of how our empirical findings might be at work on the African continent. Consider the cut flower market in Kenya; about 50 percent of exported flowers from Kenya are sold through Dutch Auctions. While these two countries are ranked in the top four of cut flowers global wholesales, we can see how Kenya is largely reliant on the Dutch Vertically integrated FDIs in this sector to conduct its business. The literature on Chinese dealings in Africa also highlights the going out strategies that the Chinese government has implemented to find raw materials and fuel its ambitious economic development plans (Foster, 2009). One way that the Chinese vertically integrated FDI strategy might be at work is via the favorable loans that the Democratic Republic of $\mathrm{Congo}^{14}$ receives in exchange for the copper and cobalt mineral development rights that they give to Chinese MNEs for example (Gulley et al., 2019). With the findings found with the historical colonial ties weight matrix that Chinese MNEs serve as export platforms in Africa, the example of Tecno Mobile phones success is the most prevalent in the news. ${ }^{15}$. This brand which is mainly unpopular in China can be found in many African capital cities such as Lagos, Nairobi, and Addis-Ababa. Transsion's assembly factory in Addis Ababa, Ethiopia, is one of the export platforms of Tecno Mobile in Africa.

This study showed that Chinese MNEs in Africa are not different from Traditional MNEs in Africa to some extent, as all of these countries are resource seeking and are vertically integrated in Africa. However, Chinese MNEs differ from traditional MNEs in their export platform strategies that we do not see from OECD countries or traditional FDIs. In this case, we see how China is using its experience as a country that is just shifting from a developing country to a developed economic power to implement brand such as Tecno Mobile that has a motto that defines most of Chinese

\footnotetext{
${ }^{14}$ https://www.newyorker.com/magazine/2021/05/31/the-dark-side-of-congos-cobalt-rush

${ }^{15}$ https://www.cnn.com/2018/10/10/tech/tecno-phones-africa/index.html
} 
MNEs in Africa: "Think Global, Act Local." With the findings that Chinese FDIs are spatially interdependent in Africa, future studies should focus on understanding the different spatial channels through which the recent Zambia's Chinese loan default repayments are manifesting themselves (Brautigama and Ackerb, 2021), which is a hot topic at the moment. We should be aware that the results could be sensitive to the weight matrix used in all these studies.

We would also like to explain some of the limitations of our study. First, we use Chinese FDI data from the John Hopkins University China Africa Research Initiative (CARI). CARI has combined this data from China's Statistical Yearbooks and Statistical Bulletins of China's Outward Foreign Direct Investment of different years. Other studies have used data from the China Global investment tracker (Lemi et al., 2021). In the literature, there is a lack of consensus as to which data to use. We go with the aggregated data from CARI due to the high-quality data collection and field research reputation attributed to this center. While we believe in the accuracy of our results, we believe that data that is broken down at the sector or industry level could be more telling. Finally, as mentioned earlier, we could mainly use the year 2002 up until 2013 since it was the only year available for French and Dutch FDI. We believe that adding recent years to our study could have added more talking points related to the policy implication of our research. 


\subsection{Figures and Tables}

Figure 3.1: Trends of French, Dutch, American, and Chinese FDI (in millions dollars)

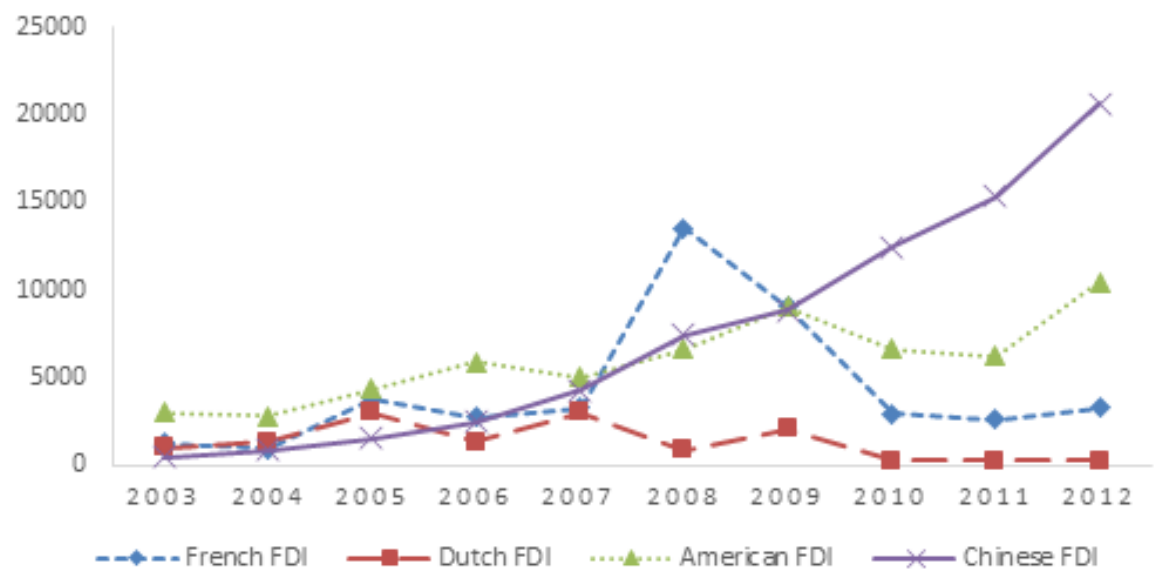

Figure 3.2: FDI Stocks in Africa (in billions Dollars)

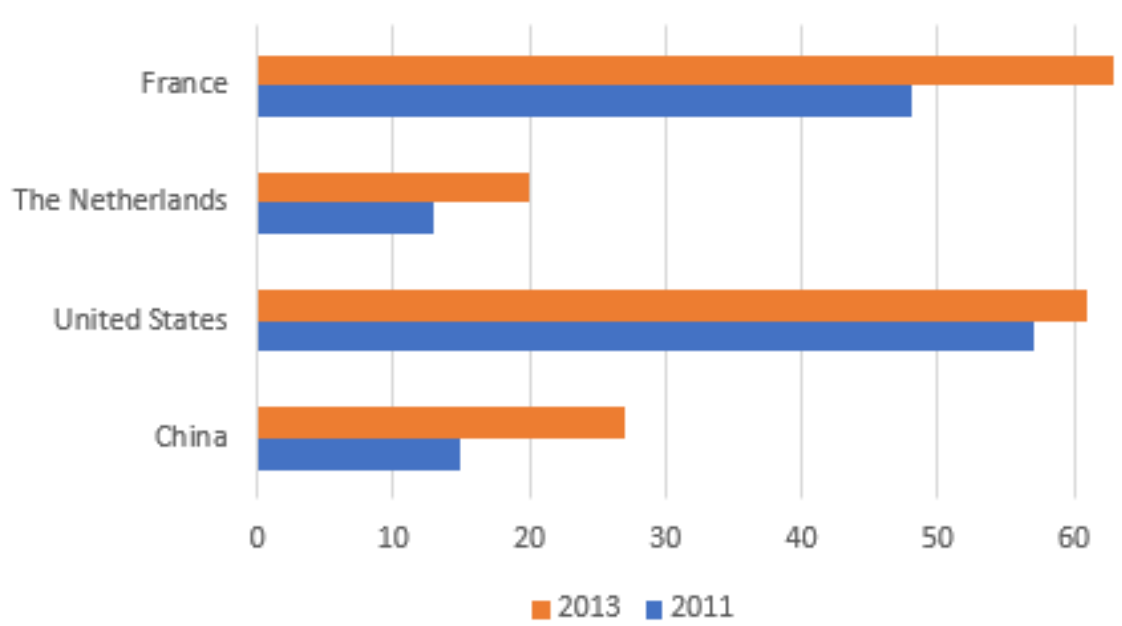

Notes: Constructed by Authors using data from the UNCTAD world investment reports (2014 and 2019) 
Figure 3.3: FDI Percentile Distribution in Africa

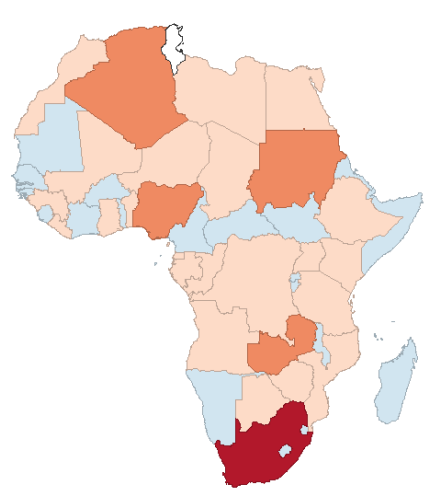

(a) Chinese FDI

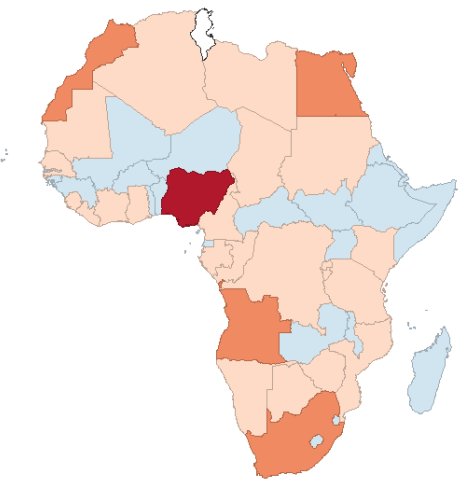

(c) Dutch FDI

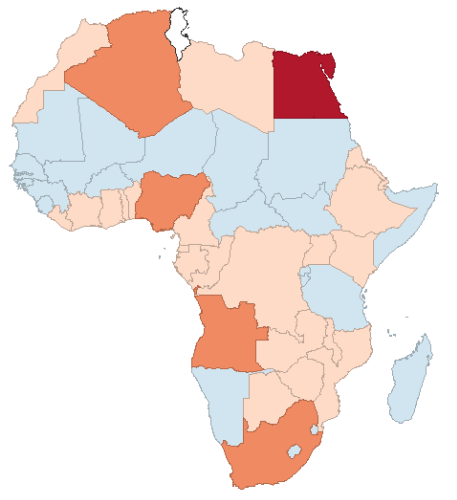

(b) US FDI
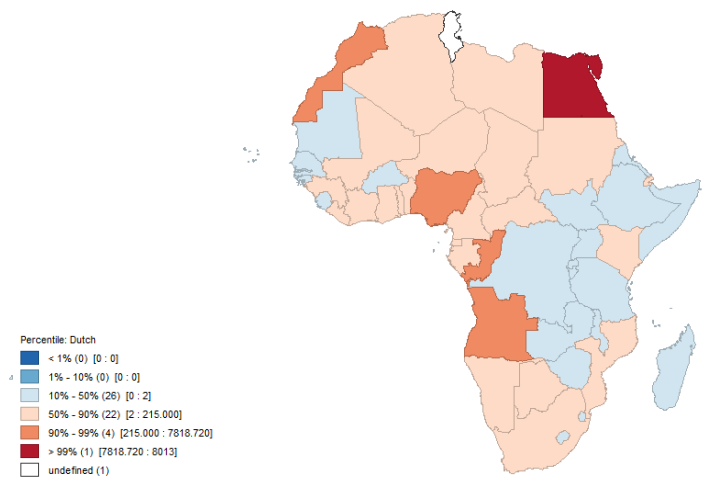

(d) French FDI

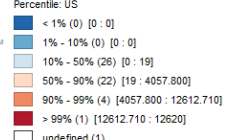

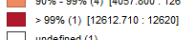

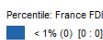

$1 \%-10 \%(1) ; 0: 0$

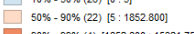

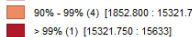

Notes: Constructed by Authors using aggregated data from 2002-2013 
Table 3.1: Foreign Direct Investments Motives

\begin{tabular}{|c|c|c|}
\hline $\begin{array}{l}\text { FDI Mo- } \\
\text { tives }\end{array}$ & Definition & $\begin{array}{l}\text { Sign of the SMP or the } \\
\text { Spatial Lag }\end{array}$ \\
\hline $\begin{array}{l}\text { Horizontal } \\
\text { FDI }\end{array}$ & $\begin{array}{l}\text { Purely horizontal motive illustrate a case where } \\
\text { MNEs try to cut trade and transportation costs } \\
\text { by moving production plants and distribution } \\
\text { centers overseas. }\end{array}$ & $\begin{array}{l}\text { Spatial Lag of FDI (0) and } \\
\text { SMPs }(0)\end{array}$ \\
\hline $\begin{array}{l}\text { Export } \\
\text { Platform } \\
\text { FDI }\end{array}$ & $\begin{array}{l}\text { Export platform FDI arise when the par- } \\
\text { ent country uses the host country to serve } \\
\text { third country markets. This motive occurs when } \\
\text { trade protection between destination markets } \\
\text { is less than frictions between parent and desti- } \\
\text { nation countries. Thus, the effect on a host } \\
\text { country's FDI of an increase in surrounding } \\
\text { countries FDI is negative. Moreover, since all } \\
\text { African countries are a potential destination in } \\
\text { this case, the SMP variables are expected to } \\
\text { have a positive relationship with the host coun- } \\
\text { try FDI. }\end{array}$ & $\begin{array}{l}\text { Spatial Lag of FDI }(-) \text { and } \\
\operatorname{SMP}(+)\end{array}$ \\
\hline $\begin{array}{l}\text { Purely } \\
\text { vertical } \\
\text { FDI }\end{array}$ & $\begin{array}{l}\text { Purely vertical FDI are when parent coun- } \\
\text { try's MNEs are seeking comparative advan- } \\
\text { tages associated with factor cost differences } \\
\text { among African countries.Poelhekke and Van } \\
\text { Der Ploeg (2013) suggest that one need to dif- } \\
\text { ferentiate between non-resource vertical FDI } \\
\text { and resource vertical FDI. }\end{array}$ & $\begin{array}{l}\text { Spatial lag of FDI (-) and } \\
\text { SMP }(0) \text {, but in some cases of } \\
\text { resource FDI, we could have } \\
\text { the Spatial lag of FDI (-) and } \\
\text { SMP (-). }\end{array}$ \\
\hline $\begin{array}{l}\text { Complex } \\
\text { Vertical } \\
\text { motive }\end{array}$ & $\begin{array}{l}\text { FDIs seek to fragment their production in mul- } \\
\text { tiple African countries for factor costs pur- } \\
\text { poses. In this case, FDI of the host country } \\
\text { will have a positive relationship with FDIs from } \\
\text { neighboring countries, and the SMP variables } \\
\text { effects will be positive.However, with interme- } \\
\text { diate levels of border costs between the host } \\
\text { country and its neighbors and a large periph- } \\
\text { eral (not centrally located within the group of } \\
\text { neighboring countries) host market, surround- } \\
\text { ing market potential may have a negative effect } \\
\text { (Poelhekke and Van Der Ploeg, 2013). }\end{array}$ & $\begin{array}{l}\text { Spatial Lag of FDI }(+) \text { and } \\
\operatorname{SMP}(+/ \text { or }-)\end{array}$ \\
\hline
\end{tabular}

Notes: The definitions are collected from previous literature (Regelink and Elhorst, 2015; Poelhekke and Van Der Ploeg, 2013). 
Table 3.2: Data and Sources

\begin{tabular}{|c|c|c|}
\hline Variables & Definition & Data sources \\
\hline $\begin{array}{l}\text { Foreign Direct } \\
\text { Investment (FDI) } \\
\text { France, US, the } \\
\text { Netherland, and } \\
\text { China. }\end{array}$ & $\begin{array}{l}\text { Foreign Direct Investment (FDI) flows record } \\
\text { the value of cross-border transactions related } \\
\text { to direct investment during a given period of } \\
\text { time. }\end{array}$ & $\begin{array}{l}\text { Data for France, US, Nether- } \\
\text { land come from the Orga- } \\
\text { nization for Economic Co- } \\
\text { operation and Development } \\
\text { (OECD). } \\
\text { Data for China come from the } \\
\text { China-Africa Research Initia- } \\
\text { tive from the John Hopkins } \\
\text { University }\end{array}$ \\
\hline GDP growth & $\begin{array}{l}\text { Gross Domestic Product per capita annual } \\
\text { growth rate }\end{array}$ & $\begin{array}{l}\text { World Bank-World Develop- } \\
\text { ment Indicator }\end{array}$ \\
\hline Inflation & $\begin{array}{l}\text { Inflation as measured by the consumer price } \\
\text { index (annual percentage) }\end{array}$ & $\begin{array}{l}\text { International Monetary Fund } \\
\text { (IMF), International Finan- } \\
\text { cial Statistics and data files. }\end{array}$ \\
\hline Trade Openness & Inverse of (exports + imports)/GDP & $\begin{array}{l}\text { World Bank-World Develop- } \\
\text { ment Indicator }\end{array}$ \\
\hline Infrastructure & $\begin{array}{l}\text { Electric power consumption }(\mathrm{kWh} \text { per } \\
\text { capita)-electric power consumption is used } \\
\text { as the proxy for Infrastructure. }\end{array}$ & $\begin{array}{l}\text { World Bank-World Develop- } \\
\text { ment Indicator }\end{array}$ \\
\hline $\begin{array}{l}\text { Natural Resources } \\
\text { Rents }\end{array}$ & $\begin{array}{l}\text { Total resource rents are the sum of oil rents, } \\
\text { natural gas rents, coal rents (hard and soft), } \\
\text { mineral rents, and forest rents, as a percentage } \\
\text { of GDP }\end{array}$ & $\begin{array}{l}\text { World Bank-World Develop- } \\
\text { ment Indicator }\end{array}$ \\
\hline Political Climate & $\begin{array}{l}\text { Government Effectiveness: estimates of percep- } \\
\text { tions of the quality of public services, the qual- } \\
\text { ity of the civil service and the degree of its inde- } \\
\text { pendence from political pressures, the quality } \\
\text { of policy formulation and implementation, and } \\
\text { the credibility of the government's commitment } \\
\text { to such policies. Range }=-2.5 \text { to } 2.5\end{array}$ & $\begin{array}{l}\text { World Bank 's Worldwide } \\
\text { Governance Indicators. This } \\
\text { variable is used as the politi- } \\
\text { cal climate proxy }\end{array}$ \\
\hline Population & Total Population & $\begin{array}{l}\text { World Bank-World Develop- } \\
\text { ment Indicator }\end{array}$ \\
\hline Distance & $\begin{array}{l}\text { Geographic distance between two African cap- } \\
\text { ital cities }\end{array}$ & $\begin{array}{l}\text { Centre d' Etudes Prospectives } \\
\text { et d ' Informations Interna- } \\
\text { tionales (CEPII) }\end{array}$ \\
\hline
\end{tabular}

Notes: Lists of the countries are Algeria, Angola, Benin, Botswana, Burundi, Cameroon, Central African Republic, Chad, Congo, Democratic Republic of Congo, Cote d' Ivoire, Djibouti, Egypt, Equatorial Guinea, Eritrea, Ethiopia, Gabon, Ghana, Guinea, Kenya, Lesotho, Liberia, Libya, Malawi, Mali, Mauritania, Morocco, Mozambique, Namibia, Niger, Nigeria, Rwanda, Senegal, Sierra Leone, South-Africa, Sudan, Tanzania, Togo, Tunisia, Uganda, Zambia, and Zimbabwe. 
Table 3.3: African Countries and Colonial Historical Ties

\begin{tabular}{l|l}
\hline $\begin{array}{l}\text { European } \\
\text { Countries }\end{array}$ & African Countries \\
\hline England & $\begin{array}{l}\text { Egypt, Ghana, Kenya, Malawi, Namibia, } \\
\text { South-Africa, Tanzania, Nigeria, Sierra-Leone, } \\
\text { Uganda, Zambia, Zimbabwe, Botswana, } \\
\text { Lesotho, Liberia }\end{array}$ \\
\hline France & $\begin{array}{l}\text { Algeria, Benin, Cameroon, Central African Re- } \\
\text { public, Chad, Congo, Cote d' Ivoire, Gabon, } \\
\text { Guinea, Niger, Senegal, Togo, Tunisia, Mali, } \\
\text { Djibouti, Morocco }\end{array}$ \\
\hline Belgium & $\begin{array}{l}\text { Burundi, Democratic Republic of Congo, } \\
\text { Rwanda }\end{array}$ \\
\hline Portugal & Angola, Equatorial Guinea, Mozambique \\
\hline Italy & Eritrea, Ethiopia \\
\hline
\end{tabular}

Table 3.4: Summary Statistics

\begin{tabular}{l|l|l}
\hline Variables & Mean & Unit of Measurements \\
\hline French FDI & 102.94 & in million of \$ \\
\hline Dutch FDI & 31.54 & in million of $\$$ \\
\hline American FDI & 142.83 & in million of $\$$ \\
\hline Chinese FDI & 176.61 & in million of $\$$ \\
\hline GDP growth & 5.24 & annual percentage \\
\hline Natural Resources Rent & 15.62 & percentage of the GDP \\
\hline Total Population & $2.18 \mathrm{e}+07$ & in units \\
\hline Trade Openness & 1.16 & in units \\
\hline Inflation & 8.202 & annual percentage \\
\hline Infrastructure & 527.02 & in kilowatts \\
\hline Political Climate & -0.77 & index that ranges between -2.5 to 2.5 \\
\hline
\end{tabular}

Notes: In this table, we only report the summary statistics of the variables used in this study. 
Table 3.5: Determinants of FDI, Fixed Effects Panel Regression

\begin{tabular}{|c|c|c|c|c|}
\hline & \multicolumn{4}{|c|}{$\operatorname{Ln}(F D I)_{i, t}$} \\
\hline & French & Dutch & American & Chinese \\
\hline $\operatorname{Ln}(F D I)_{i, t-1}$ & $\begin{array}{l}0.5721^{\text {*** }} \\
(0.000)\end{array}$ & $\begin{array}{l}0.2762^{* * *} \\
(0.000)\end{array}$ & $\begin{array}{l}0.3811^{* * *} \\
(0.000)\end{array}$ & $\begin{array}{l}0.5287^{* * * *} \\
(0.000)\end{array}$ \\
\hline $\ln (N R E)_{i, t-1}$ & $\begin{array}{l}0.0905^{* *} \\
(0.015)\end{array}$ & $\begin{array}{l}-0.1423^{* * *} \\
(0.007)\end{array}$ & $\begin{array}{l}0.0362 \\
(0.493)\end{array}$ & $\begin{array}{l}0.0348^{* *} \\
(0.036)\end{array}$ \\
\hline $\ln (\text { Open })_{i, t-1}$ & $\begin{array}{l}0.4353 \\
(0.570)\end{array}$ & $\begin{array}{l}1.0338 \\
(0.344)\end{array}$ & $\begin{array}{l}1.7344 \\
(0.0 .117)\end{array}$ & $\begin{array}{l}0.3582 \\
(0.300)\end{array}$ \\
\hline GDPgrowth $h_{i, t-1}$ & $\begin{array}{c}-0.0249 \\
(0.359)\end{array}$ & $\begin{array}{c}-0.0071 \\
(0.852)\end{array}$ & $\begin{array}{c}-0.0065 \\
(0.867)\end{array}$ & $\begin{array}{l}0.0170 \\
(0.0 .165)\end{array}$ \\
\hline $\ln (p o p)_{i, t}$ & $\begin{array}{l}-18.3092^{* * *} \\
(0.002)\end{array}$ & $\begin{array}{l}-21.6161^{* * *} \\
(0.009)\end{array}$ & $\begin{array}{l}-22.0044^{* *} \\
(0.010)\end{array}$ & $\begin{array}{l}2.7373^{*} \\
(0.293)\end{array}$ \\
\hline Infrastructure $_{i, t}$ & $\begin{array}{c}-0.0001 \\
(0.915)\end{array}$ & $\begin{array}{l}0.0012 \\
(0.606)\end{array}$ & $\begin{array}{l}0.0014 \\
(0.566)\end{array}$ & $\begin{array}{c}-0.0006 \\
(0.379)\end{array}$ \\
\hline Inflation $_{i, t}$ & $\begin{array}{c}-0.0020 \\
(0.875)\end{array}$ & $\begin{array}{c}-0.0114 \\
(0.587)\end{array}$ & $\begin{array}{c}-0.0103 \\
(0.575)\end{array}$ & $\begin{array}{l}5.8900 \\
(0.999)\end{array}$ \\
\hline 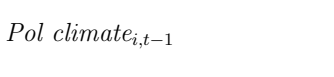 & $\begin{array}{l}1.3309 \\
(0.270)\end{array}$ & $\begin{array}{c}-1.4092 \\
(0.405)\end{array}$ & $\begin{array}{l}3.3168^{* *} \\
(0.050)\end{array}$ & $\begin{array}{l}0.8267 \\
(0.123)\end{array}$ \\
\hline Intercept & $\begin{array}{l}293.1828^{* * *} \\
(0.002)\end{array}$ & $\begin{array}{l}343.869^{* *} \\
(0.010)\end{array}$ & $\begin{array}{l}352.2651^{* * *} \\
(0.009)\end{array}$ & $\begin{array}{l}-44.3770 \\
(0.290)\end{array}$ \\
\hline Year fixed effects & $\checkmark$ & $\checkmark$ & $\checkmark$ & $\checkmark$ \\
\hline African country fixed effects & $\checkmark$ & $\checkmark$ & $\checkmark$ & $\checkmark$ \\
\hline R-Squared & 0.56 & 0.31 & 0.23 & 0.72 \\
\hline Observations & 420 & 420 & 420 & 420 \\
\hline$C D$-test & $\begin{array}{c}13.895^{\text {**** }} \\
(0.000)\end{array}$ & $\begin{array}{c}10.230^{* * *} \\
(0.000)\end{array}$ & $\begin{array}{c}0.923 \\
(0.356)\end{array}$ & $\begin{array}{c}20.920^{* * * *} \\
(0.000)\end{array}$ \\
\hline
\end{tabular}

Statistical significance: "*" 10 percent, "**" 5 percent, "***" 1 percent. $p$-value in parentheses. 
Table 3.6: Spatial Interdependence of FDI Flows, SDM with Historical Colonial Ties Weight Matrix

\begin{tabular}{|c|c|c|c|c|c|c|c|c|c|c|c|c|}
\hline & \multicolumn{12}{|c|}{$\ln (F D I)_{i, t}$} \\
\hline & \multicolumn{3}{|c|}{ French } & \multicolumn{3}{|c|}{ Dutch } & \multicolumn{3}{|c|}{ American } & \multicolumn{3}{|c|}{ Chinese } \\
\hline & $\begin{array}{l}\text { Direct } \\
(1)\end{array}$ & $\begin{array}{c}\text { Indirect } \\
(2)\end{array}$ & $\begin{array}{c}\text { Total } \\
(3)\end{array}$ & $\begin{array}{l}\text { Direct } \\
(4)\end{array}$ & $\begin{array}{c}\text { Indirect } \\
(5)\end{array}$ & $\begin{array}{c}\text { Total } \\
(6)\end{array}$ & $\begin{array}{l}\text { Direct } \\
(7)\end{array}$ & $\begin{array}{c}\text { Indirect } \\
(8)\end{array}$ & $\begin{array}{c}\text { Total } \\
(9)\end{array}$ & $\begin{array}{l}\text { Direct } \\
(10)\end{array}$ & $\begin{array}{c}\text { Indirect } \\
(11)\end{array}$ & $\begin{array}{l}\text { Total } \\
(12)\end{array}$ \\
\hline $\ln (F D I)_{i, t-1}$ & $\begin{array}{c}0.5718^{* * *} \\
(0.000)\end{array}$ & $\begin{array}{l}0.0410 \\
(0.720)\end{array}$ & $\begin{array}{c}0.6129^{* * *} \\
(0.000)\end{array}$ & $\begin{array}{c}0.2456^{* * *} \\
(0.000)\end{array}$ & $\begin{array}{c}0.2326^{* *} \\
(0.055)\end{array}$ & $\begin{array}{l}0.0129 \\
(0.927)\end{array}$ & $\begin{array}{c}0.3684^{* * *} \\
(0.000)\end{array}$ & $\begin{array}{l}0.0285 \\
(0.816)\end{array}$ & $\begin{array}{c}0.3970^{* * * *} \\
(0.001)\end{array}$ & $\begin{array}{c}0.5167^{* * * *} \\
(0.000)\end{array}$ & $\begin{array}{l}-0.1377 \\
(0.243)\end{array}$ & $\begin{array}{c}0.3789^{* *} \\
(0.023)\end{array}$ \\
\hline $\ln (N R E)_{i, t-1}$ & $\begin{array}{c}0.0969^{* * *} \\
(0.007)\end{array}$ & $\begin{array}{c}-0.0392 \\
(0.465)\end{array}$ & $\begin{array}{l}0.0577 \\
(0.358)\end{array}$ & $\begin{array}{c}-0.1597^{* * *} \\
(0.000)\end{array}$ & $\begin{array}{l}0.0194 \\
(0.787)\end{array}$ & $\begin{array}{c}-0.1403^{*} \\
(0.085)\end{array}$ & $\begin{array}{l}0.0173 \\
(0.552)\end{array}$ & $\begin{array}{l}0.2769 \\
(0.000)\end{array}$ & $\begin{array}{c}0.2942^{* * * *} \\
(0.001)\end{array}$ & $\begin{array}{l}0.0364 \\
(0.141)\end{array}$ & $\begin{array}{c}-0.0095 \\
(0.559)\end{array}$ & $\begin{array}{l}0.0268 \\
(0.293)\end{array}$ \\
\hline $\ln (\text { Open })_{i t-1}$ & $\begin{array}{l}0.7199 \\
(0.392)\end{array}$ & $\begin{array}{r}-1.1535 \\
(0.169)\end{array}$ & $\begin{array}{l}-0.4335 \\
(0.490)\end{array}$ & $\begin{array}{l}0.7140 \\
(0.402)\end{array}$ & $\begin{array}{c}3.7689^{* * *} \\
(0.007)\end{array}$ & $\begin{array}{c}4.4829^{* * *} \\
(0.000)\end{array}$ & $\begin{array}{l}0.5451 \\
(0.557)\end{array}$ & $\begin{array}{c}5.0106^{* * *} \\
(0.002)\end{array}$ & $\begin{array}{c}5.5555^{* * * *} \\
(0.000)\end{array}$ & $\begin{array}{l}0.3984^{*} \\
(0.076)\end{array}$ & $\begin{array}{l}-0.4929 \\
(0.291)\end{array}$ & $\begin{array}{l}-0.0944 \\
(0.854)\end{array}$ \\
\hline GDPgrowth $_{i, t-1}$ & $\begin{array}{l}-0.0260 \\
(0.285)\end{array}$ & $\begin{array}{l}-0.0378 \\
(0.380)\end{array}$ & $\begin{array}{l}-0.0639 \\
(0.109)\end{array}$ & $\begin{array}{c}-0.0144 \\
(0.606)\end{array}$ & $\begin{array}{l}0.0223 \\
(0.753)\end{array}$ & $\begin{array}{l}0.0079 \\
(0.915)\end{array}$ & $\begin{array}{l}-0.0246 \\
(0.472)\end{array}$ & $\begin{array}{c}-0.1654^{*} \\
(0.053)\end{array}$ & $\begin{array}{c}-0.1900^{* *} \\
(0.035)\end{array}$ & $\begin{array}{l}0.0176 \\
(0.151)\end{array}$ & $\begin{array}{l}0.0297 \\
(0.246)\end{array}$ & $\begin{array}{c}0.0473^{* *} \\
(0.002)\end{array}$ \\
\hline $\ln (\text { pop })_{i t}$ & $\begin{array}{c}-17.6388 \\
(0.107)\end{array}$ & $\begin{array}{c}-10.1720 \\
(0.472)\end{array}$ & $\begin{array}{c}-27.8109^{*} \\
(0.061)\end{array}$ & $\begin{array}{c}-20.3840 * * * \\
(0.005)\end{array}$ & $\begin{array}{c}-51.2127^{* *} \\
(0.042)\end{array}$ & $\begin{array}{c}-71.5968^{* * *} \\
(0.005)\end{array}$ & $\begin{array}{c}-21.897^{* * *} \\
(0.000)\end{array}$ & $\begin{array}{c}-14.6910 \\
(0.448)\end{array}$ & $\begin{array}{c}-36.5886^{*} \\
(0.060)\end{array}$ & $\begin{array}{l}2.5612 \\
(0.345)\end{array}$ & $\begin{array}{c}11.2827^{* *} \\
(0.048)\end{array}$ & $\begin{array}{c}13.8440^{* *} \\
(0.002)\end{array}$ \\
\hline Infrastructure $_{i t}$ & $\begin{array}{l}0.0002 \\
(0.852)\end{array}$ & $\begin{array}{c}-0.0001 \\
(0.967)\end{array}$ & $\begin{array}{c}0.00006 \\
(0.0 .985)\end{array}$ & $\begin{array}{l}0.0015 \\
(0.306)\end{array}$ & $\begin{array}{c}0.0187^{* *} \\
(0.053)\end{array}$ & $\begin{array}{c}0.0202^{* *} \\
(0.032)\end{array}$ & $\begin{array}{l}0.0015 \\
(0.281)\end{array}$ & $\begin{array}{c}-0.0127 \\
(0.225)\end{array}$ & $\begin{array}{c}-0.0111 \\
(0.264)\end{array}$ & $\begin{array}{c}-0.0007 \\
(0.100)\end{array}$ & $\begin{array}{c}0 .-0.0034 \\
(0.100)\end{array}$ & $\begin{array}{c}0.0041^{* *} \\
(0.050)\end{array}$ \\
\hline Inflation $_{i t}$ & $\begin{array}{c}-0.0031 \\
(0.610)\end{array}$ & $\begin{array}{c}0.017 \\
(0.582)\end{array}$ & $\begin{array}{l}-0.0186 \\
(0.486)\end{array}$ & $\begin{array}{l}0.0098 \\
(0.110)\end{array}$ & $\begin{array}{l}0.0262 \\
(0.504)\end{array}$ & $\begin{array}{l}0.0360 \\
(0.361)\end{array}$ & $\begin{array}{c}-0.0118 \\
(0.102)\end{array}$ & $\begin{array}{l}0.0008 \\
(0.987)\end{array}$ & $\begin{array}{l}0.0110 \\
(0.852)\end{array}$ & $\begin{array}{l}0.0018 \\
(0.604)\end{array}$ & $\begin{array}{c}0.0196^{* *} \\
(0.023)\end{array}$ & $\begin{array}{c}0.0214^{* *} \\
(0.019)\end{array}$ \\
\hline Pol climate $_{i, t}$ & $\begin{array}{l}2.0830^{*} \\
(0.032)\end{array}$ & $\begin{array}{l}4.4243^{*} \\
(0.070)\end{array}$ & $\begin{array}{c}6.5073^{* *} \\
(0.024)\end{array}$ & $\begin{array}{l}-1.0879 \\
(0.548)\end{array}$ & $\begin{array}{l}3.2340 \\
(0.286)\end{array}$ & $\begin{array}{l}2.1461 \\
(0.039)\end{array}$ & $\begin{array}{c}3.4489^{* *} \\
(0.179)\end{array}$ & $\begin{array}{l}5.4436 \\
(0.736)\end{array}$ & $\begin{array}{l}8.8926^{*} \\
(0.073)\end{array}$ & $\begin{array}{c}0.6928^{* *} \\
(0.044)\end{array}$ & $\begin{array}{c}-0.5908 \\
(0.513)\end{array}$ & $\begin{array}{l}0.1020 \\
(0.909)\end{array}$ \\
\hline$W L n(F D I)_{i, t}$ & & $\begin{array}{c}-0.3002^{* * *} * \\
(0.000)\end{array}$ & & & $\begin{array}{c}-0.1462^{* *} \\
(0.015)\end{array}$ & & & $\begin{array}{c}-0.0103 \\
(0.876)\end{array}$ & & & $\begin{array}{c}-0.0950^{*} \\
(0.072)\end{array}$ & \\
\hline Observation & & 420 & & & 420 & & & 420 & & & 420 & \\
\hline R-Squared & & 0.41 & & & 0.20 & & & 0.13 & & & 0.68 & \\
\hline
\end{tabular}

Statistical significance: "*" 10 percent, "**" 5 percent, "***" 1 percent. $p$-value in parentheses. 
Table 3.7: Spatial Crowding In and Crowding Out of FDI Flows, SDM with Historical Colonial Ties Weight Matrix

\begin{tabular}{|c|c|c|c|c|c|c|c|c|c|}
\hline & \multicolumn{9}{|c|}{$\operatorname{Ln}(F D I)_{i, t}$} \\
\hline & \multicolumn{3}{|c|}{ French } & \multicolumn{3}{|c|}{ Dutch } & \multicolumn{3}{|c|}{ American } \\
\hline & $\begin{array}{l}\text { Direct } \\
(1)\end{array}$ & $\begin{array}{c}\text { Indirect } \\
(2)\end{array}$ & $\begin{array}{c}\text { Total } \\
(3)\end{array}$ & $\begin{array}{l}\text { Direct } \\
(4)\end{array}$ & $\begin{array}{c}\text { Indirect } \\
(5)\end{array}$ & $\begin{array}{c}\text { Total } \\
(6)\end{array}$ & $\begin{array}{l}\text { Direct } \\
(7)\end{array}$ & $\begin{array}{c}\text { Indirect } \\
(8)\end{array}$ & $\begin{array}{c}\text { Total } \\
(9)\end{array}$ \\
\hline $\ln (\text { FrenchF } D I)_{i, t-1}$ & $0.5775^{* * *}$ & 0.4770 & $0.6252^{* * *}$ & - & - & - & - & - & - \\
\hline & $(0.000)$ & $(0.700)$ & $(0.000)$ & & & & & & \\
\hline $\ln (\text { DutchFDI })_{i, t-1}$ & - & - & - & $\begin{array}{c}0.2410^{* * * *} \\
(0.000)\end{array}$ & $\begin{array}{c}-0.2145^{*} \\
(0.000)\end{array}$ & $\begin{array}{c}0.0265^{* *} \\
(0.062)\end{array}$ & - & - & - \\
\hline $\ln (\text { AmericanF DI })_{i, t-1}$ & - & - & - & - & - & - & $\begin{array}{c}0.3677^{* * * *} \\
(0.000)\end{array}$ & $\begin{array}{l}0.0394 \\
(0.729)\end{array}$ & $\begin{array}{c}0.4072^{* * *} \\
(0.000)\end{array}$ \\
\hline $\ln (\text { ChineseF DI })_{i, t-1}$ & $\begin{array}{l}-0.0040 \\
(0.968)\end{array}$ & $\begin{array}{l}0.1008 \\
(0.654)\end{array}$ & $\begin{array}{l}0.0967 \\
(0.694)\end{array}$ & $\begin{array}{c}-0.02015^{*} \\
(0.087)\end{array}$ & $\begin{array}{l}-0.1489 \\
(0.457)\end{array}$ & $\begin{array}{l}0.3504 \\
(0.117)\end{array}$ & $\begin{array}{l}0.0741 \\
(0.381)\end{array}$ & $\begin{array}{l}-0.5138 \\
(0.050)\end{array}$ & $\begin{array}{c}-0.5880^{* *} \\
(0.028)\end{array}$ \\
\hline $\ln (N R E)_{i, t-1}$ & $\begin{array}{c}0.1002^{* *} \\
(0.005)\end{array}$ & $\begin{array}{r}-0.0421 \\
(0.446)\end{array}$ & $\begin{array}{l}0.0600 \\
(0.357)\end{array}$ & $\begin{array}{c}-0.1441 * * * \\
(0.001)\end{array}$ & $\begin{array}{l}0.0277 \\
(0.704)\end{array}$ & $\begin{array}{l}-0.1163 \\
(0.146)\end{array}$ & $\begin{array}{l}0.0258 \\
(0.389)\end{array}$ & $\begin{array}{c}0.2970^{* *} \\
(0.000)\end{array}$ & $\begin{array}{c}0.3228^{* * *} \\
(0.000)\end{array}$ \\
\hline $\ln (\text { Open })_{i, t-1}$ & $\begin{array}{l}0.6604 \\
(0.448)\end{array}$ & $\begin{array}{c}-0.9670 \\
(0.381)\end{array}$ & $\begin{array}{c}-0.3065 \\
(0.711)\end{array}$ & $\begin{array}{l}0.8473 \\
(0.329)\end{array}$ & $\begin{array}{c}3.030^{* *} \\
(0.018)\end{array}$ & $\begin{array}{c}3.8780^{* * *} \\
(0.004)\end{array}$ & $\begin{array}{l}0.4357 \\
(0.665)\end{array}$ & $\begin{array}{c}3.8919^{* *} \\
(0.026)\end{array}$ & $\begin{array}{c}4.3277^{* * *} \\
(0.004)\end{array}$ \\
\hline GDPgrowth $_{i, t-1}$ & $\begin{array}{l}-0.0255 \\
(0.276)\end{array}$ & $\begin{array}{l}-0.0397 \\
(0.381)\end{array}$ & $\begin{array}{l}-0.0653 \\
(0.711)\end{array}$ & $\begin{array}{l}-0.0170 \\
(0.504)\end{array}$ & $\begin{array}{l}0.0281 \\
(0.683)\end{array}$ & $\begin{array}{l}0.0111 \\
(0.874)\end{array}$ & $\begin{array}{l}-0.0246 \\
(0.396)\end{array}$ & $\begin{array}{c}-0.1588^{* *} \\
(0.041)\end{array}$ & $\begin{array}{l}-0.0268 \\
(0.023)\end{array}$ \\
\hline $\ln (\text { рор })_{i, t}$ & $\begin{array}{c}-16.1551 \\
(0.173)\end{array}$ & $\begin{array}{c}-12.4113 \\
(0.454)\end{array}$ & $\begin{array}{c}-28.5665^{*} \\
(0.090)\end{array}$ & $\begin{array}{c}-18.5293^{* *} \\
(0.010)\end{array}$ & $\begin{array}{c}-43.5997^{*} \\
(0.076)\end{array}$ & $\begin{array}{c}-62.1290^{* *} \\
(0.012)\end{array}$ & $\begin{array}{c}-20.8282^{* * *} \\
(0.000)\end{array}$ & $\begin{array}{l}-1.2155 \\
(0.448)\end{array}$ & $\begin{array}{c}-22.0437 \\
(0.060)\end{array}$ \\
\hline Infrastructure $_{i t}$ & $\begin{array}{l}0.0002 \\
(0.794)\end{array}$ & $\begin{array}{l}0.0003 \\
(0.923)\end{array}$ & $\begin{array}{l}0.0006 \\
(0.874)\end{array}$ & $\begin{array}{l}0.0013 \\
(0.356)\end{array}$ & $\begin{array}{l}0.0165^{*} \\
(0.079)\end{array}$ & $\begin{array}{l}0.0179^{*} \\
(0.055)\end{array}$ & $\begin{array}{l}0.0016 \\
(0.262)\end{array}$ & $\begin{array}{r}-0.0156 \\
(0.148)\end{array}$ & $\begin{array}{c}-0.0139 \\
(0.176)\end{array}$ \\
\hline Inflation $_{i t}$ & $\begin{array}{l}-0.0035 \\
(0.545)\end{array}$ & $\begin{array}{l}-0.0157 \\
(0.619)\end{array}$ & $\begin{array}{c}-0.0193 \\
(0.531)\end{array}$ & $\begin{array}{l}0.0107 \\
(0.103)\end{array}$ & $\begin{array}{l}0.0422 \\
(0.284)\end{array}$ & $\begin{array}{l}0.0529 \\
(0.173)\end{array}$ & $\begin{array}{c}-0.0102 \\
(0.189)\end{array}$ & $\begin{array}{l}0.0217 \\
(0.711)\end{array}$ & $\begin{array}{l}0.0115 \\
(0.855)\end{array}$ \\
\hline${\text { Pol } \text { climate }_{i, t}}$ & $\begin{array}{c}2.1123^{* *} \\
(0.036)\end{array}$ & $\begin{array}{l}4.2721^{*} \\
(0.079)\end{array}$ & $\begin{array}{c}6.3844^{* *} \\
(0.031)\end{array}$ & $\begin{array}{l}-1.1199 \\
(0.575)\end{array}$ & $\begin{array}{l}2.3007 \\
(0.460)\end{array}$ & $\begin{array}{l}1.1807 \\
(0.070)\end{array}$ & $\begin{array}{l}3.1288^{*} \\
(0.176)\end{array}$ & $\begin{array}{l}5.2026 \\
(0.073)\end{array}$ & $\begin{array}{l}8.3315^{*} \\
(0.044)\end{array}$ \\
\hline$W \operatorname{Ln}(F D I)_{i, t}$ & & $\begin{array}{c}-0.3019^{* * *} \\
(0.000)\end{array}$ & & & $\begin{array}{c}-0.1588^{* * *} \\
(0.004)\end{array}$ & & & $\begin{array}{l}-0.0103 \\
(0.876)\end{array}$ & \\
\hline Observation & & 420 & & & 420 & & & 420 & \\
\hline R-Squared & & 0.41 & & & 0.21 & & & 0.13 & \\
\hline
\end{tabular}


Table 3.8: Spatial Interdependence of FDI Flows, SAR with Historical Colonial Ties Weight Matrix

\begin{tabular}{|c|c|c|c|c|c|c|c|c|c|c|c|c|}
\hline & \multicolumn{12}{|c|}{$\ln (F D I)_{i, t}$} \\
\hline & \multicolumn{3}{|c|}{ French } & \multicolumn{3}{|c|}{ Dutch } & \multicolumn{3}{|c|}{ American } & \multicolumn{3}{|c|}{ Chinese } \\
\hline & $\begin{array}{l}\text { Direct } \\
(1)\end{array}$ & $\begin{array}{c}\text { Indirect } \\
(2)\end{array}$ & $\begin{array}{c}\text { Total } \\
(3) \\
\end{array}$ & $\begin{array}{c}\text { Direct } \\
(4)\end{array}$ & $\begin{array}{c}\text { Indirect } \\
(5)\end{array}$ & $\begin{array}{c}\text { Total } \\
(6)\end{array}$ & $\begin{array}{c}\text { Direct } \\
(7)\end{array}$ & $\begin{array}{c}\text { Indirect } \\
(8)\end{array}$ & $\begin{array}{c}\text { Total } \\
(9)\end{array}$ & $\begin{array}{c}\text { Direct } \\
(10)\end{array}$ & $\begin{array}{c}\text { Indirect } \\
(11)\end{array}$ & $\begin{array}{c}\text { Total } \\
(12)\end{array}$ \\
\hline $\ln (F D I)_{i, t-1}$ & $\begin{array}{c}0.5726^{* * *} \\
(0.000)\end{array}$ & $\begin{array}{c}-0.0734^{* *} \\
(0.108)\end{array}$ & $\begin{array}{c}0.4991^{* * * *} \\
(0.000)\end{array}$ & $\begin{array}{c}0.2784^{* * *} \\
(0.000)\end{array}$ & $\begin{array}{l}-0.0082 \\
(0.689)\end{array}$ & $\begin{array}{c}0.2701^{* * *} \\
(0.000)\end{array}$ & $\begin{array}{c}0.3803^{* * *} \\
(0.000)\end{array}$ & $\begin{array}{l}0.0514 \\
(0.816)\end{array}$ & $\begin{array}{c}0.4317^{* * * *} \\
(0.001)\end{array}$ & $\begin{array}{c}0.5288^{* * *} \\
(0.000)\end{array}$ & $\begin{array}{l}-0.0301 \\
(0.188)\end{array}$ & $\begin{array}{c}0.4986^{* * * *} \\
(0.000)\end{array}$ \\
\hline $\ln (N R E)_{i, t-1}$ & $\begin{array}{c}0.0832 * * \\
(0.023)\end{array}$ & $\begin{array}{l}-0.0105 \\
(0.123)\end{array}$ & $\begin{array}{c}0.0726^{* *} \\
(0.028)\end{array}$ & $\begin{array}{c}-0.1492 * * * \\
(0.002)\end{array}$ & $\begin{array}{l}0.0046 \\
(0.676)\end{array}$ & $\begin{array}{c}-0.1380^{* * *} \\
(0.002)\end{array}$ & $\begin{array}{l}0.0258 \\
(0.460)\end{array}$ & $\begin{array}{l}0.0033 \\
(0.557)\end{array}$ & $\begin{array}{l}0.0292 \\
(0.463)\end{array}$ & $\begin{array}{l}0.0336 \\
(0.180)\end{array}$ & $\begin{array}{l}-0.0019 \\
(0.405)\end{array}$ & $\begin{array}{l}0.0317 \\
(0.182)\end{array}$ \\
\hline $\ln (\text { Open })_{i t-1}$ & $\begin{array}{l}0.5632 \\
(0.392)\end{array}$ & $\begin{array}{l}-0.0783 \\
(0.169)\end{array}$ & $\begin{array}{l}0.4849 \\
(0.490)\end{array}$ & $\begin{array}{l}1.1472 \\
(0.182)\end{array}$ & $\begin{array}{l}-0.101 \\
(0.922)\end{array}$ & $\begin{array}{l}1.1371 \\
(0.196)\end{array}$ & $\begin{array}{l}1.6099^{*} \\
(0.075)\end{array}$ & $\begin{array}{l}0.2081 \\
(0.250)\end{array}$ & $\begin{array}{l}1.8180^{*} \\
(0.076)\end{array}$ & $\begin{array}{l}0.3540^{*} \\
(0.084)\end{array}$ & $\begin{array}{c}-0.0224 \\
(0.337)\end{array}$ & $\begin{array}{c}0.3540^{* *} \\
(0.083)\end{array}$ \\
\hline GDPgrowth $_{i, t-1}$ & $\begin{array}{l}-0.0223 \\
(0.378)\end{array}$ & $\begin{array}{l}0.0030 \\
(0.455)\end{array}$ & $\begin{array}{l}-0.0193 \\
(0.379)\end{array}$ & $\begin{array}{l}0.0060 \\
(0.814)\end{array}$ & $\begin{array}{l}0.0006 \\
(0.975)\end{array}$ & $\begin{array}{r}-0.0059 \\
(0.811)\end{array}$ & $\begin{array}{l}-0.0058 \\
(0.863)\end{array}$ & $\begin{array}{r}-0.0008 \\
(0.872)\end{array}$ & $\begin{array}{l}-0.0067 \\
(0.862)\end{array}$ & $\begin{array}{l}0.0175 \\
(0.147)\end{array}$ & $\begin{array}{r}-0.0009 \\
(0.394)\end{array}$ & $\begin{array}{l}0.0166 \\
(0.151)\end{array}$ \\
\hline $\ln (\text { рор })_{i, t}$ & $\begin{array}{c}-20.6242^{*} \\
(0.062)\end{array}$ & $\begin{array}{l}2.7856 \\
(0.170)\end{array}$ & $\begin{array}{c}-17.8386^{*} \\
(0.063)\end{array}$ & $\begin{array}{c}-21.5343^{* * *} \\
(0.006)\end{array}$ & $\begin{array}{l}0.6648 \\
(0.683)\end{array}$ & $\begin{array}{c}-21.5343^{* * *} \\
(0.006)\end{array}$ & $\begin{array}{c}-21.9661^{* * *} \\
(0.001)\end{array}$ & $\begin{array}{c}-2.9714 \\
(0.135)\end{array}$ & $\begin{array}{c}-24.9375^{* * *} \\
(0.001)\end{array}$ & $\begin{array}{l}2.7086 \\
(0.276)\end{array}$ & $\begin{array}{c}-0.1694 \\
(0.464)\end{array}$ & $\begin{array}{l}2.5392 \\
(0.275)\end{array}$ \\
\hline Infrastructure $_{i t}$ & $\begin{array}{c}-0.00005 \\
(0.965)\end{array}$ & $\begin{array}{c}0.00001 \\
(0.908)\end{array}$ & $\begin{array}{l}0.00003 \\
(0.975)\end{array}$ & $\begin{array}{l}0.0012 \\
(0.380)\end{array}$ & $\begin{array}{c}-0.0003 \\
(0.805)\end{array}$ & $\begin{array}{l}0.0012 \\
(0.982)\end{array}$ & $\begin{array}{l}0.0014 \\
(0.334)\end{array}$ & $\begin{array}{l}0.0002 \\
(0.453)\end{array}$ & $\begin{array}{l}0.0017 \\
(0.337)\end{array}$ & $\begin{array}{c}-0.0006^{*} \\
(0.096)\end{array}$ & $\begin{array}{l}0.0004 \\
(0.353)\end{array}$ & $\begin{array}{c}-0.0006^{*} \\
(0.097)\end{array}$ \\
\hline Inflation $_{i t}$ & $\begin{array}{l}-0.0008 \\
(0.884)\end{array}$ & $\begin{array}{l}0.0002 \\
(0.758)\end{array}$ & $\begin{array}{r}-0.0005 \\
(0.909)\end{array}$ & $\begin{array}{l}0.0118 \\
(0.110)\end{array}$ & $\begin{array}{c}-0.0038 \\
(0.701)\end{array}$ & $\begin{array}{l}0.0114 \\
(0.109)\end{array}$ & $\begin{array}{c}-0.0104 \\
(0.100)\end{array}$ & $\begin{array}{c}-0.0014 \\
(0.0014)\end{array}$ & $\begin{array}{l}0.0118 \\
(0.105)\end{array}$ & $\begin{array}{l}0.0001 \\
(0.958)\end{array}$ & $\begin{array}{c}-0.00003 \\
(0.859)\end{array}$ & $\begin{array}{l}0.0001 \\
(0.966)\end{array}$ \\
\hline Pol climate $_{i, t}$ & $\begin{array}{l}1.6115^{*} \\
(0.088) \\
\end{array}$ & $\begin{array}{r}-0.2090 \\
(0.212)\end{array}$ & $\begin{array}{l}1.4025^{*} \\
(0.089)\end{array}$ & $\begin{array}{r}-1.3338 \\
(0.443) \\
\end{array}$ & $\begin{array}{l}0.0223 \\
(0.894) \\
\end{array}$ & $\begin{array}{r}-1.3115 \\
(0.038) \\
\end{array}$ & $\begin{array}{l}3.780^{* *} \\
(0.038) \\
\end{array}$ & $\begin{array}{l}0.4948 \\
(0.235) \\
\end{array}$ & $\begin{array}{c}3.8728^{* *} \\
(0.047) \\
\end{array}$ & $\begin{array}{c}0.7946^{* *} \\
(0.046)\end{array}$ & $\begin{array}{c}-0.0503 \\
(0.305)\end{array}$ & $\begin{array}{c}0.7442^{* *} \\
(0.043) \\
\end{array}$ \\
\hline$W L n(F D I)_{i, t}$ & & $\begin{array}{c}-0.1559^{* *} \\
(0.040)\end{array}$ & & & $\begin{array}{c}-0.0405^{* *} \\
(0.598)\end{array}$ & & & $\begin{array}{l}0.0112^{*} \\
(0.091)\end{array}$ & & & $\begin{array}{l}-0.0654 \\
(0.177)\end{array}$ & \\
\hline LR test & & $\begin{array}{c}14.3094^{* * *} \\
(0.000)\end{array}$ & & & $\begin{array}{c}16.2195^{* * * *} \\
(0.000)\end{array}$ & & & $\begin{array}{c}15.2479^{* * * *} \\
(0.000)\end{array}$ & & & $\begin{array}{c}6.4289^{* *} \\
(0.040)\end{array}$ & \\
\hline Observation & & 420 & & & 420 & & & 420 & & & 420 & \\
\hline R-Squared & & 0.43 & & & 0.20 & & & 0.15 & & & 0.66 & \\
\hline
\end{tabular}


Table 3.9: Spatial Interdependence of FDI Flows, SDM with Distance Weight Matrix

\begin{tabular}{|c|c|c|c|c|c|c|c|c|c|c|c|c|}
\hline & \multicolumn{12}{|c|}{$\ln (F D I)_{i, t}$} \\
\hline & \multicolumn{3}{|c|}{ French } & \multicolumn{3}{|c|}{ Dutch } & \multicolumn{3}{|c|}{ American } & \multicolumn{3}{|c|}{ Chinese } \\
\hline & $\begin{array}{l}\text { Direct } \\
(1)\end{array}$ & $\begin{array}{c}\text { Indirect } \\
(2)\end{array}$ & $\begin{array}{c}\text { Total } \\
(3) \\
\end{array}$ & $\begin{array}{l}\text { Direct } \\
(4)\end{array}$ & $\begin{array}{c}\text { Indirect } \\
(5)\end{array}$ & $\begin{array}{c}\text { Total } \\
(6)\end{array}$ & $\begin{array}{l}\text { Direct } \\
(7)\end{array}$ & $\begin{array}{c}\text { Indirect } \\
(8)\end{array}$ & $\begin{array}{c}\text { Total } \\
(9)\end{array}$ & $\begin{array}{c}\text { Direct } \\
(10)\end{array}$ & $\begin{array}{c}\text { Indirect } \\
(11)\end{array}$ & $\begin{array}{l}\text { Total } \\
(12)\end{array}$ \\
\hline $\ln (F D I)_{i, t-1}$ & $\begin{array}{c}0.5603^{* * * *} \\
(0.000)\end{array}$ & $\begin{array}{c}-0.2075^{* * *} \\
(0.000)\end{array}$ & $\begin{array}{c}0.3528^{* * * *} \\
(0.000)\end{array}$ & $\begin{array}{c}0.2843^{* * * *} \\
(0.000)\end{array}$ & $\begin{array}{l}-0.0099 \\
(0.925)\end{array}$ & $\begin{array}{c}0.2744^{* *} \\
(0.014)\end{array}$ & $\begin{array}{c}0.3751^{* * *} \\
(0.000)\end{array}$ & $\begin{array}{l}-0.0033 \\
(0.974)\end{array}$ & $\begin{array}{c}0.3717^{* * * *} \\
(0.003)\end{array}$ & $\begin{array}{c}0.5288^{* * * *} \\
(0.000)\end{array}$ & $\begin{array}{l}-0.0301 \\
(0.188)\end{array}$ & $\begin{array}{c}0.4986^{* * * *} \\
(0.000)\end{array}$ \\
\hline $\ln (N R E)_{i, t-1}$ & $\begin{array}{c}0.0840^{* *} \\
(0.023)\end{array}$ & $\begin{array}{l}0.0199 \\
(0.771)\end{array}$ & $\begin{array}{l}0.1040 \\
(0.169)\end{array}$ & $\begin{array}{c}-0.1425 * * * \\
(0.005)\end{array}$ & $\begin{array}{l}-0.1629 \\
(0.104)\end{array}$ & $\begin{array}{c}-0.3055^{* *} \\
(0.013)\end{array}$ & $\begin{array}{l}0.0905 \\
(0.655)\end{array}$ & $\begin{array}{l}0.0736 \\
(0.435)\end{array}$ & $\begin{array}{l}0.0169 \\
(0.361)\end{array}$ & $\begin{array}{l}0.0336 \\
(0.180)\end{array}$ & $\begin{array}{l}-0.0454 \\
(0.138)\end{array}$ & $\begin{array}{c}0.0317^{* *} \\
(0.041)\end{array}$ \\
\hline $\ln (\text { Open })_{i t-1}$ & $\begin{array}{l}0.2735 \\
(0.730)\end{array}$ & $\begin{array}{l}-0.9715 \\
(0.397)\end{array}$ & $\begin{array}{c}-0.6979 \\
(0.617)\end{array}$ & $\begin{array}{l}0.9787 \\
(0.320)\end{array}$ & $\begin{array}{l}0.5712 \\
(0.663)\end{array}$ & $\begin{array}{l}1.5499 \\
(0.196)\end{array}$ & $\begin{array}{l}1.1346 \\
(0.201)\end{array}$ & $\begin{array}{c}3.0222^{* *} \\
(0.035)\end{array}$ & $\begin{array}{l}4.1568^{* *} \\
(0.010)\end{array}$ & $\begin{array}{l}0.3540^{*} \\
(0.084)\end{array}$ & $\begin{array}{l}-0.0224 \\
(0.337)\end{array}$ & $\begin{array}{c}0.3540^{* *} \\
(0.083)\end{array}$ \\
\hline GDPgrowth $_{i, t-1}$ & $\begin{array}{r}-0.0280 \\
(0.265)\end{array}$ & $\begin{array}{r}-0.0228 \\
(0.608)\end{array}$ & $\begin{array}{l}-0.0508 \\
(0.271)\end{array}$ & $\begin{array}{r}-0.0049 \\
(0.835)\end{array}$ & $\begin{array}{l}0.0208 \\
(0.709)\end{array}$ & $\begin{array}{l}-0.0258 \\
(0.665)\end{array}$ & $\begin{array}{l}-0.0083 \\
(0.791)\end{array}$ & $\begin{array}{l}0.0077 \\
(0.885)\end{array}$ & $\begin{array}{l}-0.0005 \\
(0.993)\end{array}$ & $\begin{array}{l}0.0175 \\
(0.147)\end{array}$ & $\begin{array}{c}-0.0009 \\
(0.394)\end{array}$ & $\begin{array}{l}0.0166 \\
(0.151)\end{array}$ \\
\hline $\ln (p o p)_{i, t}$ & $\begin{array}{c}-17.3175 \\
(0.114)\end{array}$ & $\begin{array}{l}-0.6765 \\
(0.963)\end{array}$ & $\begin{array}{c}-17.9941^{* *} \\
(0.086)\end{array}$ & $\begin{array}{c}-10.9061 \\
(0.244)\end{array}$ & $\begin{array}{c}-24.4736 \\
(0.709)\end{array}$ & $\begin{array}{c}-35.3797^{* *} \\
(0.065)\end{array}$ & $\begin{array}{c}-16.0204^{* *} \\
(0.021)\end{array}$ & $\begin{array}{c}-12.9647 \\
(0.194)\end{array}$ & $\begin{array}{c}-28.9852^{* * * *} \\
(0.004)\end{array}$ & $\begin{array}{l}2.7086 \\
(0.276)\end{array}$ & $\begin{array}{l}-0.1694 \\
(0.464)\end{array}$ & $\begin{array}{l}2.5392 \\
(0.275)\end{array}$ \\
\hline Infrastructure $_{i t}$ & $\begin{array}{c}-0.00005 \\
(0.965)\end{array}$ & $\begin{array}{l}0.00001 \\
(0.908)\end{array}$ & $\begin{array}{l}0.00003 \\
(0.975)\end{array}$ & $\begin{array}{l}0.0017 \\
(0.356)\end{array}$ & $\begin{array}{l}0.0055 \\
(0.112)\end{array}$ & $\begin{array}{l}0.0072 \\
(0.123)\end{array}$ & $\begin{array}{l}0.0011 \\
(0.531)\end{array}$ & $\begin{array}{l}-0.0005 \\
(0.797)\end{array}$ & $\begin{array}{l}0.0005 \\
(0.840)\end{array}$ & $\begin{array}{c}-0.0006^{*} \\
(0.096)\end{array}$ & $\begin{array}{l}0.0004 \\
(0.353)\end{array}$ & $\begin{array}{c}-0.0006^{*} \\
(0.097)\end{array}$ \\
\hline Inflation $_{i t}$ & $\begin{array}{c}-0.0008 \\
(0.536)\end{array}$ & $\begin{array}{l}0.0018 \\
(0.296)\end{array}$ & $\begin{array}{l}0.0026 \\
(0.171)\end{array}$ & $\begin{array}{c}0.106 \\
(0.000)\end{array}$ & $\begin{array}{c}0.0667^{* * * *} \\
(0.000)\end{array}$ & $\begin{array}{l}0.0106 \\
(0.221)\end{array}$ & $\begin{array}{c}-0.0114^{*} \\
(0.207)\end{array}$ & $\begin{array}{c}-0.0383 \\
(0.306)\end{array}$ & $\begin{array}{l}-0.0497 \\
(0.094)\end{array}$ & $\begin{array}{l}0.0001 \\
(0.958)\end{array}$ & $\begin{array}{c}-0.00003 \\
(0.859)\end{array}$ & $\begin{array}{l}0.0001 \\
(0.966)\end{array}$ \\
\hline Pol climate $_{i, t}$ & $\begin{array}{l}0.4579 \\
(0.632)\end{array}$ & $\begin{array}{c}-6.5598^{* * *} \\
(0.005)\end{array}$ & $\begin{array}{c}-6.1018^{* *} \\
(0.015)\end{array}$ & $\begin{array}{l}-1.3490 \\
(0.459)\end{array}$ & $\begin{array}{l}-1.7017 \\
(0.645)\end{array}$ & $\begin{array}{l}-3.5007 \\
(0.485)\end{array}$ & $\begin{array}{c}3.2400^{*} \\
(0.086)\end{array}$ & $\begin{array}{c}-4.1794 \\
(0.315)\end{array}$ & $\begin{array}{l}-0.9393 \\
(0.849)\end{array}$ & $\begin{array}{c}0.7946^{* *} \\
(0.046)\end{array}$ & $\begin{array}{l}-0.0503 \\
(0.305)\end{array}$ & $\begin{array}{c}0.7442^{* *} \\
(0.043)\end{array}$ \\
\hline$W \operatorname{Ln}(F D I)_{i, t}$ & & $\begin{array}{c}-0.0526^{*} \\
(0.060)\end{array}$ & & & $\begin{array}{r}-0.0658 \\
(0.320)\end{array}$ & & & $\begin{array}{l}0.0748 \\
(0.117)\end{array}$ & & & $\begin{array}{c}-0.0480^{*} \\
(0.092)\end{array}$ & \\
\hline Observation & & 420 & & & 420 & & & 420 & & & 420 & \\
\hline R-Squared & & 0.46 & & & 0.24 & & & 0.15 & & & 0.65 & \\
\hline
\end{tabular}

Statistical significance: "*" 10 percent, "**" 5 percent, "***" 1 percent. $p$-value in parentheses. 


\section{Chapter 4}

\section{The Localized Impacts of Local}

\section{Ethanol Production on Land Use}

\section{Changes: Evidence from Illinois}

\section{and Indiana}

\subsection{Introduction}

Towards the end of the 20th century into the 21th century we saw a number of amendments made to the Energy Policy Act of 1992. The purpose of this federal law is to reduce United States (U.S.) dependence on petroleum fuel and encouraged the U.S. Department of Energy (DOE) to incorporate alternative fuels in its regulatory and voluntary activities. Amendments included the Energy Conservation and Reauthorization Act of 1998 and the Energy Policy Act of 2005. These acts contained provisions for renewable fuel uses and infrastructure development. Another important aspect of these amendments was to reduce the levels of greenhouse gases (GHGs) relative to the petroleum fuel it replaced (Searchinger et al., 2008). 
The 2007 Energy Independence and Security Act was the final amendment that included provisions to increase the supply of renewable fuel sources utilized in the transportation sector. Renewable Fuel Standards (RFS) mandated an annual production of 15 billion gallon of ethanol by 2015, and 36 billion by 2022 (Bracmort, 2015). The legislation pushed cellulosic ethanol to be a large part of the 36 billion mandate, but technology has not been shown to be economically or technically viable to date (Chen et al., 2021). With these biofuel friendly policies in place, the production of ethanol in the U.S. started booming, particularly since 2007 (Figure 4.1). The ethanol production shown in Figure 4.1 is almost all derived from corn.

The RFS has had many impacts on the agricultural and environmental sectors in the U.S. RFS policies have been linked to: conversion of natural land into cropland (Ifft et al., 2019; Lark et al., 2015), changes of commodity prices (Roberts and Schlenker, 2013; Carter et al., 2017) , and shifts in crop rotations sequences (Secchi et al., 2011). Many of these studies have investigated land use at the extensive margin (conversion of non-cropland into cropland) and have mainly relied on simulations and Geographic Information Systems (GIS) methods (Chen and Khanna, 2018; Searchinger et al., 2008; Wright et al., 2017). A few exceptions are studies that have relied on causal effects methods (Ifft et al., 2019; Motamed et al., 2016; Arora et al., 2016). Questions have been raised about studies using causal effects methods based upon issues such as the violation of the parallel trends assumption (for Difference in Difference (DID) methods) and the validity of the instruments (for instrumental variable techniques)(Ifft et al., 2019).

To better understand land use changes at the extensive margin, this research investigates changes in land enrolled in the Conservation Reserve Program $(\mathrm{CRP})^{1}$ (the world's largest conservation program), and total planted corn ${ }^{2}$ acreage in Illinois

\footnotetext{
${ }^{1}$ The Conservation Reserve Program offers a number of environmental benefits. In exchange for a yearly rental payment, farmers agree to retire environmentally sensitive land from agricultural production and plant species that will improve environmental health and quality. Converting this land into cropland for ethanol production purposes would cause a number of these environmental benefits to be lost (Hendricks and Er, 2018).

${ }^{2}$ While Li et al. (2019) find that corn acreage and total acreage are fairly inelastic with respect to changes in ethanol capacity in the vicinity, Motamed et al. (2016) find that corn acreage grew
} 
and Indiana, two of the largest ethanol producing states in America (see Figure 4.2), after the ethanol mandate of 2007. Two states, Illinois and Indiana,will be examined to evaluate the effects that increased ethanol production has had on land uses in one state where production started in early 1970s (Illinois) ${ }^{3}$, and in another state where production did not start until 2007 (Indiana) ${ }^{4}$.

While land use policy is controlled by state and local governments (Wu and Cho, 2007), studies investigating the effects of ethanol production on land use changes have mainly focused on larger geographical scales such as regions and countries (Chen and Khanna, 2018; Ifft et al., 2019). These global effects are important for the federal government that has interests in the trends of land use conversions, nonetheless, land use conversions are still a local political matter (Wu and Cho, 2007). Arora et al. (2016) try to look at this problem at a smaller geographical scale (county level) and try to understand the local effects of the advent of an ethanol plant on cropland conversion in the Dakotas, however, this study suffer from the violation of the parallel trend assumption highlighted in the previous paragraph.

Using the RFS mandate of 2007 as a natural experiment, we find that there are spatial and temporal heterogeneous effects of local ethanol plants on enrolled CRP and total planted corn acres by state. Unlike the single-point estimates derived from DID models at the state level that show that increases in both enrolled CRP acres and total planted corn acres after 2007, SCM estimates show variation at the county and different post-intervention periods. Therefore, disaggregated post-effects at the county level show heterogeneous effects, which would otherwise be missed when relying on single-point estimates that are often used in past studies (Chen and Khanna, 2018; Li et al., 2019; Motamed et al., 2016).

In addition, we find that for both Illinois and Indiana, post-effects for enrolled CRP acres had consistent anticipatory effects. To solve this issue, we backdated our

as a result of biofuel production. If more land got converted into corn production as a result of the RFS mandate, then there would be an increase in greenhouse gas emissions due to this activity.

${ }^{3}$ https://ima-net.org/ethanol-industry-impact-tops-5-5-billion-in-illinois/

${ }^{4}$ https://www.in.gov/oed/agrienergy/ethanol/ 
intervention year to 2002, and in some cases to 2005 as suggested in Abadie (2021) to avoid these anticipatory effects. Overall, we find that the results vary by county and year. As a consequence, these results show the importance of studying land use at the extensive margin on a case-by-case basis since we find evidence of land use at the extensive margin in some post-effects years, but not in others. In the end, we caution against relying on global effects such as those found in (Li et al., 2019; Motamed et al., 2016; Chen and Khanna, 2018), if one wants to understand the local effects of ethanol production on land uses changes. Instead, the synthetic control method offers a case-by-case analysis, which we believe is more suited to local land-use policies.

This study contributes to the literature in two ways. First, we used Synthetic Control Method to capture the local effects of ethanol plants on enrolled CRP and total planted corn acres. To our knowledge, this has not been done in the literature. Second, we discuss the shortcomings of relying on single-point estimates as compared to treating each ethanol-producing county as a case study. The next Section 4.2 discusses related literature that has tried to analyze land-use changes as a result of ethanol production-findings and critiques are presented. In Section 4.3, we discuss our empirical methods. Section 4.4 presents the results of this study, and finally, Section 4.5 and 4.6 discuss these results and concludes this research.

\subsection{Related Literature}

A number of previous studies have examined the impact of ethanol plant locations on total planted corn. For example, Li et al. (2019) study the effects of ethanol plant proximity and crop prices on corn acreage in the contiguous United States. They find that an increase in ethanol capacity led to a 3 percent increase in corn acreage, leading the authors to conclude that corn acreage was inelastic with respect to changes in ethanol capacity in the vicinity. Examining the response that corn production had to local booms of ethanol markets, Motamed et al. (2016) used the US railroad network as an instrumental variable and find that total corn and overall acreage, not only 
grew in already-cultivated areas but also expanded into previously uncultivated areas. Thus, both studies have documented that there was a national issue of land use at extensive margin created by the increase in ethanol production.

Numerous other studies have studied the impact that the production of ethanol has had on land enrolled in the Conservation Reserve Program (CRP) and non-cropland. Chen and Khanna (2018) find that between 2007 and 2012, there was a conversion of 3.2 million acres of unused cropland, with one million of that land coming from the CRP program. Further studying cropland conversion due to ethanol production increase, Wright et al. (2017) find that between 2008 and 2012, about 4.2 million acres of arable non-cropland converted to cropland in the United States. Using a DID method, Ifft et al. (2019) investigate the effect of ethanol plants capacity on CRP land before and after the 2005 and 2007 ethanol mandates and find that less land left the CRP program in ethanol intensive areas. They explain that this is due to the changes that occurred in the CRP design around the same time as the ethanol mandates. It is worth noting that these results are the opposite of Chen and Khanna (2018); Wright et al. (2017) findings.

Despite the fact that land use policy is led by local governments (Wu and Cho, 2007), most of the studies that have analyzed land use changes created by local ethanol production gave us a global, national, or regional picture. Searchinger et al. (2008) use a worldwide agricultural model and find that an ethanol production increase of 56 billion liters, diverted 12.8 million ha of U.S. cropland, and brought 2.8 million ha of additional land into cultivation in Brazil, 2.3 million ha in China, and 2.2 million ha in the United States. Chen and Khanna (2018) use a dynamic, partial equilibrium economic model for the whole United States to understand how the increase in corn ethanol production affected the CRP program. Via high resolution satellite images, Wright and Wimberly (2013) show a decline in total grassland area in the corn belt region during the period of 2006 and 2011, though this decline is explained to be caused by crop prices rather than the ethanol mandates.

Similarly, Ifft et al. (2019) use a county level DID model to study how the conser- 
vation reserve program (CRP) reacted to the RFS mandates in the Midwest. Through a state level study, Brown et al. (2014) investigate land use/land-cover (LULC) data to understand corn demand shifter related to ethanol production in Kansas. In this literature, global estimates are given but it is difficult to pinpoint which areas are affected and whether the sign of the global estimates are the same across other smaller geographical scales.

Overall, only Arora et al. (2016) try to capture the localized effects of ethanol plants. In this study, they use the advent of an ethanol plant in the Dakotas as a natural experiment and use the difference in difference (DID) method to understand the pre and post land use patterns. They find that effects vary by ethanol plants, making the case against the single point estimates for all ethanol plants in a region that are usually provided in the literature. However, in Arora et al. (2016), the parallel trend assumption of the DID model does not hold, and they conclude that identifying the localized effects of the advent of an ethanol plant on land use changes is a challenging task.

The violation of the parallel trend in studies that have used the DID model is a recurring issue. Ifft et al. (2019) also notes that although not violated in their CRP models, the parallel trend assumption does not hold for corn production models.In most land use changes and ethanol production papers, two issues arise when one is trying to implement the DID, the first one being the concurrent crop prices increase around the global crises of 2008, and the ethanol plants construction after 2007. The second one is that ethanol plant tend to locate in areas where corn or other biofuel crops have higher returns - stressing the non-randomness of the location of these plants (Ifft et al., 2019; Towe and Tra, 2013).

In fact, Ifft et al. (2019) also question the validity of the instrument used in Motamed et al. (2016) by explaining that since corn production was growing more rapidly in areas where ethanol plants were built or expanded, there may be a spurious correlation between the ethanol mandate and production decisions-highlighting that other causal inference papers that used methods that are different from the DID 
method may have suffered similar econometrics issues.

Collectively, previous research have failed to offer the localized effects of ethanol production on land use changes. Even studies that have tackled this issue failed to provide plausible estimates due to econometrics issues.

\subsection{Empirical Strategies}

\subsubsection{Case Studies, Data and States Estimates}

We begin this section by discussing our data and the set-up of the quasi-experimental study. Our states of interest in this study are Illinois and Indiana from 2000-2017. Per the US DOE, 12 out 13 top ethanol producing states are located in the Midwest. Figure 4.2 shows that Illinois and Indiana in 2020 were among the top 6 ethanol fuel producing states in the country. If we consider that most ethanol producing counties in the United States have similar characteristics (such as being in the Midwest), studying the local effects of ethanol producing counties in Illinois and Indiana on land use changes can give us some signal of land use at the extensive margin created by local ethanol production activities.

Figures 4.3 and 4.4 represent the counties that have an ethanol plant according to the Renewable Fuel Standard Association in Illinois and Indiana. These counties serve as treatments in our study, and the remainder of the other counties serve as controls, with the exception of those counties that are contiguous to our treated counties. In the Synthetic Control Method, we use these control counties to create our synthetic units then we undertake a case by case study and analyze the treated counties individually. One of the outcomes used in this study is the total planted corn acres at the county level. We collected this data from the USDA NASS. We also use the yearly enrolled CRP acres at the county level as the other outcome; this data was collected from the Farm Service Agency (FSA).

Table 4.2 indicates counties among our treated counties that had an ethanol plant 
in operation before or after 2007. In the construction of our synthetic unit in the subsequent subsection, we use 2007 as our intervention year. In the literature, 2007 is often seen as the year when ethanol production started to pick up (Ifft et al., 2019; Li et al., 2019). Our first research question is trying to understand whether post-RFS mandate of 2007, total planted corn increased at the county level since corn serves as a feedstock in ethanol production. The second research issue is that after 2007, more land left the CRP program because of the RFS mandate. We focus on the CRP program since it provides environmental benefits that would be lost if land is converted back to commercial activities. Furthermore, the greenhouse gas (GHG) emission resulted from converting CRP land into cropland might reduce the GHG benefits gained from biofuels (Searchinger et al., 2008).

We begin with an investigation of the parallel trends of the outcomes used in this study and perform a simple Difference-in-Difference (DID) analysis at the state level. We apply this method to gain insights into how enrolled CRP and total planted corn acres reacted after the RFS of 2007 in Illinois and Indiana. We call these state effects in this study, "global effects". First, Figure 4.5 shows the time trends of the cumulative CRP enrollment and total planted corn acres for counties with an ethanol plant and counties without a plant in Illinois and Indiana. The comparison between the treated and control counties shows that between 2002 and 2007, cumulative enrolled CRP acres in Illinois were increasing in counties without a plant, however, in counties with a plant, we can see that the acres enrolled in the CRP were stagnant throughout this whole period. The total planted corn acres' trends during the same period also differ for counties with a plant and without a plant.

In Indiana, the cumulative enrolled CRP acres in counties without a plant were decreasing between 2002-2004, and started slightly increasing from 2004 up until 2007; the trend in counties with a plant seems to have stayed stagnant throughout the whole period. For total planted corn acres in Indiana, we can see that the trends show a similar pattern as those for the cumulative CRP graph, with counties without plants showing some fluctuation, and counties with a plant staying stagnant. Overall, these 
trend lines give us an overview of the state of enrolled CRP and total planted corn acreage in Illinois and Indiana. It also gives us a general understanding of whether ethanol plants were locating mainly in areas where corn production was booming, or whether they were implemented in areas where CRP enrollment is decreasing.

Second, to clarify the graphical implications mentioned above, we implement an OLS estimation with the following DID model, still at the state level:

$$
Y_{i, t}=\delta_{i}+\eta_{t}+\beta D_{i, t}+\mu_{i, t}
$$

Where $Y_{i, t}$ is the enrolled CRP acres or the total planted corn acres in county i and period t, $\delta_{i}$ is county fixed effects, $\eta_{t}$ is a common time effect, $\mu_{i, t}$ is an error term, and $D_{i, t}$ is a dummy variable that takes the value of one if a county has an ethanol plant in and after the intervention year 2007, and zero otherwise.

The same data used to obtain Figure 4.5 is used in this case to get the DID estimates in Table 4.1. The coefficients of the DID models in all the models are positive, suggesting that, after the RFS revision of 2007, more CRP land stayed in counties with an ethanol plant in Illinois and Indiana ${ }^{5}$, and these counties also saw their total planted corn acreage increase. Ifft et al. (2019) and Motamed et al. (2016) find similar results. Overall, these global effects at the state level do not give us much details, a part from informing us that after the RFS mandate of 2007, more land stayed in the CRP program, and total planted corn acres increased, hence no land use at the extensive margin. Since this study want to guide the local governments Of Indiana and Illinois in understanding areas that may be experiencing land use at the extensive margin, the global effects offered by the DID estimates might be misleading.

Furthermore, as explained earlier, the differences in the pre-intervention trajecto-

\footnotetext{
${ }^{5}$ Ifft et al. (2019) suggest interpreting these results this way, since during our study period there was a declining trend of CRP participation. Therefore, interpreting the positive estimates of the results as enrolled CRP acres increasing could be misleading, which is why we opt to interpret it as less land leaving the program.
} 
ries in Figure 4.5 for the treated counties and the control counties in some instances raise concerns. There may be unobserved predictors that differ between the comparison groups, and whose effects on enrolled CRP acres and the total planted corn changes over time. Although Figure 4.5 shows that the common parallel trend assumption might hold at some point in time, it would be better to explicitly control for possible differential trends for the counties with an ethanol plant.

In this study, we prefer the use of the Synthetic Control Method. This method has the applicability to advance the main goal of this paper, which is showing the impact of local ethanol production on enrolled CRP and total planted corn acres at the local level, while using the RFS expansion of 2007 as a natural experiment. This method is a data driven procedure that is suitable for comparative case studies (counties in Illinois and Indiana with an ethanol plant) that focus on the impact of a particular event or intervention (RFS expansion of 2007)(Abadie and Gardeazabal, 2003; Abadie et al., 2010; Abadie, 2021).

\subsubsection{Synthetic Control Analysis}

The Synthetic Control Method (SCM) is widely used in quasi-experimental studies that focus on the impact of a particular program or policy event. In practice, the SCM constructs a "counterfactual" synthetic control unit by weighting control-group counties such that the weighted average of outcomes and relevant covariates in the pre-RFS revision are close to their counterparts in the treated unit (Abadie and Gardeazabal, 2003; Abadie et al., 2010; Huang and Etienne, 2021; Ando, 2015; Kreif et al., 2016; Jakus and Akhundjanov, 2019). In this study, we follow the idea of Doudchenko and Imbens (2016) by including the lagged outcome variables as our predictors $(2000,2001,2002,2003,2004,2005,2006)$ - in this cited study, they inform us that including other covariates rarely mattered, however, in all our cases, we also include the size of the county and the CRP rental payments, as they all affect total planted corn and the cumulative acres enrolled in the CRP program (Table 4.6 and 
4.7 show the covariates balancing).

Following Kreif et al. (2016), Huang and Etienne (2021) and Jakus and Akhundjanov (2019), consider there exist $c+1$ of ethanol producing and non-ethanol producing counties in Illinois and Indiana where the first county $(c=1)$ is exposed to the RFS ethanol expansion mandate and the remaining counties $(c=2, \ldots, C+1)$ are not. In this suty, ethanol producing counties in Illinois and Indiana are our treated units, and non-ethanol counties are considered as the 'donor pool'. Outcomes are observed for T periods, and the policy of interest, the RFS mandate of 2007, starts in $T_{0}+1$. In this case, $1,2,3, \ldots T_{0}$ are the pre-intervention periods, and $T_{0}+1$, $T_{0}+2, T_{0}+3, \ldots . T$ are the post-treatment periods, where $1 \leq T_{o}<T$. The observed outcomes vector of each county are $Y_{c}=\left(Y_{c, 1} \ldots Y_{c, T 0} \ldots Y_{c, T}\right)^{\prime}$.

The observed enrolled CRP and total planted corn can be written as the sum of a treatment-free potential outcome, $Y_{c, t}^{N}$, and the effect of the treatment, $\sigma_{c, t}$, such that :

$$
Y_{c, t}=Y_{c, t}^{N}+\sigma_{c, t} D_{c, t}
$$

$$
Y_{c, t}^{N}=\alpha_{t}+\rho_{t} \mu_{c}+\beta_{t} Z_{c}+\epsilon_{i, t}
$$

Where $\alpha_{t}$ is a time fixed effect, $Z_{c}$ is a vector of time-invariant measured predictors with time varying coefficient vector $\beta_{t}, \mu_{c}$ are vector of time-invariant unobserved predictor variables with time-varying coefficients $\rho_{t}, D_{c, t}$ is an indicator variable that takes the value of 1 if a county is treated after 2007, and is 0 otherwise, $\epsilon_{c, t}$ are unobserved transitory shocks with zero mean. To some extent, the SCM method generalizes the DID method since one of the assumptions of the SCM is that the relationship between the outcome and the predictors is linear, therefore by allowing the 
effects of $\rho_{t}$ of the unobserved predictors $\mu_{c}$ to differ over time, the SCM differentiates itself from the DID method which constrains these effects to be constant (Kreif et al., 2016).

The objective is to estimate $\hat{\sigma}_{1, t}$, the effect of the treatment on enrolled CRP and total planted corn acres for the counties with an ethanol plant in the post RFS 2007 period, that is:

$$
\hat{\sigma}_{1, t}=Y_{1, t}-\hat{Y}_{1, t}^{N}
$$

for $t>T_{0}$. In this case, $\hat{Y}_{1, t}^{N}=\sum_{c=2}^{c+1} w_{c} Y_{c, t}$. Because $Y_{1 t}$ is observed, only $Y_{1 t}^{N}$ needs to be estimated to obtain $\hat{\sigma}_{1, t}$. A SCM unit is defined as a weighed average of the control counties in the donor pool (all other counties without an ethanol plant), and these weights are used to construct $Y_{1 t}^{N}$ (Abadie and Gardeazabal, 2003; Abadie et al., 2010, 2015). Therefore, a synthetic control unit can be represented by a $(\mathrm{C} \times 1)$ vector of weights $W=\left(w_{1}, \ldots, w_{c+1}\right)^{\prime}$ for counties $\mathrm{c}=2 \ldots, \mathrm{c}+1$, which satisfy $0<$ $w_{c}<1$ and $w_{2}+\ldots+w_{c+1}=1$. Using a vector of some optimal weights $W *=$ $\left(w *_{2}, \ldots, w *_{c+1}\right)^{\prime}, Y_{1 t}^{N}$ is estimated as the weighted average of $Y_{c, t}$.

In this study, $\hat{\sigma}_{1, t}$ is referred to as our post-effects. The statistical inference cannot be based on the magnitude of the post-effects only (Jakus and Akhundjanov, 2019). Instead, Galiani and Quistorff (2017) propose running placebo tests, i.e, running the same model on each untreated counties, assuming that they all had an ethanol plant in 2007 to get a distribution of in place placebo effects.In this exercise, one need not to include the treated units from being considered for the synthetic controls of these other units. Galiani and Quistorff (2017) also explain that if the placebo effects obtained are as large as the main estimate, then it is likely that estimated effect was observed by chance. Suppose that the estimated post-RFS 2007 effect for a particular post-treatment period is $\hat{\sigma}_{1, t}$ and that the corresponding placebos are $\hat{\sigma}_{1, t}^{P L}=\left(\hat{\sigma}_{1, t}^{P L}: c \neq\right.$ 1$.$) .$ 
Then, the two-sided p-value shown in Table 4.3 and 4.4 can be thought of as:

$$
p-\text { value }=\operatorname{Pr}\left(\left|\hat{\sigma}_{1, t}^{P L}\right| \geq\left|\hat{\sigma}_{1, t}\right|\right)=\left(\sum_{c \neq 1} 1\left(\left|\hat{\sigma}_{c, t}\right| \geq\left|\hat{\sigma}_{1, t}\right|\right)\right) / C
$$

We rely on the significance of the p-values for different post-treatment to assess whether Illinois and Indiana had cases of land use at the extensive margin between 2007 and 2017, the post RFS period. As Galiani and Quistorff (2017) suggest, when treatment is randomized, this becomes a classical case of randomization inferences ${ }^{6}$.

Since the SCM method does not allow for conventional parametric test of statistical significance level, we conduct one more diagnostic test of our results. In this exercise, we conduct a set of falsification tests as suggested in (Abadie et al., 2010, 2015). This exercise consists of taking some of the counties in the donor pool as placebos. To evaluate the size of the treatment effects, the ratio of the post-2007 root mean square prediction error $(\mathrm{RMSPE})^{7}$ to the pre-2007 RMSPE is used (Abadie et al., 2015). A larger pre/post treatment ratio suggests that there is a large gap between the actual and synthetic outcome in the post-treatment, which is indicative of a significant post treatment effect.

\subsection{Results}

\subsubsection{Post-Effects In Indiana and Illinois}

Table 4.3 and 4.4 show the post-effects or the SCM estimates and their p-values. In these tables, we only report counties that had statistically significant post-effects, since we wanted to understand those areas that had land use changes after the RFS mandate of 2007. For example, if we consider Macon, IL in 2010 (Table 4.3), we see that the gap in total planted corn between Macon and Synthetic Macon is around

\footnotetext{
${ }^{6}$ Randomization inference considers what would have happened under all possible random assignments, not just the one that happened to be selected for the selected experiment at hand.

${ }^{7}$ RMSPE measures the magnitude of the gap in the outcome variable of interest between each country and its synthetic counterpart.
} 
114696.1 acres $(\mathrm{p}$-value $=0.059)$. The $\mathrm{p}$-value in this case implies that the probability that the effects identified for Macon was due to a chance is 5.9 percent. In both Table 4.3 and 4.4, we can see that any p-value that was greater than 0.1 was associated to post-effects that were statistically not significant. As a result, during these years, we cannot assess the land use changes in acres, and hence detect land use at the extensive margin for the non-significant counties.

It is this reason that lead us in not presenting the results of counties ${ }^{8}$ that have p-values that are greater than 0.1 for all the post-effects years (2007-2017). For Indiana, these counties are Randolph, Grant, Putnam, Wabash, Davies and Posey; and for Illinois, Stephenson, Peoria, Henry, Putnam, Madison and Ford. Since we are dealing with multiple treated and multiple synthetic units in this study, we decide to focus on those counties in Indiana and Illinois that have statistically significant postRFS effects. These post-effects results highlight the various effects that exist when we analyze the localized effects of ethanol plant location on enrolled CRP and total planted corn acres by states. In Indiana for example, we can see that the counties with significant and non-significant post-effects all had ethanol plants starting operations in 2007 or after (Table 4.2). In Illinois, most of the counties that had significant post-effects also had ethanol plants that started operating around 2007. However, those counties that had non-significant results in Illinois seem to have had ethanol plants that started operating prior to 2007 as Table 4.2 shows.

There is a risk, however, that the post-effects found in Table 4.3 and 4.4 might not be plausible, questioning the effectiveness of the SCM method as an appropriate policy evaluation tool for the RFS mandate of 2007. Abadie (2021) suggest checking for anticipatory effects, that is SCM estimators may be biased if certain components of the intervention are put in place in advance of the formal implementation/enactment of the intervention. Since the Energy Act policy was amended several times before 2007, our post-effects might be prone to anticipatory effects. As a solution, Abadie (2021) suggest backdating the intervention in the data to a period before the antic-

\footnotetext{
${ }^{8}$ These results are available in the appendix
} 
ipatory effects. Following Abadie et al. (2015) and Abadie (2021), we conduct an "in-time placebo" test. In this exercise, we choose 2002 and 2005 as our intervention years, since the Energy Policy Act was amended in 2002 and 2005 before the Energy Independence and Security Act of 2007. In the cases where we choose 2002 as our intervention year to fix the anticipatory effects, we make this practical judgement since our data begin in 2000, and for the SCM to work one need a pre-intervention period, which is the year 2000 and 2001 in our case.

For example, Table 4.3 and 4.4 show that the post effects (outcome: enrolled CRP acres) for Ogle, IL, Tazewell, IL, Jasper, IN and Montgomery, IN all started being significant in 2007, the year of our intervention - as a consequence, these results indicate that enrolled CRP acres at the county level reacted in anticipation of the ethanol mandate of 2007 . In practice, it is at least preferable that the post-effects start being significant around 2008, one year after the intervention (Abadie, 2021). Therefore, in cases such as this one, one need to backdate the intervention year to capture the true post-effects.

Another problem that our post-effects results might be facing is the availability of a suitable control group. Abadie (2021) suggest eliminating the county with the highest weight in the donor pool in Figure 4.6 to assess whether our results considerably change. To address this, the leave out one selected donor county is used. This test aims to check if a county within the donor pool is driving our results. In constructing the synthetic unit, a county that has a higher weight than other donors can impact our results; we therefore drop this county and see whether our results still closely resemble our actual results.

We begin the next subsections by explaining the results for counties from Indiana that had significant post-effects, and then we move on to Illinois counties. In all the counties that had significant post effects, we assess the plausibility of these results (1) by addressing the anticipatory effects, and (2) by conducting a leave one out placebo test. 


\subsubsection{Jay County, IN (Outcome: Enrolled CRP Acres)}

We begin with the results for Jay, Indiana, with the enrolled CRP acres as the outcome. The dashed line indicates the synthetic Jay, and the solid line illustrates the actual path of Jay County, IN. In Figure 4.7, the graph for Jay County shows that Jay County and synthetic Jay track each other well until after 2005 when we start seeing a difference between the two lines. With the separation of the two lines happening before our intervention year, we perform the in-time placebo and the leave one out test as a sensitivity test of our results. Figure 4.6 shows the counties that served in the donor weight process while creating synthetic Jay. We can see that Marshall is the highest contributor. We therefore eliminate Marshall county from our sample and rerun the model. The leave one out panel in Figure 4.7 illustrates how the elimination of Marshall County did little in changing the path of both synthetic Jay and the actual Jay.

We then perform the in-time placebo test. The graph with 2002 as our intervention year has synthetic and actual Jay diverging around 2001, the year when the bill was introduced into Senate. Even though Table 4.4 shows positive and statistically significant effects post effects around 2016 and 2017, we find it hard to believe that these effects are caused by the RFS expansion from 2007. The post-treatment to pre-treatment RMSPE ratio test in Table 4.5 shows a weak evidence of significant treatment effects, since Jay is ranked 4th, when we compare the post/pre RMSPE ratio from the lowest to the highest. In this case, the separation between synthetic Jay and actual Jay in 2001 seems to imply that the bill's introduction in 2001 led landowners in Jay County to anticipate the Energy policy act of 2005 and its revision from 2007. 


\subsubsection{Jasper County, Indiana (Outcome: Enrolled CRP Acres and Total Planted Corn)}

Synthetic Jasper and actual Jasper (Figure 4.8) follow each other well in the pre2007 period. However, the separation between the two lines seems to have started around 2006. We conduct the leave one out test by taking out Pulaski, the highest donor weight from our sample, we find that the two graphs are quite similar, suggesting that Pulaski did not drive the results. When we conduct the in-time placebo test, with 2002 as our placebo intervention year, we still find that the separation between Jasper and its synthetic occurred in 2001. The post-treatment to pre-treatment RMSPE ratio in table 4.5 shows weak evidence of treatment effects since Jasper is ranked 5th of of 42 counties in terms low post/pre RMSPE ratios. Therefore, we conclude that the introduction of the bill in 2001 created some anticipatory effects in Jasper County. More land stayed in the CRP program because of the bill's introduction since Figure 4.8 shows that the hard line representing actual Jasper County kept increasing after 2001, the year where both synthetic Jasper and actual Jasper starts diverging (see in time placebo panel).

The results for Jasper County with total planted corn as the outcome (Figure 4.9) do show different findings compared to the enrolled CRP acres findings: (1) we find that the post-intervention to pre-intervention RMSPE ratio offer evidence of strong treatment effects, since Jasper, IN in this case has one of the largest post/pre RMSPE ratio in the whole pool. This is a sign that the RFS revision from 2007 had a large post effect on total planted corn in Jasper, Indiana.(2) Jasper and its synthetic seem to track each other well in the period prior to 2007.

When we perform the leave one out test, by eliminating Benton county, the highest donor, we find that unlike the original graph, the difference between Jasper and its synthetic started in 2007.(3) The post effects results in Table 4.4 are plausible since the ethanol plant in Jasper County started operating in 2007. We also conduct an in time placebo with 2005 as the intervention year. As the panel for in-time placebo 
shows in Figure 4.9, Jasper and synthetic Jasper follow the same path as the original graph in panel b. Furthermore, unlike, the results for the commutative CRP acres, we do not see any statistically significant post-effects in year 2007 in Table 4.4, which is indicative of no anticipatory effects - hence confirming the plausibility of the post 2007 RFS results. Looking at the gap between Jasper and Synthetic Jasper in panel b of Figure 4.9, we can see that more land got converted into corn production. In 2010, Table 4.4 shows that Jasper, IN started experiencing land use at the extensive margin 3 years after the RFS of 2007, since the post-effects are positive and statistically significant.

\subsubsection{Montgomery County, IN (Outcome: Enrolled CRP Acres) and Shelby County, IN (Outcome: Total Planted Corn)}

The results for Montgomery county (Figure 4.10) shows that Synthetic Montgomery and actual Montgomery track each other well prior to 2007, like Jay and Jasper counties, enrolled CRP cases in Montgomery County started diverging from its synthetic prior to 2007. We assess further these results by conducting a leave one out placebo test and find that Montgomery and its synthetic results were not impacted by Clark County, its highest donor. The in-time placebo test leads us to conclude that the enrolled CRP acres started adjusting around 2001, and the post-intervention to pre-intervention RMSPE ratio show weak evidence of treatment effects.

Shelby and Synthetic Shelby (Figure 4.11) do not track each other well prior to the RFS revision of 2007. Moreover, the leave one out test also performs poorly as well. However, the in-time placebo test does offer some interesting talking point. Prior to the placebo intervention year, synthetic Shelby and Shelby followed each other perfectly, and we see a sharp increase in total planted corn in 2002, highlighting the anticipatory effects from previous results. Nevertheless, these results illustrate a case where the donor pool performed poorly while creating the synthetic Shelby. In the next set of results, we present the results for the counties in the state of Illinois. 


\subsubsection{Ogle County, IL (Outcome: Enrolled CRP Acres)}

Ogle county and Synthetic Ogle (Figure 4.12) track each other perfectly prior to 2007, nonetheless the gap between both lines start prior to 2007. When we conduct the in-time placebo, the previous results that CRP land changed in anticipation of the RFS mandate hold in this case as well. Prior to 2002, Synthetic Ogle and Ogle were already diverging. The omission of Calhoun County from our sample, the highest donor, does improve the fit. Synthetic Ogle and actual Ogle now follow each other perfectly and the difference between the two lines happens at the intervention year. The large and significant post-treatment to pre-treatment RMSPE ratio (7.428) does lend credibility to the post effects values. Looking at the CRP enrollment trend graph in Ogle, IL, we see a consistent drop in CRP acreage. We conclude that this decrease in CRP land in Ogle County might be the result of the CRP land expiring, and it is not caused by the RFS expansion of $2007^{9}$.

\subsubsection{Tazewell County, IL (Outcome: Enrolled CRP Acres)}

The graphs for Tazewell County and synthetic Tazewell (Figure 4.13 track each other very well in terms of enrolled CRP acres, then actual Tazewell shows a jump in terms of CRP enrollment. This result means that more land stayed in the CRP post RFS. The in-time placebo test does suggests that Tazewell and its synthetic are sensitive to a change in time since we now observe a separation between Tazewell and Synthetic Tazewell that started in 2001. The leave one out test performed by omitting Christian County, the largest donor among the donor pool in creating synthetic Tazwell shows that Christian County was not driving our results in the first place. The post-treatment to pre-treatment RMSPE ratio is also large and infer significant treatment effects. Thus, Tazewell County had acres of the CRP land staying in the program, following the RFS mandate.

\footnotetext{
${ }^{9}$ for more on CRP land expiration, Hellerstein (2017) does a synthesis of the behavior of the CRP program in the past 20 years.
} 


\subsubsection{Macon and Crawford Counties, IL (Outcome: Total Planted Corn)}

The trajectories of Macon and its synthetic counterpart are shown in Figure 4.14. The levels and trends of total planted corn are fairly similar between Macon and synthetic Macon in the pre-RFS period, which suggests that the two units are fairly comparable in the post RFS period. Furthermore, the post-treatment to pre-treatment ratio (5.486) does support a large effect in the post-designation period. The gap between Macon and Synthetic Macon indicates that following the RFS mandate, total planted corn increased in Macon, IL (Figure 4.14). Table 4.3 shows that the positive post effects had turned negative by year 2012 and 2014 -indicating some fluctuating in the long-term effects of the RFS mandate of 2007. We also conduct an in time placebo (panel b Figure 4.14) with 2005 as the intervention year, and find that Macon and Synthetic Macon follow the same path as the original graph in panel a (see Figure 4.14).

Finally, Figure 4.15 shows that Crawford and Synthetic Crawford follow two different paths in the pre-designation period, which does not lend any credibility to the post-effects results from Table 4.3.

\subsection{Discussion}

Of the 20 counties that had an ethanol plant in Illinois and Indiana, only 8 had significant post-effects, and among these 8 treated units, the plausibility of these post-effects was only found in only 4 cases. Given the importance of the ethanol expansion in recent political debates, we decide to dig deeper into our statistically significant cases to try to understand whether the Energy Independence Act of 2007 led to land-use changes in ethanol-producing counties in Illinois and Indiana. Our discussion begins with the anticipatory effects that we often find in cases with enrolled CRP acres as the outcome. We then discuss cases that had total planted corn acres 
significant in the post-effects.

Many policies are implemented with a significant lag from their enactment date. Such events are described as anticipatory effects in the causal effect's econometrics literature (Alpert, 2016). In our case, the Energy Policy Act was introduced toward the end of 2001 and signed into law in 2005, and subsequently revised in 2007. Many studies that have analyzed the impact of local ethanol production on land-use change have looked at land-use changes after the RFS revision of 2007 in larger geographical units (Ifft et al., 2019; Motamed et al., 2016; Chen and Khanna, 2018; Wright and Wimberly, 2013). In this study, we consistently find that changes in the enrolled CRP acres happened way before 2007 when we look at this issue at the county level. Furthermore, ethanol plants in Illinois started operating before 2007, whereas plants in Indiana started around 2007, the year when the RFS revision happened.

Looking at the effects of ethanol plant locations on land-use changes at the state level can be misleading. For example, the global effects of our Difference-in-Difference model show that in both Indiana and Illinois, enrolled CRP and total planted corn acres increased after 2007. Nonetheless, as we just saw in the previous paragraph, Indiana and Illinois started producing ethanol at different periods in time. The effects of this time lag in starting operation dates between Indiana and Illinois can only be found when we analyze local effects. For example, in Ogle, IL, and Tazewell, IL, we can see that despite backdating our intervention year to 2002 to avoid the anticipatory effects as advised by Abadie (2021), we still find that the gap between these counties and their synthetic counties happened around 2001. In all these counties, the posteffects vary, with some years showing that land stayed in the CRP program, and other years showing that the CRP program lost land after the RFS of 2007 . We can see that the variation in the results from one case to another is indicative of strong local effects. Therefore, understanding the effects of local ethanol production needs to be conducted at smaller geographical scales, since this strategy provides a more comprehensive analysis compared to studies that look at this issue at larger geographical scales. 
We also find plausible post-effects for Jasper, IN, and Macon, IL when we use total planted corn acres as the outcome. In these two cases, we find that the posteffects happened after 2007. Contrary to the results for the enrolled CRP, the total planted corn results do not have post-effects (see Table 4.3 and 4.4) that start being significant in the year 2007, the intervention year - this is a sign that there are no anticipatory effects issues in these results. In the previous section, we only performed the anticipatory effects placebo tests because we were relying on the graphical results of these two counties. The fact that Table 4.3 and 4.4 indicates anticipatory effects for the enrolled CRP acres outcome only, shows that CRP administrators may have anticipated the RFS policies ahead of time. The literature on the CRP enrollment trends does suggest that the CRP program changes enrollment mechanism to keep land under conservation competitive after the enactment of policies that can change CRP enrollments (Hellerstein, 2017; Ifft et al., 2019).

In the end, we find that studying the impact of ethanol plants on land-use changes while using the SCM helps us understand the spatial and temporal post-effects of the RFS mandate of 2007. For both total planted corn acres and enrolled CRP acres, we find that after the RFS expansion of 2007, the county-level SCM estimates varied by the post-intervention years and by counties. In some years, we have cases of land use at the extensive margin, e.g., Macon, IL, and Jasper, IN ( see Table 4.3 and 4.4 post effects for total planted corn: 2010), and in other cases, we have no evidence of land use at the extensive margin because the gap between the synthetic counties and their counties counterparts is negative, e.g., Macon, IL (Table 4.3 post-effects for total planted corn: 2012 and 2014). In other years, we also find that more land stayed in the CRP program due to the changes in the mechanisms that this program received over time (see Table 4.3 for Tazewell, IL results). However, we also see cases where land left the CRP program (see Table 4.3 for Ogle, IL results). Overall, taking into account that the results can vary by ethanol plant was the key in finding these geographical and temporal heterogeneous effects. 


\subsection{Conclusion}

Previous studies that have analyzed the impact of ethanol plants on land-use changes have mainly provided point estimates of these impacts at larger geographical units (Motamed et al., 2016; Ifft et al., 2019; Li et al., 2019). However, ethanol production is an activity that takes place at smaller geographical units, such as counties as Figure 4.3 and 4.4 show. In this study, we use the Synthetic Control Method (SCM) of Abadie et al. (2010, 2015); Abadie (2021) to understand the post-effects of the RFS mandate of 2007 in counties in Illinois and Indiana that had an ethanol plant on enrolled CRP and total planted corn acres. This method is preferable since it relaxes the parallel trends assumption of the Difference-in Difference method that was violated in similar studies (Ifft et al., 2019; Arora et al., 2016), and it also creates counterfactual outcomes for the counties with an ethanol plant (treated) from the outcomes of the counties without an ethanol plant (controls) by weighting the outcome of these control units. Finally, this method offers post-effects estimates for each county that had an ethanol plant in Illinois and Indiana.

The results from this analysis show spatial and dynamic heterogeneous post-effects when we analyze the local effects of ethanol production on land-use change on a caseby-case basis. For example, we find that after 2007 different counties in Illinois and Indiana had land that stayed in the CRP program and land that left the program on the one hand. These post RFS expansion effects seem to have manifested themselves right after 2007 in some cases, but we also see some long-term effects as is the case for Ogle, IL in Table 4.3 for example. In this case, we see that Ogle, IL was still experiencing the post-RFS effects of 2007 up until 2017.

On the other hand, the effects on total planted corn acres seem to follow the same geographical and temporal effects. Depending on a county, post RFS expansion effects were both positive and negative in the years following the RFS expansion in Illinois and Indiana. For example, Macon, IL shows some significant positive and negative post-effects in Table 4.3, and Jasper, IN in Table 4.4 show positive post-effects that 
started in 2010. Overall, we see that for both Illinois and Indiana, the SCM estimates vary by year and by county, and they can manifest themselves after a long period after the RFS of 2007.

One shortcoming of this study is the anticipatory effects that we often see in our graphical analysis of the synthetic analysis units for cases with enrolled CRP as the outcome. We argue in this study that this can be caused by two factors. First, the RFS mandates received several amendments before 2007. Hence, in some graphical analyses, the post-effects could be picking up some of these previous revisions. It was important to backdate the intervention year to 2002 and 2005 to assess the plausibility of our results. We find, for example, that our post-effects outcomes were impacted by changes in the mechanism of the CRP that this program received around the time of the RFS mandates for those cases that had land staying in the CRP program. In the cases where land was leaving the CRP program, it could be that the post-effects were picking up the general trends in CRP enrollment, which was decreasing in general after 2007 (Hellerstein, 2017; Ifft et al., 2019).

Second, ethanol plants started operating in the 1970 's in Illinois, whereas the first ethanol plant in Indiana started operating sometime in 2007. This difference in the start of operation could be why our results indicate that most of the counties that showed non-significant post-effects in Illinois had their ethanol plants operation starting before 2007. It could also be why our results for Jasper, IN show, that the gap in Jasper, IN and synthetic Jasper, IN for total planted corn as the outcome started diverging around 2005, when we backdated our intervention year to 2005 to avoid the anticipatory effects.

To conclude, we see in this study that studying the post-effects of policies such as the RFS of 2007 at the local level offers detailed information that one cannot access if we rely on single-point estimates such as those found in Table 4.1. To understand, the local impacts of ethanol production on land-use changes, this study demonstrates how getting the effects at the county level is essential to get the full picture. These results clearly show that post-RFS effects vary by case and by post-intervention years. 
We believe that the results of our study are suited for local land use policymakers that want to address issues of land use at the extensive margin, since these results provide comprehensive localized effects of local ethanol production on enrolled CRP acres and total planted corn acres. 


\subsection{Figures and Tables}

Figure 4.1: Production Volume of Fuel Ethanol in the United States 2000-2020 (in Million Gallons)

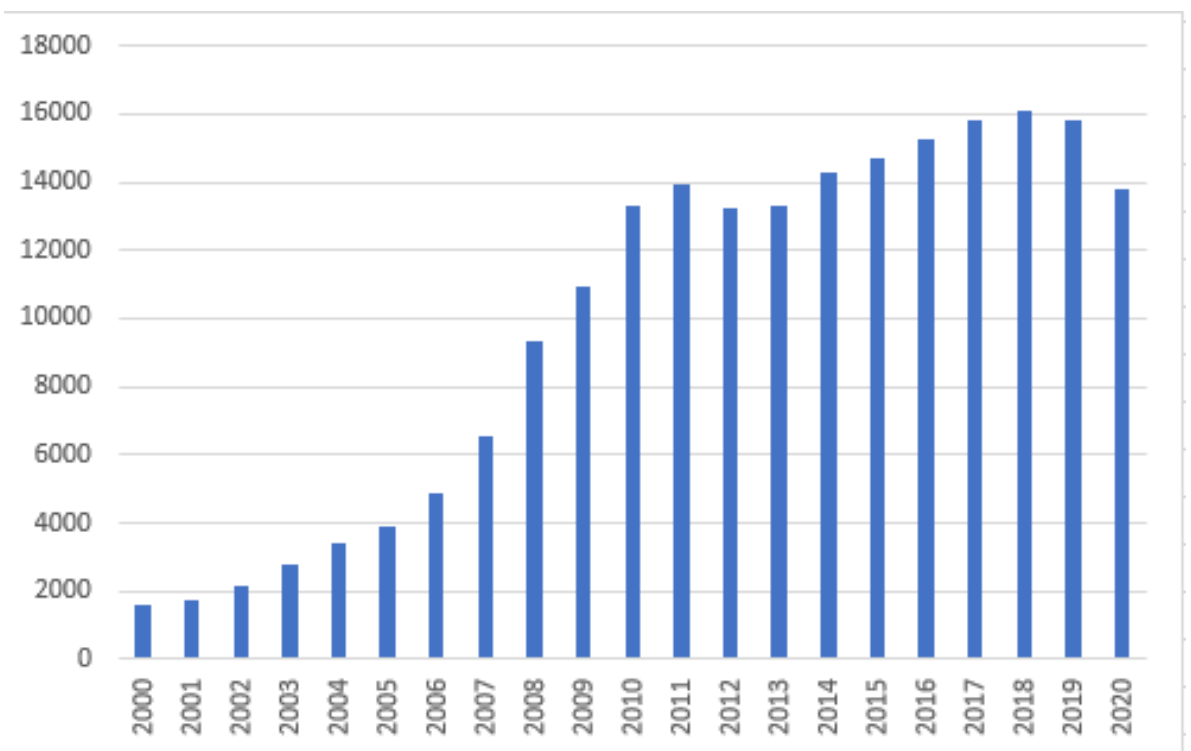

Notes: Sources (Sönnichsen, 2020) 
Figure 4.2: Ethanol Producing States

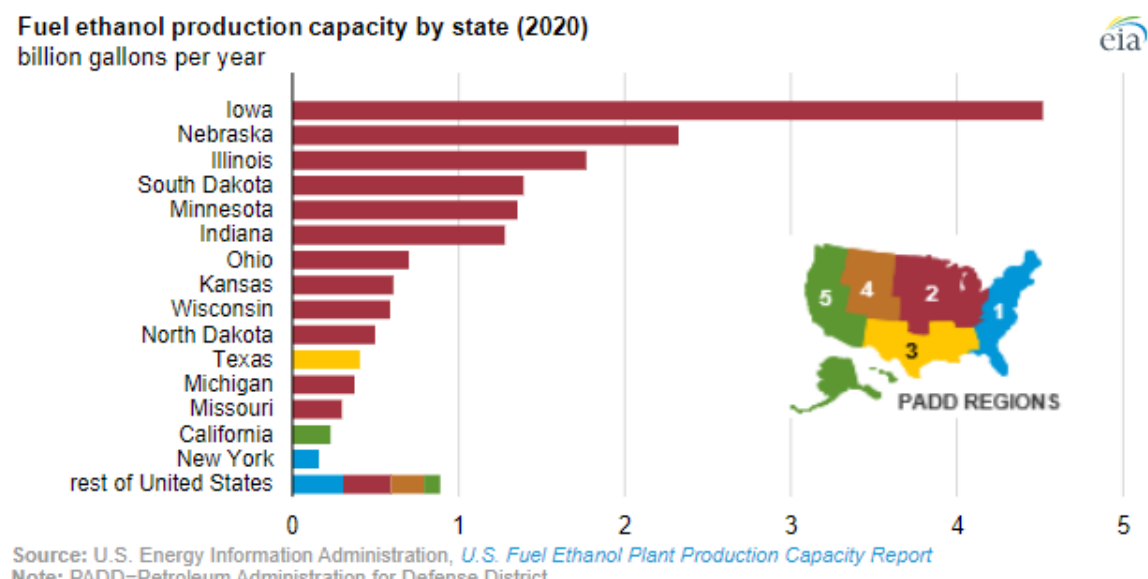


Table 4.1: Difference In Difference at the State Level

\begin{tabular}{|l|c|c|c|l|}
\hline & $\begin{array}{l}\text { DID for } \\
\text { CRP Illinois }\end{array}$ & $\begin{array}{l}\text { DID for } \\
\text { CRP Indi- } \\
\text { ana }\end{array}$ & $\begin{array}{l}\text { DID for } \\
\text { Corn Acres } \\
\text { Illinois }\end{array}$ & $\begin{array}{l}\text { DID for } \\
\text { Corn Acres } \\
\text { Indiana }\end{array}$ \\
\hline $\begin{array}{l}\text { DID Esti- } \\
\text { mates }\end{array}$ & $954.791^{* * *}$ & $614.178^{* * *}$ & $4372.751^{* * *}$ & $2596.692^{* * *}$ \\
\hline R-squared & 0.056 & 0.067 & 0.011 & 0.02 \\
\hline Observations & 1836 & 1656 & 1776 & 1545 \\
\hline $\begin{array}{l}\text { Treated } \\
\text { units }\end{array}$ & 10 & 10 & 10 & 10 \\
\hline
\end{tabular}

Statistical significance: "*" 10 percent, $" * * " 5$ percent, $" * * * " 1$ percent. $p$-value in parentheses. 
Table 4.2: Ethanol Plants Starting Operation Year

\begin{tabular}{|c|c|c|c|}
\hline $\begin{array}{l}\text { Treated } \\
\text { counties } \\
\text { in Illinois }\end{array}$ & $\begin{array}{l}\text { Year's ethanol } \\
\text { plant started } \\
\text { operating }\end{array}$ & $\begin{array}{l}\text { Treated counties in } \\
\text { Indiana }\end{array}$ & $\begin{array}{l}\text { Year's ethanol } \\
\text { plant started oper- } \\
\text { ating }\end{array}$ \\
\hline Stephenson & Before to 2007 & Randolph & After 2007 \\
\hline Tazewell & After 2007 & Grant & After 2007 \\
\hline Macon & After 2007 & Daviess & After 2007 \\
\hline Peoria & Before 2007 & Posey & After 2007 \\
\hline Henry & After 2007 & Jasper & 2007 \\
\hline Crawford & Before 2007 & Montgomery & - \\
\hline Ogle & 2007 & Putnam & After 2007 \\
\hline Putnam & Before 2007 & Wabash & After 2007 \\
\hline Madison & Before 2007 & Jay & After 2007 \\
\hline Ford & 2005 & Shelby & After 2007 \\
\hline
\end{tabular}

Notes: The list of ethanol plants, their locations and companies names was obtained from the Renewable Fuel Association (RFA). After visiting the companies websites to determine their starting date, some of the companies did not have this information. This explains why some starting dates are unknown in this table. 
Figure 4.3: Treated Counties in Illinois

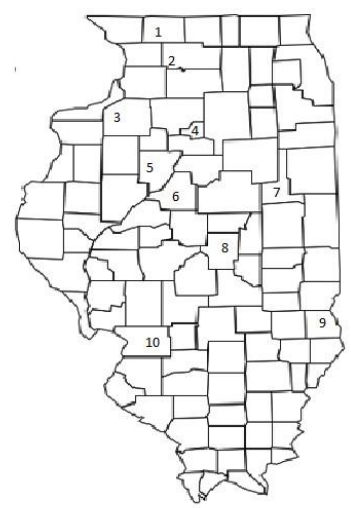

County names: 1.Stephenson 2.Ogle 3.Henry 4.Putnam 5.Peoria 6.Tazewell 7.Ford 8.Macon 9.Crawford 10. Madison

Figure 4.4: Treated Counties in Indiana

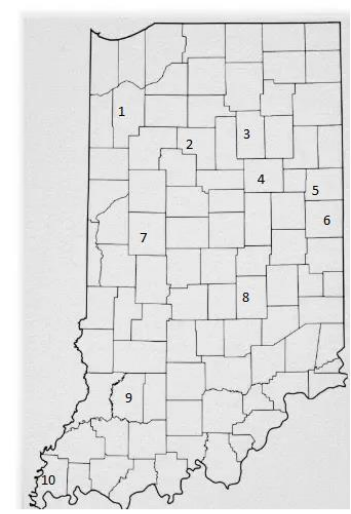


Figure 4.5: Parallel Trends for Illinois and Indiana

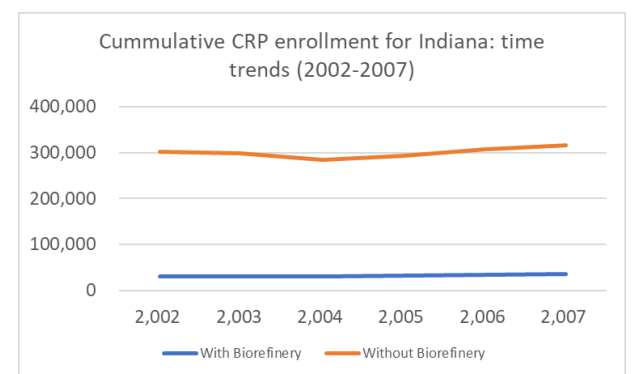

(a) Enrolled CRP Acres in Indiana (2002-2007)

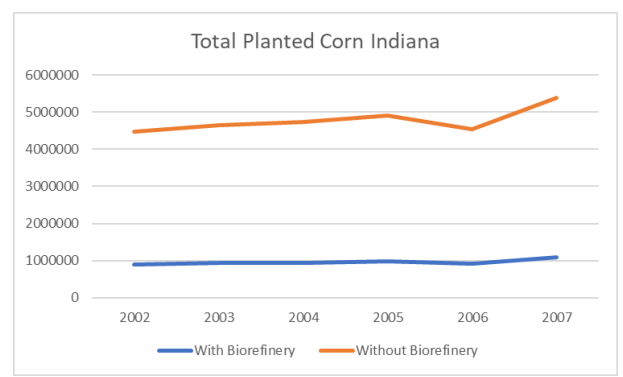

(c) Total Planted Corn in Indiana (2002- (d) Total Planted Corn in Illinois (20022007)

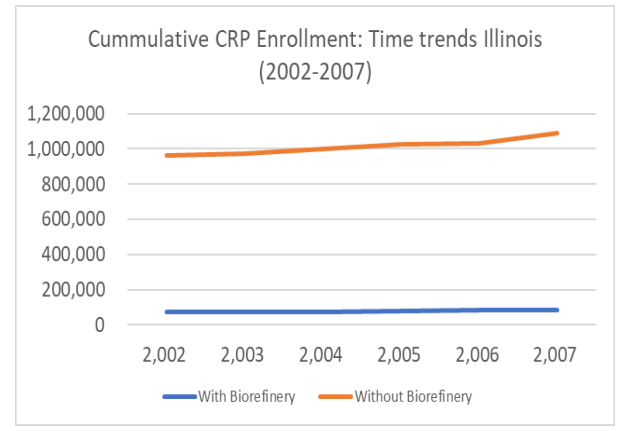

(b) Enrolled CRP Acres in Illinois (20022007)

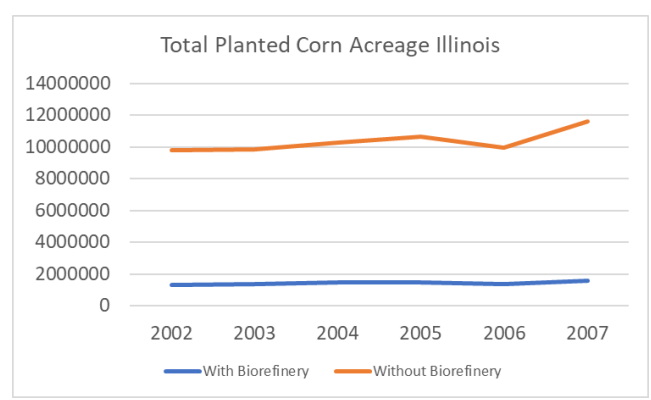
2007) 
Figure 4.6: Donor Weights

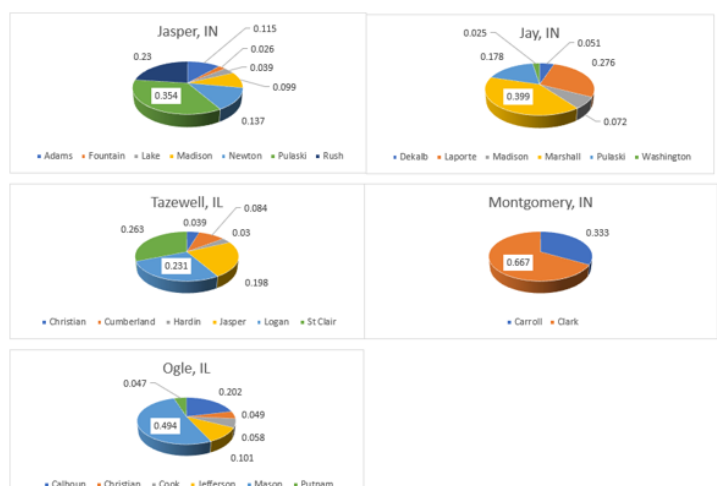

(a) Donor Weights for cumulative CRP acres as the outcome

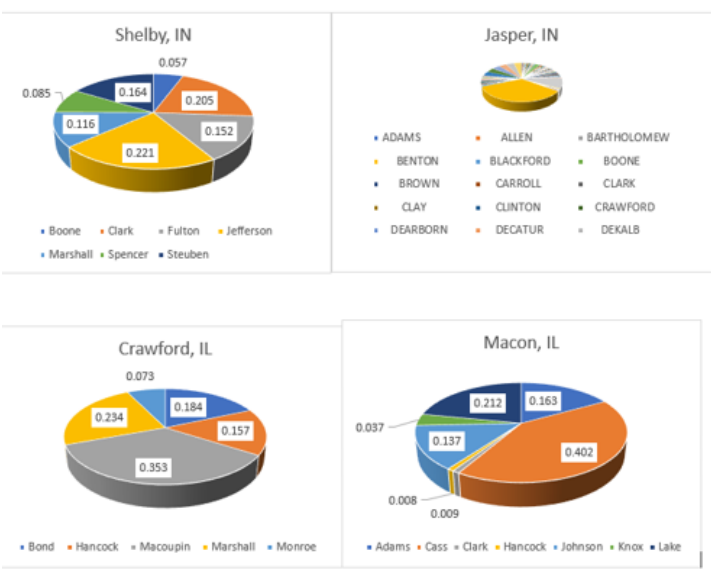

(b) Donor Weights for total planted corn acreage as the outcome 
Table 4.3: Post-treatment Effects for Statistically Significant Counties in Illinois

\begin{tabular}{|c|c|c|c|c|}
\hline & $\begin{array}{l}\text { Cumulative } \\
\text { CRP en- } \\
\text { rollment: } \\
\text { Ogle } \\
\text { (Acres) }\end{array}$ & $\begin{array}{l}\text { Cumulative } \\
\text { CRP en- } \\
\text { rollment: } \\
\text { Tazewell } \\
\text { (Acres) }\end{array}$ & $\begin{array}{l}\text { Total } \\
\text { Planted } \\
\text { Corn: } \\
\text { Macon } \\
\text { (Acres) }\end{array}$ & $\begin{array}{l}\text { Total } \\
\text { Planted } \\
\text { Corn: } \\
\text { Crawford } \\
\text { (Acres) }\end{array}$ \\
\hline 2007 & $\begin{array}{c}-879.895^{*} \\
(0.099)\end{array}$ & $\begin{array}{c}942.124^{*} \\
(0.069)\end{array}$ & $\begin{array}{c}-13547.7 \\
(0.554)\end{array}$ & $\begin{array}{c}100069.5 \\
(0.596)\end{array}$ \\
\hline 2008 & $\begin{array}{c}-1136.319 \\
(0.128) \\
\end{array}$ & $\begin{array}{c}590.073 \\
(0.366) \\
\end{array}$ & $\begin{array}{c}-20553.4 \\
(0.386) \\
\end{array}$ & $\begin{array}{c}85504 \\
(0.138) \\
\end{array}$ \\
\hline 2009 & $\begin{array}{c}-1542.245^{*} \\
(0.099)\end{array}$ & $\begin{array}{c}877.759 \\
(0.306)\end{array}$ & $\begin{array}{c}30888.5 \\
(0.257)\end{array}$ & $\begin{array}{c}-16458.5 \\
(0.396)\end{array}$ \\
\hline 2010 & $\begin{array}{c}-2156.239 * * \\
(0.049)\end{array}$ & $\begin{array}{c}1140.168 \\
(0.267) \\
\end{array}$ & $\begin{array}{c}114696.1^{*} \\
(0.059)\end{array}$ & $\begin{array}{c}-994 \\
(0.94) \\
\end{array}$ \\
\hline 2011 & $\begin{array}{c}-3394.749^{* *} \\
(0.039)\end{array}$ & $\begin{array}{c}1068.099 \\
(0.356)\end{array}$ & $\begin{array}{c}-33842.2 \\
(0.287)\end{array}$ & $\begin{array}{l}12747.5 \\
(0.544)\end{array}$ \\
\hline 2012 & $\begin{array}{c}-3380.844^{*} \\
(0.059)\end{array}$ & $\begin{array}{c}1116.153 \\
(0.445)\end{array}$ & $\begin{array}{c}-107934.9^{*} \\
(0.079)\end{array}$ & $\begin{array}{l}-41168 \\
(0.277) \\
\end{array}$ \\
\hline 2013 & $\begin{array}{c}-3935.422^{*} \\
(0.079)\end{array}$ & $\begin{array}{c}1259.916 \\
(0.445)\end{array}$ & $\begin{array}{c}87815.4 \\
(0.118)\end{array}$ & $\begin{array}{c}-61818.5 \\
(0.188)\end{array}$ \\
\hline 2014 & $\begin{array}{c}-4060.557^{*} \\
(0.069)\end{array}$ & $\begin{array}{c}1383.088 \\
(0.425)\end{array}$ & $\begin{array}{c}-92776.7^{* *} \\
(0.049)\end{array}$ & $\begin{array}{c}-88915.5^{*} \\
(0.069)\end{array}$ \\
\hline 2015 & $\begin{array}{c}-5129.032^{* *} \\
(0.039)\end{array}$ & $\begin{array}{c}1384.694 \\
(0.425)\end{array}$ & $\begin{array}{l}-6635.5 \\
(0.712)\end{array}$ & $\begin{array}{l}-50226 \\
(0.207)\end{array}$ \\
\hline 2016 & $\begin{array}{c}-6511.218^{* *} \\
(0.029)\end{array}$ & $\begin{array}{c}1608.98 \\
(0.475)\end{array}$ & $\begin{array}{c}-21689.4 \\
(0.396)\end{array}$ & $\begin{array}{c}19756 \\
(0.425)\end{array}$ \\
\hline 2017 & $\begin{array}{c}-7126.891^{* *} \\
(0.039)\end{array}$ & $\begin{array}{c}1645.609 \\
(0.495)\end{array}$ & $\begin{array}{c}-12272.7 \\
(0.584)\end{array}$ & $\begin{array}{c}96774.5^{*} \\
(0.059)\end{array}$ \\
\hline
\end{tabular}

Notes: Statistical significance: "*" 10 percent, "**” 5 percent, "***” 1 percent. p-value in parentheses. These p-values are obtained by using the two-sided test from (Galiani and Quistorff, 2017) 
Table 4.4: Post-treatment Effects for Statistically Significant Counties In Indiana

\begin{tabular}{|c|c|c|c|c|c|}
\hline & $\begin{array}{l}\text { Cumulative } \\
\text { CRP en- } \\
\text { rollment: } \\
\text { Jasper } \\
\text { (Acres) }\end{array}$ & $\begin{array}{l}\text { Cumulative } \\
\text { CRP en- } \\
\text { rollment: } \\
\text { Jay } \\
\text { (Acres): }\end{array}$ & $\begin{array}{l}\text { Cumulative } \\
\text { CRP en- } \\
\text { rollment: } \\
\text { Montgomer } \\
\text { (Acres): }\end{array}$ & $\begin{array}{l}\text { Total } \\
\text { Planted } \\
\text { Corn } \\
\text { Shelby } \\
\text { (Acres): }\end{array}$ & $\begin{array}{l}\text { Total } \\
\text { Planted } \\
\text { Corn } \\
\text { Jasper } \\
\text { (Acres): }\end{array}$ \\
\hline 2007 & $\begin{array}{c}-338.811^{*} \\
(0.0989)\end{array}$ & $\begin{array}{c}-222.826 \\
(0.1978)\end{array}$ & $\begin{array}{c}379.964^{*} \\
(0.065)\end{array}$ & $\begin{array}{c}-4432.4 \\
(0.472)\end{array}$ & $\begin{array}{c}-4186.6 \\
(0.494)\end{array}$ \\
\hline 2008 & $\begin{array}{c}-394.033 \\
(0.153)\end{array}$ & $\begin{array}{r}-346.698 \\
(0.1755)\end{array}$ & $\begin{array}{l}477.607 \\
(0.1098)\end{array}$ & $\begin{array}{r}-5689.7 \\
(0.395)\end{array}$ & $\begin{array}{c}-11764.3 \\
(0.175)\end{array}$ \\
\hline 2009 & $\begin{array}{l}-271.472 \\
(0.3186)\end{array}$ & $\begin{array}{c}-11.7067 \\
(0.978)\end{array}$ & $\begin{array}{l}362.427 \\
(0.2747)\end{array}$ & $\begin{array}{l}1472.2 \\
(0.769)\end{array}$ & $\begin{array}{c}-15014.7 \\
(0.237)\end{array}$ \\
\hline 2010 & $\begin{array}{l}-46.693 \\
(0.8131)\end{array}$ & $\begin{array}{l}4.4243 \\
(0.967)\end{array}$ & $\begin{array}{c}701.209^{*} \\
(0.0989)\end{array}$ & $\begin{array}{r}-7684.3 \\
(0.362)\end{array}$ & $\begin{array}{c}73188.2^{* *} \\
(0.032)\end{array}$ \\
\hline 2011 & $\begin{array}{c}-325.651 \\
(0.296) \\
\end{array}$ & $\begin{array}{l}133.903 \\
(0.6373)\end{array}$ & $\begin{array}{c}819.936^{*} \\
(0.0879)\end{array}$ & $\begin{array}{l}1101.8 \\
(0.824)\end{array}$ & $\begin{array}{c}73541.3^{* *} \\
(0.032)\end{array}$ \\
\hline 2012 & $\begin{array}{c}-564.366 \\
(0.142)\end{array}$ & $\begin{array}{c}280.733 \\
(0.4395)\end{array}$ & $\begin{array}{c}1151.42^{* *} \\
(0.0549)\end{array}$ & $\begin{array}{c}-60455.8^{* *} \\
(0.054)\end{array}$ & $\begin{array}{l}57715^{*} \\
(0.065)\end{array}$ \\
\hline 2013 & $\begin{array}{c}-831.796^{*} \\
(0.0989)\end{array}$ & $\begin{array}{l}534.151 \\
(0.1758)\end{array}$ & $\begin{array}{c}1328.582^{*} \\
(0.0659)\end{array}$ & $\begin{array}{c}-60440.3^{*} \\
(0.065)\end{array}$ & $\begin{array}{c}73545.6^{* *} \\
(0.021)\end{array}$ \\
\hline 2014 & $\begin{array}{r}-196.691 \\
(0.6153)\end{array}$ & $\begin{array}{c}671.236 \\
(0.1428)\end{array}$ & $\begin{array}{c}1386.574^{*} \\
(0.0769)\end{array}$ & $\begin{array}{c}-60449.4 \\
(0.065)\end{array}$ & $\begin{array}{c}78174.7^{* * *} \\
(0.01)\end{array}$ \\
\hline 2015 & $\begin{array}{c}-51.709 \\
(0.912)\end{array}$ & $\begin{array}{l}828.669 \\
(0.1422)\end{array}$ & $\begin{array}{c}1529.854^{* *} \\
(0.0329)\end{array}$ & $\begin{array}{c}-60426.2^{*} \\
(0.087)\end{array}$ & $\begin{array}{c}82192.9^{* *} \\
(0.021)\end{array}$ \\
\hline 2016 & $\begin{array}{l}39.854 \\
(0.978)\end{array}$ & $\begin{array}{c}1770.114^{* *} \\
(0.0329)\end{array}$ & $\begin{array}{c}1589.46^{*} \\
(0.0659)\end{array}$ & $\begin{array}{c}-60103.5 \\
(0.109)\end{array}$ & $\begin{array}{c}62969.7^{*} \\
(0.065)\end{array}$ \\
\hline 2017 & $\begin{array}{c}508.247 \\
(0.318)\end{array}$ & $\begin{array}{c}1912.812^{* *} \\
(0.0439)\end{array}$ & $\begin{array}{c}1850.318^{* *} \\
(0.0549)\end{array}$ & $\begin{array}{c}-53357.2 \\
(0.142)\end{array}$ & $\begin{array}{c}86963.2^{*} \\
(0.054)\end{array}$ \\
\hline
\end{tabular}

Notes: Statistical significance: "*" 10 percent, "**" 5 percent, "***" 1 percent. p-value in parentheses. These p-values are obtained by using the two-sided test from (Galiani and Quistorff, 2017) 
Table 4.5: Post/Pre RMSPE Ratio

\begin{tabular}{l|l|l}
\hline Counties & $\begin{array}{l}\text { Post/Pre } \\
\text { RMSPE } \\
\text { Ratios }\end{array}$ & Units Ranks and Percentages \\
\hline Jay, IN (enrolled CRP acres) & 3.208 & $4 / 27$ and 14 percent \\
\hline Jasper, IN (enrolled CRP acres) & 3.2 & $5 / 42$ and 11 percent \\
\hline Montgomery, IN (enrolled CRP acres) & 3.198 & $9 / 36$ and 25 percent \\
\hline Ogle. IL (enrolled CRP acres) & 7.428 & $42 / 48$ and 87 percent \\
\hline Tazewell. IL(enrolled CRP acres) & 7.442 & $32 / 40$ and 80 percent \\
\hline Shelby, IN (total planted corn) & 9.34 & $6 / 36$ and 16 percent \\
\hline Jasper, IN (total planted corn) & 9.56 & $44 / 48$ and 91 percent \\
\hline Crawford, IL (total planted corn) & 5.324 & $4 / 36$ and 11 percent \\
\hline Macon, IL (total planted corn) & 5.486 & $25 / 29$ and 86 percent \\
\hline
\end{tabular}

Notes: The RMSPE tests are based on placebo units with pre-RMSE 2007 that are less or equal to twice the preRMSE of each county with an ethanol plant that had post-effects that were statistically significant.Percentage is a proxy for p-value, which is obtained from the ratio of the treatment unit rank to the number of all units (Abadie et al., 2015). 
Table 4.6: Predictor Balance with Planted Corn Acres as the Outcome

\begin{tabular}{|c|c|c|c|c|c|c|c|c|}
\hline & Shelby, IN & & Jasper, IN & & Crawford, IL & & Macon, IL & \\
\hline & Treated & Synthetic & Treated & Synthetic & Treated & Synthetic & Treated & Synthetic \\
\hline \begin{tabular}{|l|} 
Planted \\
corn \\
acreage \\
$(2000)$
\end{tabular} & 70200 & 70490.6 & 37800 & 37455.6 & 111500 & 169654.4 & 78000 & 81900 \\
\hline \begin{tabular}{|l} 
Planted \\
corn \\
acreage \\
$(2001)$
\end{tabular} & 79900 & 79064 & 42000 & 41630.1 & 95000 & 139761 & 151000 & 150832 \\
\hline $\begin{array}{l}\text { Planted } \\
\text { corn } \\
\text { acreage } \\
(2002)\end{array}$ & 66200 & 68088 & 42000 & 41696.2 & 147000 & 134110 & 195000 & 193785.5 \\
\hline $\begin{array}{l}\text { Planted } \\
\text { corn } \\
\text { acreage } \\
(2003)\end{array}$ & 87000 & 75786.4 & 44800 & 44492.4 & 132000 & 112471.4 & 93000 & 92932.1 \\
\hline $\begin{array}{l}\text { Planted } \\
\text { corn } \\
\text { acreage } \\
(2004)\end{array}$ & 67600 & 71072.6 & 41000 & 40641.8 & 279000 & 219613.9 & 119000 & 117878.7 \\
\hline $\begin{array}{l}\text { Planted } \\
\text { corn } \\
\text { acreage } \\
(2005)\end{array}$ & 56800 & 67944.5 & 45000 & 44660.3 & 150000 & 140877.2 & 110000 & 109603.3 \\
\hline $\begin{array}{l}\text { Planted } \\
\text { corn } \\
\text { acreage } \\
(2006)\end{array}$ & 78500 & 73568.9 & 38500 & 38145 & 346000 & 266074 & 81000 & 81914.9 \\
\hline $\begin{array}{l}\text { County } \\
\text { sizes }\end{array}$ & 411.15 & 380.14 & 559.2 & 404.95 & 443 & 496.1 & 572 & 491.995 \\
\hline $\begin{array}{l}\text { CRP } \\
\text { rental } \\
\text { rates }\end{array}$ & 31472.18 & 359594.3 & 341116.5 & 323108.7 & 1242525 & 981810.2 & 350612.8 & 1072914 \\
\hline
\end{tabular}


Table 4.7: Predictor Balance with Cumulative CRP Acres as the Outcome

\begin{tabular}{|c|c|c|c|c|c|c|c|c|c|c|}
\hline & Jay, IN & & Jasper, IN & & Montgomery, IN & & Ogle, IL & & Tazewell, IL & \\
\hline & Treated & Synthetic & Treated & Synthetic & Treated & Synthetic & Treated & Synthetic & Treated & Synthetic \\
\hline $\begin{array}{l}\text { Cumulative } \\
\text { CRP } \\
\text { acres } \\
(2000)\end{array}$ & 5000.4 & 5063.84 & 2437 & 2443.246 & 1374 & 1429.196 & 9703.6 & 9709.849 & 4606.2 & 4621.715 \\
\hline $\begin{array}{l}\text { Cumulative } \\
\text { CRP } \\
\text { acres } \\
(2001)\end{array}$ & 5712.1 & 5674.751 & 2980.2 & 2976.646 & 1990.2 & 2089.479 & 11163.5 & 11168.66 & 6688.5 & 6690.129 \\
\hline $\begin{array}{l}\text { Cumulative } \\
\text { CRP } \\
\text { acres } \\
(2002)\end{array}$ & 5808.3 & 5856.887 & 3478.8 & 3484.962 & 2338.1 & 2470.759 & 11530.6 & 11491.63 & 7639.4 & 7575.263 \\
\hline $\begin{array}{l}\text { Cumulative } \\
\text { CRP } \\
\text { acres } \\
(2003)\end{array}$ & 5924.4 & 5874.416 & 3628.2 & 3617.304 & 2543.6 & 2595.048 & 11228.3 & 11268.79 & 7611.2 & 7688.619 \\
\hline $\begin{array}{l}\text { Cumulative } \\
\text { CRP } \\
\text { acres } \\
(2004)\end{array}$ & 5528 & 5511.076 & 3479.5 & 3503.208 & 2832 & 2837.895 & 10738.9 & 10729.59 & 8184.4 & 8166.032 \\
\hline $\begin{array}{l}\text { Cumulative } \\
\text { CRP } \\
\text { acres } \\
(2005)\end{array}$ & 5536.9 & 5526.292 & 3831 & 3782.267 & 3337.6 & 3320.542 & 10791.6 & 10808.65 & 8618.9 & 8678.791 \\
\hline $\begin{array}{l}\text { Cumulative } \\
\text { CRP } \\
\text { acres } \\
(2006)\end{array}$ & 5678.8 & 5758.297 & 3935.7 & 3966.847 & 3879.9 & 3638.254 & 11388 & 11380.06 & 9088 & 9040.823 \\
\hline $\begin{array}{l}\text { County } \\
\text { sizes }\end{array}$ & 383.91 & 483.2132 & 559.62 & 415.895 & 504.61 & 398.8533 & 758 & 534.68 & 646 & 421.655 \\
\hline $\begin{array}{l}\text { CRP } \\
\text { rental } \\
\text { rates }\end{array}$ & 483878 & 493100.5 & 341116.5 & 331601.5 & 272447.8 & 245475.2 & 1175159 & 859330.7 & 922286.1 & 8096965.6 \\
\hline
\end{tabular}


Figure 4.7: Jay, Indiana

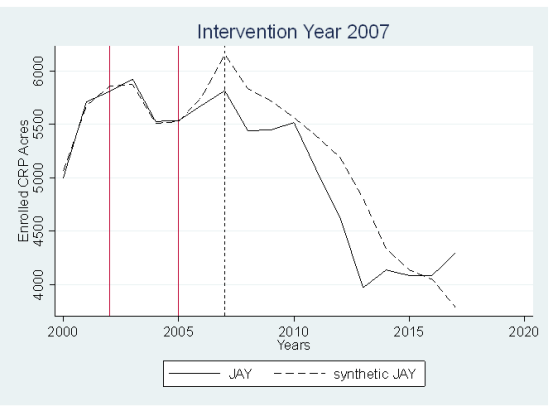

(a) Intervention Year

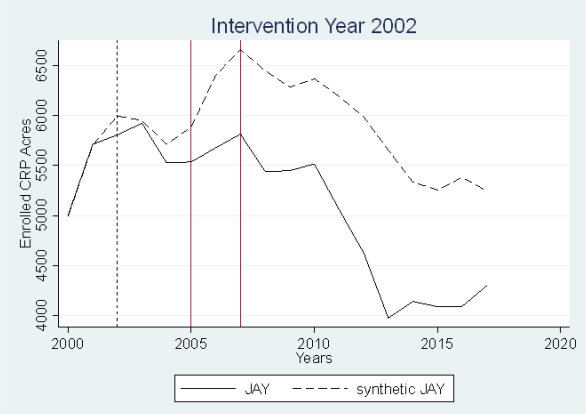

(b) In Time Placebo

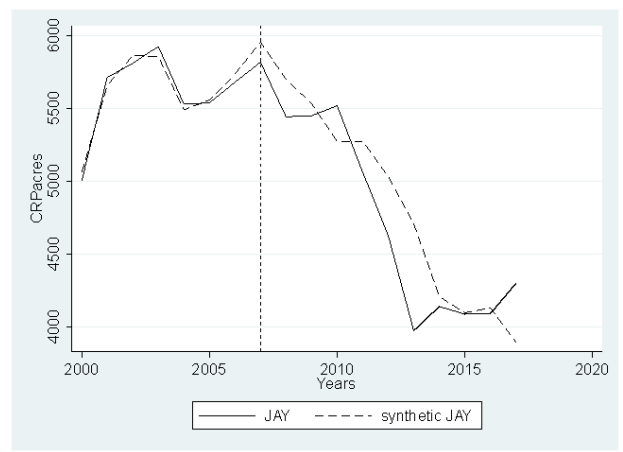

(c) Leave One Out 
Figure 4.8: Jasper, Indiana (CRP Acres)

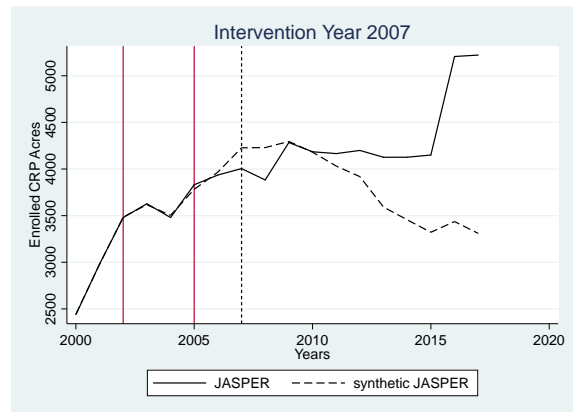

(a) Intervention Year

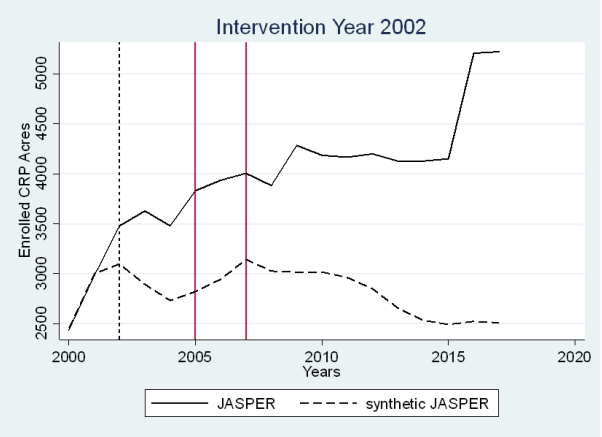

(b) In Time Placebo

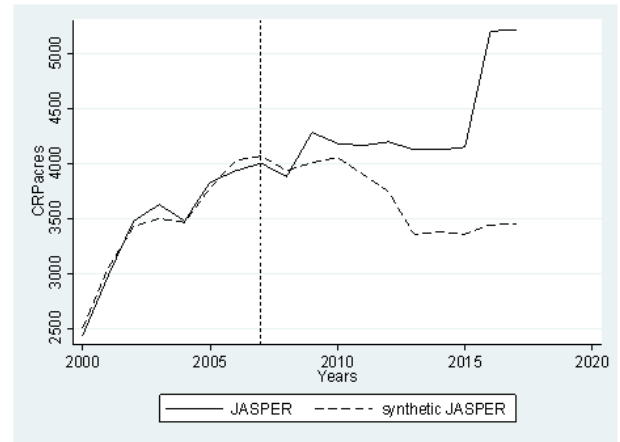

(c) Leave One Out 
Figure 4.9: Jasper, Indiana (Corn Acreage)

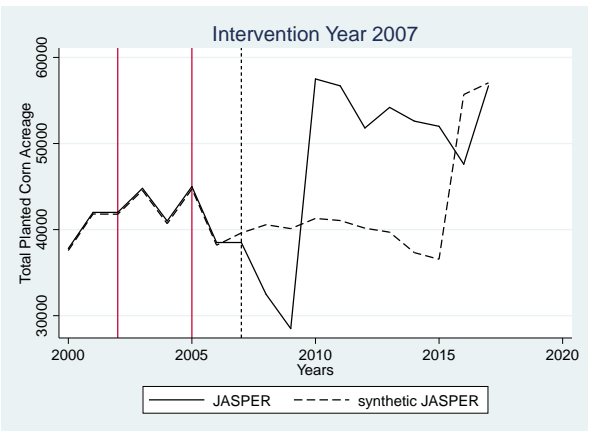

(a) Intervention Year

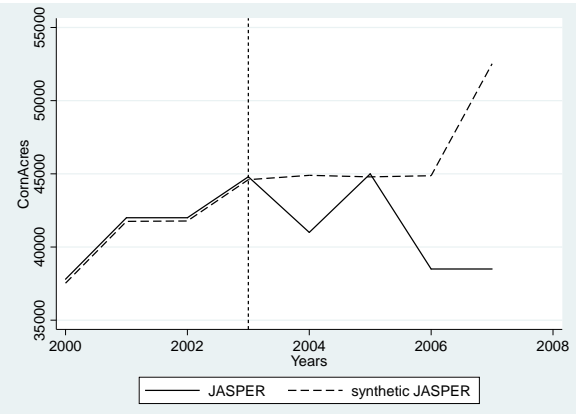

(b) In Time Placebo

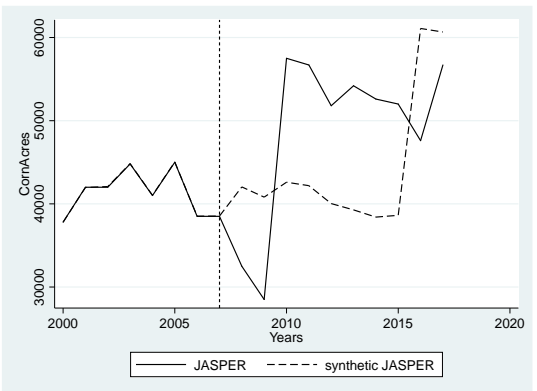

(c) Leave One Out 
Figure 4.10: Montgomery, Indiana

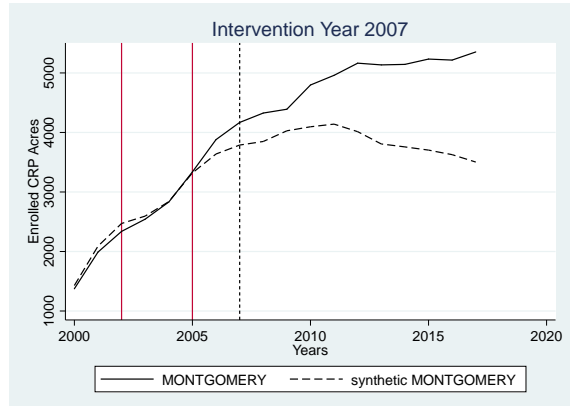

(a) Intervention Year

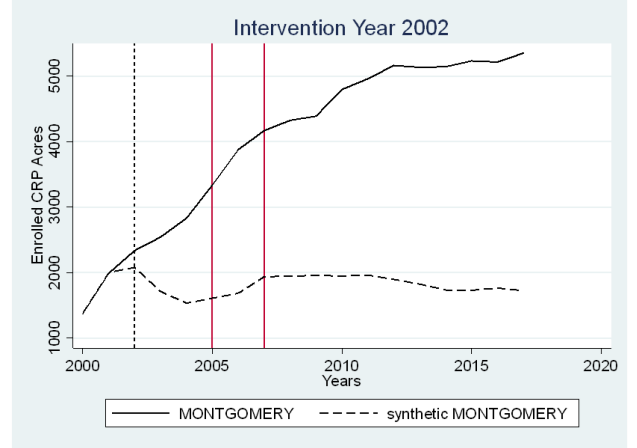

(b) In Time Placebo

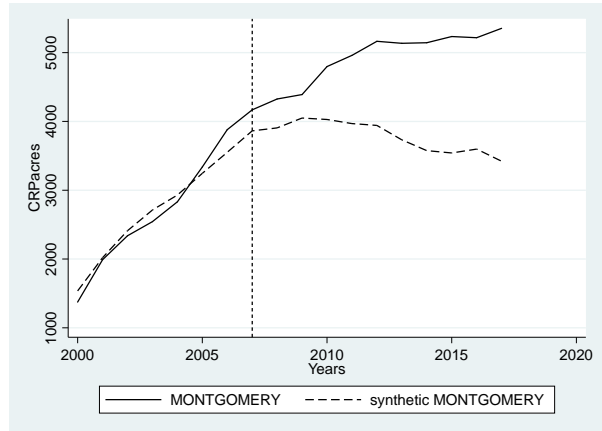

(c) Leave One Out 
Figure 4.11: Shelby, Indiana

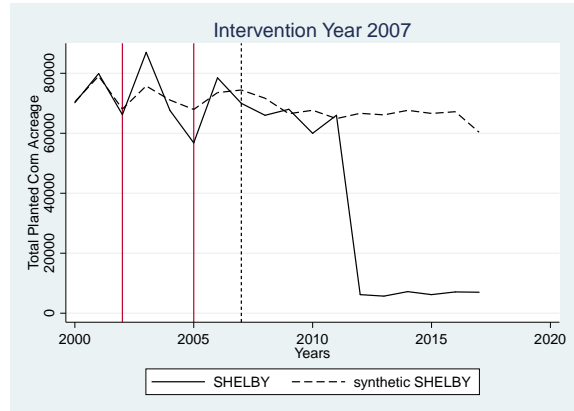

(a) Intervention Year

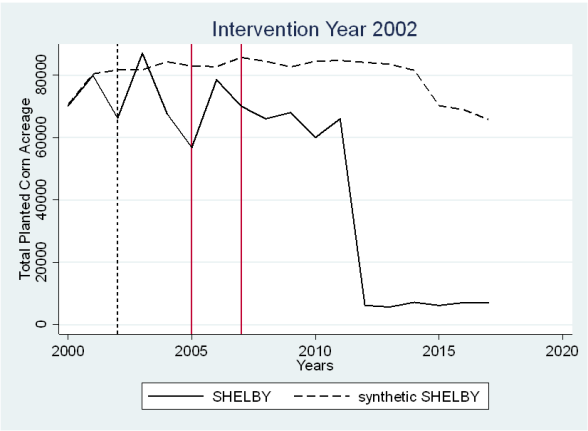

(b) In Time Placebo

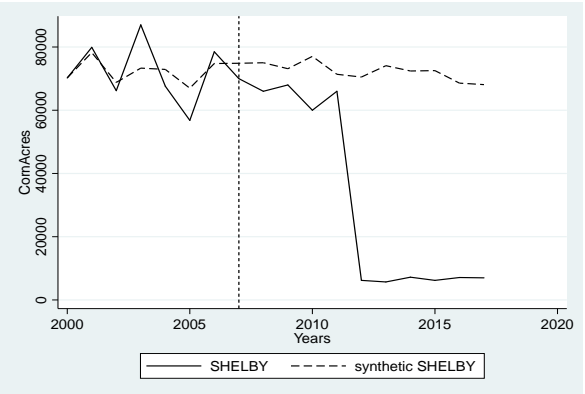

(c) Leave One Out 
Figure 4.12: Ogle, Illinois

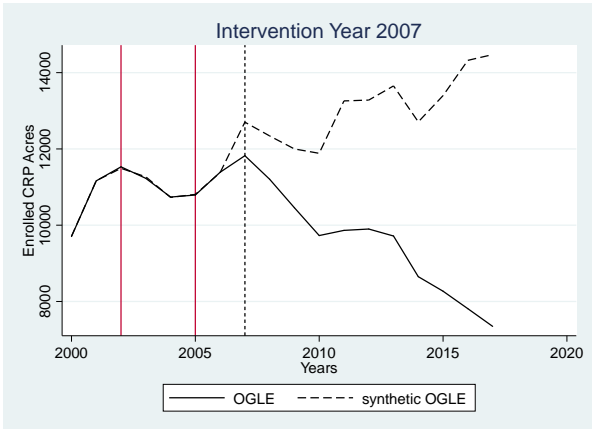

(a) Intervention Year

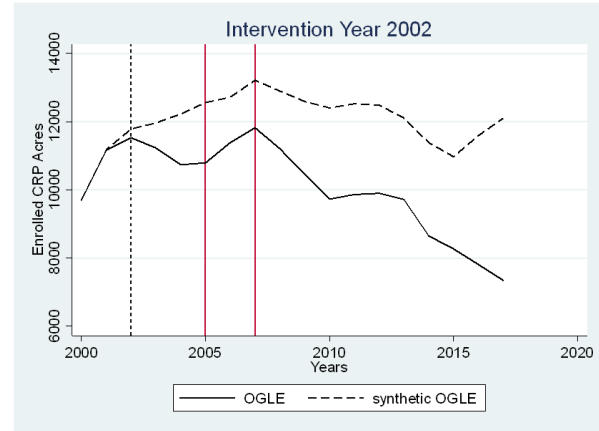

(b) In Time Placebo

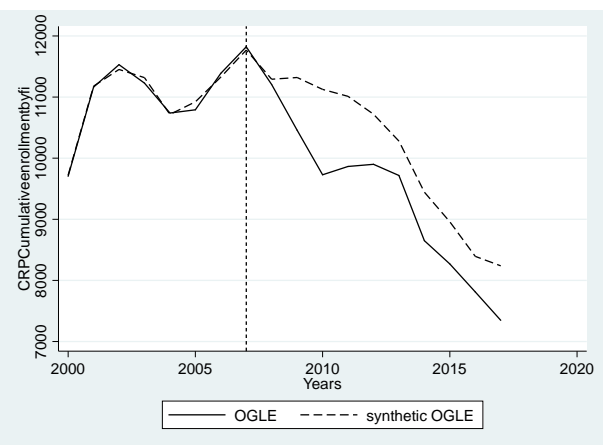

(c) Leave One Out 
Figure 4.13: Tazewell, Illinois

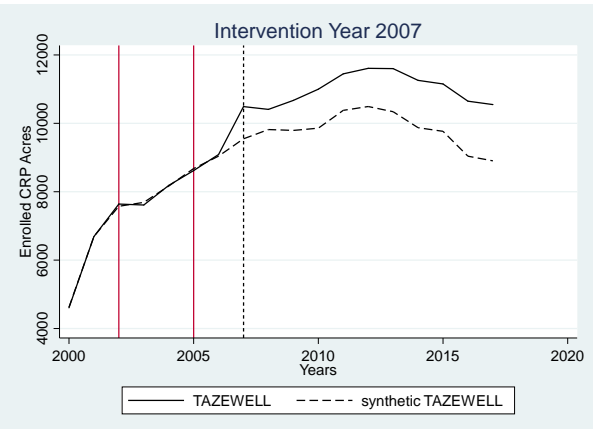

(a) Intervention Year

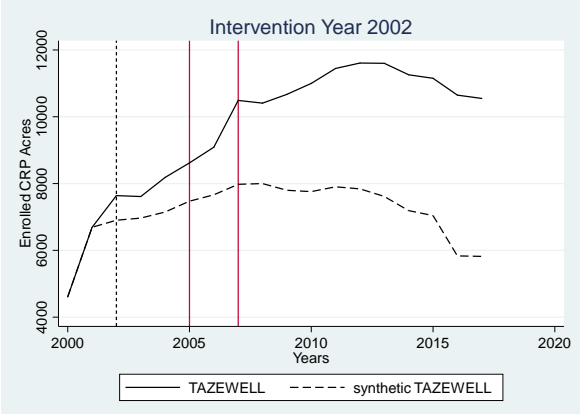

(b) In Time Placebo

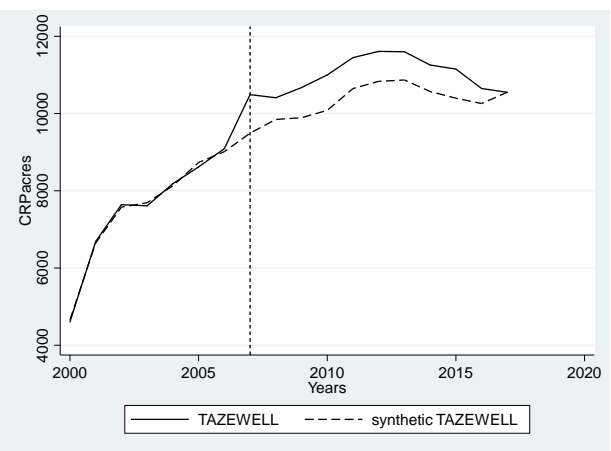

(c) Leave One Out 
Figure 4.14: Macon, Illinois

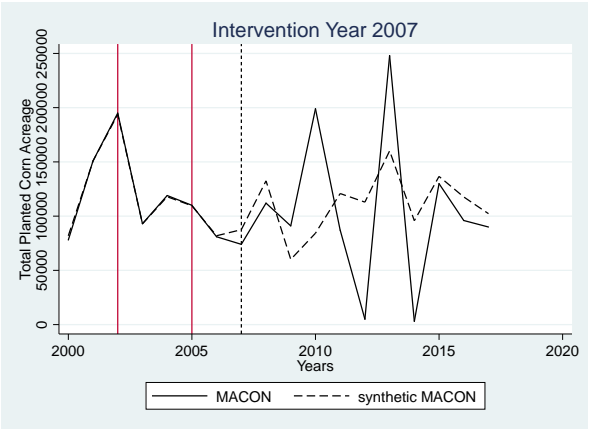

(a) Intervention Year

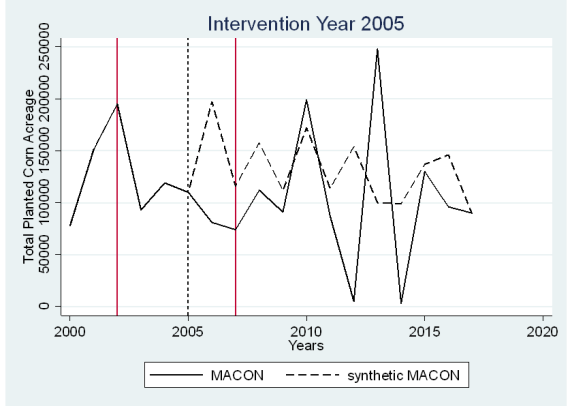

(b) In Time Placebo

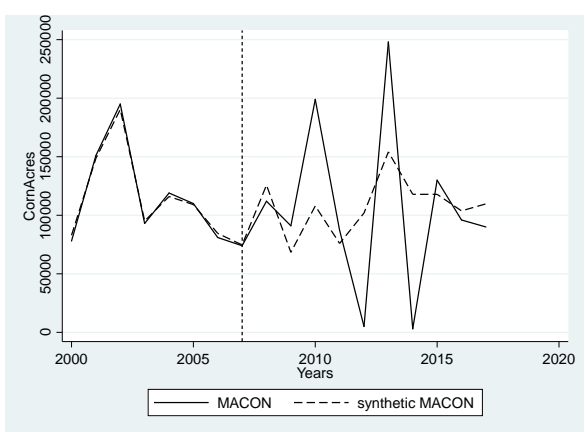

(c) Leave One Out 
Figure 4.15: Crawford, Illinois

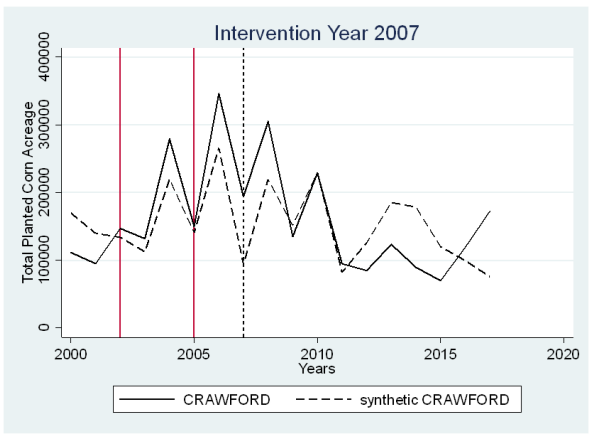

(a) Intervention Year

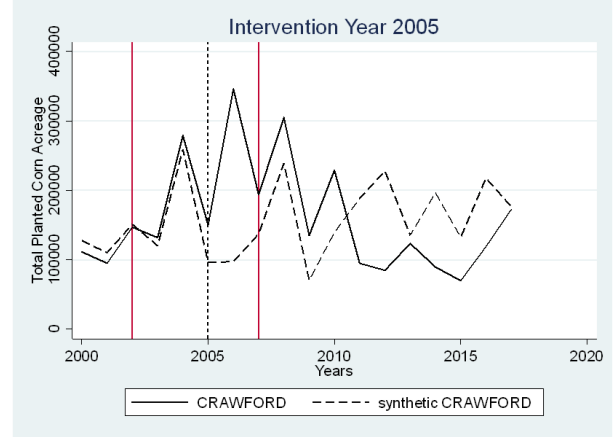

(b) In Time Placebo

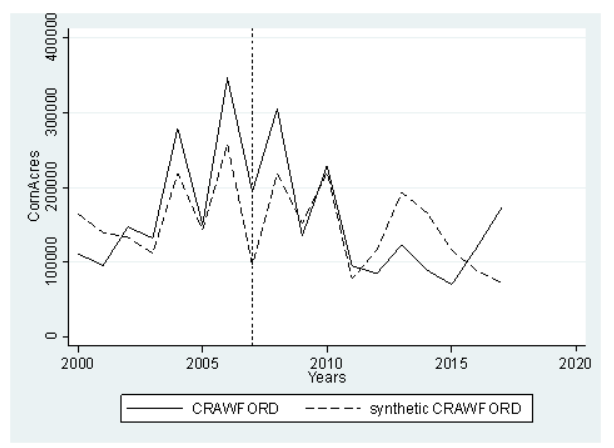

(c) Leave One Out 


\section{Chapter 5}

\section{Concluding Remarks}

In this dissertation, many of policy related issues are shown to need an integrated spatial analysis approach. For example, the first essay reveals that explicitly accounting for spatial drivers of food deserts is essential in finding places that have higher rates of chronic diseases due to a lack of food providers. Secondly, essay two shows how a switch in the way we account for spatial interlinkages among African countries can inform us about the underlying motives of traditional and non-tradition Foreign Direct Investments (FDI) in Africa. A historical colonial ties weight matrix is used to help understand how French, Dutch, American and Chinese FDIs might be operating on the African continent. Finally, the third essay shows how important it is to examine both the global and local effects of a policy intervention. When using a larger geographical unit (state level) in a Difference-in-Difference (DID) method, both acres enrolled in the Conservation Reserve Program (CRP) and total acres of planted corn are shown to increase after passage of the 2007 Energy Independence and Security Act with its implementation of Renewable Fuel Standards (RFS) mandating ethanol in gasoline. However, when the impacts of this policy are evaluated at a smaller geographical unit (county level) using a Synthetic Control Method (SCM), the case by case analysis showed heterogeneous impacts from this RFS mandate. Impacts on CRP and total corn planted acres varied by county and by post-intervention year, 
zeroing out the global effects found at the state level.

Overall, this dissertation shows that spatial economics, a sub-discipline of regional economics, is more than just bringing location, transport, and land into economics as Proost and Thisse (2019) suggest. Two such examples are demonstrated in this research. First, FDI in Africa can be explained by historical colonial ties rather than by strictly geographical location of countries. Secondly, public policy is shown to have spatially differentiated effects on both human health and environmental outcomes. In addition, research outcomes can change depending upon the geographical unit being assessed. In the end, the results of this dissertation demonstrate the advantages of including spatially explicit variables into policy analyses. 
Chapter 6

Appendices 
Table 6.1: Post-treatment Effects for Statistically Insignificant Counties in Indiana (Enrolled CRP Acres)

\begin{tabular}{|c|c|c|c|c|c|c|}
\hline & $\begin{array}{l}\text { Cumulative } \\
\text { CRP en- } \\
\text { rollment: } \\
\text { Davies } \\
\text { (Acres) }\end{array}$ & $\begin{array}{l}\text { Cumulative } \\
\text { CRP en- } \\
\text { rollment: } \\
\text { Posey } \\
\text { (Acres) }\end{array}$ & $\begin{array}{l}\text { Cumulative } \\
\text { CRP en- } \\
\text { rollment: } \\
\text { Putnam } \\
\text { (Acres) }\end{array}$ & $\begin{array}{l}\text { Cumulative } \\
\text { CRP en- } \\
\text { rollment: } \\
\text { Randolph } \\
\text { (Acres) }\end{array}$ & $\begin{array}{l}\text { Cumulative } \\
\text { CRP en- } \\
\text { rollment: } \\
\text { Grant } \\
\text { (Acres) }\end{array}$ & $\begin{array}{l}\text { Cumulative } \\
\text { CRP en- } \\
\text { rollment: } \\
\text { Wabash } \\
\text { (Acres) }\end{array}$ \\
\hline 2007 & $\begin{array}{l}-75.280 \\
(0.4395)\end{array}$ & $\begin{array}{r}-61.2841 \\
(0.5164)\end{array}$ & $\begin{array}{c}9.7907 \\
(0.9120)\end{array}$ & $\begin{array}{r}-47.6257 \\
(0.5934)\end{array}$ & $\begin{array}{r}-25.6061 \\
(0.8021)\end{array}$ & $\begin{array}{c}182.6719 \\
(0.2307)\end{array}$ \\
\hline 2008 & $\begin{array}{c}-361.5357 \\
(0.1648)\end{array}$ & $\begin{array}{c}-200.0266 \\
(0.3296)\end{array}$ & $\begin{array}{c}309.7072 \\
(0.1868)\end{array}$ & $\begin{array}{c}-113.7506 \\
(0.5714)\end{array}$ & $\begin{array}{c}172.6742 \\
(0.3736)\end{array}$ & $\begin{array}{c}-195.9098 \\
(0.3516)\end{array}$ \\
\hline 2009 & $\begin{array}{c}-475.3236 \\
(0.1648)\end{array}$ & $\begin{array}{c}-204.7273 \\
(0.4395)\end{array}$ & $\begin{array}{c}710.4572 \\
(0.1098)\end{array}$ & $\begin{array}{c}-208.7137 \\
(0.4505)\end{array}$ & $\begin{array}{c}351.2323 \\
(0.2747)\end{array}$ & $\begin{array}{c}-391.5051 \\
(0.2307)\end{array}$ \\
\hline 2010 & $\begin{array}{c}-455.7536 \\
(0.1978)\end{array}$ & $\begin{array}{c}-275.5059 \\
(0.4065)\end{array}$ & $\begin{array}{c}600.9408 \\
(0.1318)\end{array}$ & $\begin{array}{c}-352.4062 \\
(0.2967)\end{array}$ & $\begin{array}{c}384.5163 \\
(0.2417)\end{array}$ & $\begin{array}{c}-384.6374 \\
(0.2417)\end{array}$ \\
\hline 2011 & $\begin{array}{c}-375.2757 \\
(0.2307) \\
\end{array}$ & $\begin{array}{r}-363.701 \\
(0.2527) \\
\end{array}$ & $\begin{array}{c}1028.375 \\
(0.4175) \\
\end{array}$ & $\begin{array}{c}-350.5461 \\
(0.2747) \\
\end{array}$ & $\begin{array}{r}141.9272 \\
(0.6263) \\
\end{array}$ & $\begin{array}{c}-586.9681 \\
(0.1538) \\
\end{array}$ \\
\hline 2012 & $\begin{array}{r}-437.793 \\
(0.2307)\end{array}$ & $\begin{array}{l}-366.23 \\
(0.2967)\end{array}$ & $\begin{array}{r}1147.76 \\
(0.4249)\end{array}$ & $\begin{array}{r}-346.154 \\
(0.3516)\end{array}$ & $\begin{array}{r}-18.7664 \\
(0.9670)\end{array}$ & $\begin{array}{c}-695.2781 \\
(0.1208)\end{array}$ \\
\hline 2013 & $\begin{array}{r}-273.532 \\
(0.4725)\end{array}$ & $\begin{array}{c}-423.9125 \\
(0.2857)\end{array}$ & $\begin{array}{c}1381.115 \\
(0.6534)\end{array}$ & $\begin{array}{c}-389.6358 \\
(0.3406)\end{array}$ & $\begin{array}{c}113.2595 \\
(0.7252)\end{array}$ & $\begin{array}{c}-225.0886 \\
(0.5494)\end{array}$ \\
\hline 2014 & $\begin{array}{l}-95.688 \\
(0.8571) \\
\end{array}$ & $\begin{array}{c}-200.873 \\
(0.5934) \\
\end{array}$ & $\begin{array}{c}1453.116 \\
(0.3736)\end{array}$ & $\begin{array}{c}-429.2373 \\
(0.3296)\end{array}$ & $\begin{array}{l}85.4709 \\
(0.8571)\end{array}$ & $\begin{array}{c}128.5001 \\
(0.7692)\end{array}$ \\
\hline 2015 & $\begin{array}{c}16.110 \\
(0.9670) \\
\end{array}$ & $\begin{array}{r}-99.5634 \\
(0.8351) \\
\end{array}$ & $\begin{array}{c}1508.143 \\
(0.3956) \\
\end{array}$ & $\begin{array}{c}-420.0771 \\
(0.3406) \\
\end{array}$ & $\begin{array}{c}116.0573 \\
(0.7582) \\
\end{array}$ & $\begin{array}{c}232.9049 \\
(0.5714) \\
\end{array}$ \\
\hline 2016 & $\begin{array}{c}3.7990 \\
(0.9999)\end{array}$ & $\begin{array}{c}-116.7705 \\
(0.78021)\end{array}$ & $\begin{array}{c}1753.513 \\
(0.3516)\end{array}$ & $\begin{array}{c}-432.9923 \\
(0.3626)\end{array}$ & $\begin{array}{c}8.2847 \\
(0.1678)\end{array}$ & $\begin{array}{l}249.882 \\
(0.5054)\end{array}$ \\
\hline 2017 & $\begin{array}{c}148.9042 \\
(0.7582)\end{array}$ & $\begin{array}{c}-12.4708 \\
(0.9890)\end{array}$ & $\begin{array}{c}1241.725 \\
(0.4285)\end{array}$ & $\begin{array}{c}-424.7252 \\
(0.3956)\end{array}$ & $\begin{array}{c}-119.4998 \\
(0.7692)\end{array}$ & $\begin{array}{c}771.8564 \\
(0.2087)\end{array}$ \\
\hline
\end{tabular}

Notes: Statistical significance: "*” 10 percent, "**” 5 percent, "***" 1 percent. p-value in parentheses. These p-values are obtained by using the two-sided test from (Galiani and Quistorff, 2017) 
Table 6.2: Post-treatment Effects for Statistically Insignificant Counties in Indiana (Total Planted Corn Acres)

\begin{tabular}{|c|c|c|c|c|c|c|}
\hline & $\begin{array}{l}\text { Total } \\
\text { Planted } \\
\text { Corn: } \\
\text { Davies } \\
\text { (Acres) }\end{array}$ & $\begin{array}{l}\text { Total } \\
\text { Planted } \\
\text { Corn: } \\
\text { Posey } \\
\text { (Acres) }\end{array}$ & $\begin{array}{l}\text { Total } \\
\text { Planted } \\
\text { Corn: } \\
\text { Putnam } \\
\text { (Acres) }\end{array}$ & $\begin{array}{l}\text { Total } \\
\text { Planted } \\
\text { Corn: } \\
\text { Randolph } \\
\text { (Acres) }\end{array}$ & $\begin{array}{l}\text { Total } \\
\text { Planted } \\
\text { Corn: } \\
\text { Grant } \\
\text { (Acres) }\end{array}$ & $\begin{array}{l}\text { Total } \\
\text { Planted } \\
\text { Corn } \\
\text { Wabash } \\
\text { (Acres) }\end{array}$ \\
\hline 2007 & $\begin{array}{c}401.2 \\
(0.8681) \\
\end{array}$ & $\begin{array}{c}3168 \\
(0.6153) \\
\end{array}$ & $\begin{array}{l}-6415.2 \\
(0.3186) \\
\end{array}$ & $\begin{array}{l}-5139.3 \\
(0.3956) \\
\end{array}$ & $\begin{array}{c}11528 \\
(0.1428) \\
\end{array}$ & $\begin{array}{l}-7053.5 \\
(0.2527) \\
\end{array}$ \\
\hline 2008 & $\begin{array}{c}77.0017 \\
(0.9120)\end{array}$ & $\begin{array}{l}-4809.7 \\
(0.4065)\end{array}$ & $\begin{array}{c}6967.7 \\
(0.7472)\end{array}$ & $\begin{array}{l}10301.8 \\
(0.2087)\end{array}$ & $\begin{array}{c}1079.1 \\
(0.8241)\end{array}$ & $\begin{array}{c}-4999.4 \\
(0.4285)\end{array}$ \\
\hline 2009 & $\begin{array}{c}-155.9 \\
(0.9120)\end{array}$ & $\begin{array}{l}-1545.3 \\
(0.7692)\end{array}$ & $\begin{array}{c}1993.2 \\
(0.5714)\end{array}$ & $\begin{array}{c}-689 \\
(0.8681)\end{array}$ & $\begin{array}{c}4028.2 \\
(0.5384)\end{array}$ & $\begin{array}{c}-10428.4 \\
(0.2967)\end{array}$ \\
\hline 2010 & $\begin{array}{c}264.3 \\
(0.9120)\end{array}$ & $\begin{array}{l}-5243.9 \\
(0.4835)\end{array}$ & $\begin{array}{c}4299 \\
(0.4505)\end{array}$ & $\begin{array}{l}13019.8 \\
(0.2527)\end{array}$ & $\begin{array}{l}-3655.1 \\
(0.6043)\end{array}$ & $\begin{array}{c}-9743 \\
(0.3076)\end{array}$ \\
\hline 2011 & $\begin{array}{c}-234 \\
(0.8901) \\
\end{array}$ & $\begin{array}{c}-0.8 \\
(0.9340) \\
\end{array}$ & $\begin{array}{l}-5326.7 \\
(0.7582) \\
\end{array}$ & $\begin{array}{c}8442.7 \\
(0.3626) \\
\end{array}$ & $\begin{array}{l}-1227.4 \\
(0.8131) \\
\end{array}$ & $\begin{array}{r}-11703.5 \\
(0.2857) \\
\end{array}$ \\
\hline 2012 & $\begin{array}{c}-619.7 \\
(0.8681)\end{array}$ & $\begin{array}{c}316 \\
(0.9230)\end{array}$ & $\begin{array}{c}1821.8 \\
(0.4285)\end{array}$ & $\begin{array}{c}5111 \\
(0.6043)\end{array}$ & $\begin{array}{c}2592.2 \\
(0.7252)\end{array}$ & $\begin{array}{c}-7338 \\
(0.1208)\end{array}$ \\
\hline 2013 & $\begin{array}{c}102.6 \\
(0.9230)\end{array}$ & $\begin{array}{c}1560.2 \\
(0.7912)\end{array}$ & $\begin{array}{c}-9489.7 \\
(0.6263)\end{array}$ & $\begin{array}{c}5.9 \\
(0.9340)\end{array}$ & $\begin{array}{c}1277.7 \\
(0.8021)\end{array}$ & $\begin{array}{c}-14254 \\
(0.3076)\end{array}$ \\
\hline 2014 & $\begin{array}{c}227.1 \\
(0.9010)\end{array}$ & $\begin{array}{c}1511.1 \\
(0.8241)\end{array}$ & $\begin{array}{c}-5046.9 \\
(0.8241)\end{array}$ & $\begin{array}{c}4582.1 \\
(0.6373)\end{array}$ & $\begin{array}{c}1792.8 \\
(0.7912)\end{array}$ & $\begin{array}{l}-7116.5 \\
(0.5164)\end{array}$ \\
\hline 2015 & $\begin{array}{c}170.6 \\
(0.9230) \\
\end{array}$ & $\begin{array}{c}6335.3 \\
(0.6373) \\
\end{array}$ & $\begin{array}{c}-1392.2 \\
(0.3956)\end{array}$ & $\begin{array}{c}6537.1 \\
(0.6263) \\
\end{array}$ & $\begin{array}{c}3137.4 \\
(0.7582) \\
\end{array}$ & $\begin{array}{l}22951.5 \\
(0.5714) \\
\end{array}$ \\
\hline 2016 & $\begin{array}{c}-64.5 \\
(0.9340)\end{array}$ & $\begin{array}{c}-55764 \\
(0.1208)\end{array}$ & $\begin{array}{c}38361.9 \\
(0.1758)\end{array}$ & $\begin{array}{r}-18416.5 \\
(0.3186)\end{array}$ & $\begin{array}{c}10678.7 \\
(0.5534)\end{array}$ & $\begin{array}{c}23632.6 \\
(0.2747)\end{array}$ \\
\hline 2017 & $\begin{array}{l}-3285.5 \\
(0.8351)\end{array}$ & $\begin{array}{r}-41277.5 \\
(0.2307)\end{array}$ & $\begin{array}{c}37788.4 \\
(0.2637)\end{array}$ & $\begin{array}{c}-20798.1 \\
(0.4395)\end{array}$ & $\begin{array}{l}13232.7 \\
(0.6263)\end{array}$ & $\begin{array}{c}20301.2 \\
(0.0 .4615\end{array}$ \\
\hline
\end{tabular}

Notes: Statistical significance: "*" 10 percent, "**" 5 percent, "***” 1 percent. p-value in parentheses. These p-values are obtained by using the two-sided test from (Galiani and Quistorff, 2017) 
Table 6.3: Post-treatment Effects for Statistically Insignificant Counties in Illinois (Enrolled CRP Acres)

\begin{tabular}{|c|c|c|c|c|c|c|}
\hline & $\begin{array}{l}\text { Cumulative } \\
\text { CRP en- } \\
\text { rollment: } \\
\text { Stephenson } \\
\text { (Acres) }\end{array}$ & $\begin{array}{l}\text { Cumulative } \\
\text { CRP en- } \\
\text { rollment: } \\
\text { Peoria } \\
\text { (Acres) }\end{array}$ & $\begin{array}{l}\text { Cumulative } \\
\text { CRP en- } \\
\text { rollment: } \\
\text { Putnam } \\
\text { (Acres) }\end{array}$ & $\begin{array}{l}\text { Cumulative } \\
\text { CRP en- } \\
\text { rollment: } \\
\text { Madison } \\
\text { (Acres) }\end{array}$ & $\begin{array}{l}\text { Cumulative } \\
\text { CRP en- } \\
\text { rollment: } \\
\text { Henry } \\
\text { (Acres) }\end{array}$ & $\begin{array}{l}\text { Cumulative } \\
\text { CRP en- } \\
\text { rollment: } \\
\text { Ford } \\
\text { (Acres) }\end{array}$ \\
\hline 2007 & $\begin{array}{c}-158.8249 \\
(0.6831)\end{array}$ & $\begin{array}{c}60.6642 \\
(0.8910)\end{array}$ & $\begin{array}{c}-31.827 \\
(0.9207)\end{array}$ & $\begin{array}{r}323.8217 \\
(0.3564)\end{array}$ & $\begin{array}{c}571.0092 \\
(0.1782)\end{array}$ & $\begin{array}{c}-267.6165 \\
(0.4356)\end{array}$ \\
\hline 2008 & $\begin{array}{c}-160.9669 \\
(0.7524)\end{array}$ & $\begin{array}{c}436.7316 \\
(0.4752)\end{array}$ & $\begin{array}{r}-114.683 \\
(0.8217)\end{array}$ & $\begin{array}{c}338.1505 \\
(0.5346)\end{array}$ & $\begin{array}{c}313.7728 \\
(0.5445)\end{array}$ & $\begin{array}{c}-185.6456 \\
(0.7425)\end{array}$ \\
\hline 2009 & $\begin{array}{r}-111.421 \\
(0.8712)\end{array}$ & $\begin{array}{c}468.8608 \\
(0.4851)\end{array}$ & $\begin{array}{c}-297.6203 \\
(0.6633)\end{array}$ & $\begin{array}{c}208.476 \\
(0.7227)\end{array}$ & $\begin{array}{r}445.7497 \\
(0.5346)\end{array}$ & $\begin{array}{c}-439.2638 \\
(0.5643)\end{array}$ \\
\hline 2010 & $\begin{array}{c}-221.7787 \\
(0.7821)\end{array}$ & $\begin{array}{c}633.4673 \\
(0.4653)\end{array}$ & $\begin{array}{c}-344.1259 \\
(0.6831)\end{array}$ & $\begin{array}{c}27.9213 \\
(0.9603)\end{array}$ & $\begin{array}{c}-127.4123 \\
(0.8514)\end{array}$ & $\begin{array}{c}-458.4757 \\
(0.5445)\end{array}$ \\
\hline 2011 & $\begin{array}{c}551.9713 \\
(0.5940)\end{array}$ & $\begin{array}{c}963.0465 \\
(0.4158)\end{array}$ & $\begin{array}{c}-327.4761 \\
(0.7029)\end{array}$ & $\begin{array}{c}346.0351 \\
(0.7029)\end{array}$ & $\begin{array}{c}822.3756 \\
(0.4554)\end{array}$ & $\begin{array}{c}-606.4553 \\
(0.5643)\end{array}$ \\
\hline 2012 & $\begin{array}{r}2068.521 \\
(0.1683)\end{array}$ & $\begin{array}{c}1160.816 \\
(0.3960)\end{array}$ & $\begin{array}{c}-337.1383 \\
(0.7524)\end{array}$ & $\begin{array}{c}636.185 \\
(0.6336)\end{array}$ & $\begin{array}{c}1428.22 \\
(0.3168)\end{array}$ & $\begin{array}{c}-733.9815 \\
(0.5841)\end{array}$ \\
\hline 2013 & $\begin{array}{c}2281.863 \\
(0.1980)\end{array}$ & $\begin{array}{c}1378.692 \\
(0.3663)\end{array}$ & $\begin{array}{c}-303.4894 \\
(0.7920)\end{array}$ & $\begin{array}{c}792.6234 \\
(0.6039)\end{array}$ & $\begin{array}{c}1917.483 \\
(0.2227)\end{array}$ & $\begin{array}{c}-666.9167 \\
(0.6435)\end{array}$ \\
\hline 2014 & $\begin{array}{c}2087 \\
(0.2277)\end{array}$ & $\begin{array}{c}1507.036 \\
(0.3861)\end{array}$ & $\begin{array}{c}-210.1103 \\
(0.8613)\end{array}$ & $\begin{array}{c}767.2249 \\
(0.5940)\end{array}$ & $\begin{array}{l}1049.85 \\
(0.5346)\end{array}$ & $\begin{array}{l}-5.5550 \\
(0.9603)\end{array}$ \\
\hline 2015 & $\begin{array}{c}2242.902 \\
(0.2475)\end{array}$ & $\begin{array}{c}1222.095 \\
(0.4950)\end{array}$ & $\begin{array}{c}-219.4173 \\
(0.8613)\end{array}$ & $\begin{array}{c}747.0396 \\
(0.6435)\end{array}$ & $\begin{array}{c}1091.142 \\
(0.5346)\end{array}$ & $\begin{array}{c}413.181 \\
(0.7524)\end{array}$ \\
\hline 2016 & $\begin{array}{c}2735.569 \\
(0.2673)\end{array}$ & $\begin{array}{c}1432.496 \\
(0.5148)\end{array}$ & $\begin{array}{c}-2109.508 \\
(0.3564)\end{array}$ & $\begin{array}{c}850.8769 \\
(0.6633)\end{array}$ & $\begin{array}{c}2248.695 \\
(0.3366)\end{array}$ & $\begin{array}{c}1319.645 \\
(0.5445)\end{array}$ \\
\hline 2017 & $\begin{array}{c}2970.355 \\
(0.3267)\end{array}$ & $\begin{array}{c}1567.064 \\
(0.5346)\end{array}$ & $\begin{array}{c}-2238.856 \\
(0.3960)\end{array}$ & $\begin{array}{c}758.6163 \\
(0.7326)\end{array}$ & $\begin{array}{c}3512 \\
(0.2574)\end{array}$ & $\begin{array}{c}2822.012 \\
(0.3366)\end{array}$ \\
\hline
\end{tabular}

Notes: Statistical significance: "*” 10 percent, "**" 5 percent, "***" 1 percent. p-value in parentheses. These p-values are obtained by using the two-sided test from (Galiani and Quistorff, 2017) 
Table 6.4: Post-treatment Effects for Statistically Insignificant Counties in Illinois (Total Planted Corn Acres)

\begin{tabular}{|c|c|c|c|c|c|c|}
\hline & $\begin{array}{l}\text { Total } \\
\text { Planted } \\
\text { Corn: } \\
\text { Stephenson } \\
\text { (Acres) }\end{array}$ & $\begin{array}{l}\text { Total } \\
\text { Planted } \\
\text { Corn: } \\
\text { Peoria } \\
\text { (Acres) }\end{array}$ & $\begin{array}{l}\text { Total } \\
\text { Planted } \\
\text { Corn: } \\
\text { Putnam } \\
\text { (Acres) }\end{array}$ & $\begin{array}{l}\text { Total } \\
\text { Planted } \\
\text { Corn: } \\
\text { Madison } \\
\text { (Acres) }\end{array}$ & $\begin{array}{l}\text { Total } \\
\text { Planted } \\
\text { Corn: } \\
\text { Henry } \\
\text { (Acres) }\end{array}$ & $\begin{array}{l}\text { Total } \\
\text { Planted } \\
\text { Corn: } \\
\text { Ford } \\
\text { (Acres) }\end{array}$ \\
\hline 2007 & $\begin{array}{r}-12211.1 \\
(0.5742)\end{array}$ & $\begin{array}{l}-4203.4 \\
(0.7722)\end{array}$ & $\begin{array}{c}-5027 \\
(0.7524)\end{array}$ & $\begin{array}{c}3223 \\
(0.7821)\end{array}$ & $\begin{array}{c}-4896.5 \\
(0.7623)\end{array}$ & $\begin{array}{c}-2945 \\
(0.8019)\end{array}$ \\
\hline 2008 & $\begin{array}{r}-17250.6 \\
(0.4156)\end{array}$ & $\begin{array}{c}-22932.5 \\
(0.3564)\end{array}$ & $\begin{array}{c}-2995 \\
(0.9207)\end{array}$ & $\begin{array}{c}6586.5 \\
(0.6930)\end{array}$ & $\begin{array}{c}-8057 \\
(0.6336)\end{array}$ & $\begin{array}{c}-5551 \\
(0.7623)\end{array}$ \\
\hline 2009 & $\begin{array}{c}-8897.1 \\
(0.6039)\end{array}$ & $\begin{array}{l}13688.2 \\
(0.4356)\end{array}$ & $\begin{array}{c}3787 \\
(0.8019)\end{array}$ & $\begin{array}{c}4770.8 \\
(0.7425)\end{array}$ & $\begin{array}{c}-1271 \\
(0.9108)\end{array}$ & $\begin{array}{c}2962 \\
(0.8415)\end{array}$ \\
\hline 2010 & $\begin{array}{c}29999.8 \\
(0.2970)\end{array}$ & $\begin{array}{c}67087.6 \\
(0.1386)\end{array}$ & $\begin{array}{c}2772 \\
(0.8811)\end{array}$ & $\begin{array}{r}-12991.5 \\
(0.4752)\end{array}$ & $\begin{array}{r}-23080.1 \\
(0.3465)\end{array}$ & $\begin{array}{c}4299 \\
(0.7920)\end{array}$ \\
\hline 2011 & $\begin{array}{r}-26536.2 \\
(0.3564) \\
\end{array}$ & $\begin{array}{r}-19490.9 \\
(0.4455) \\
\end{array}$ & $\begin{array}{c}5678 \\
(0.7623) \\
\end{array}$ & $\begin{array}{c}4914.5 \\
(0.8019) \\
\end{array}$ & $\begin{array}{r}10832.4 \\
(0.6039) \\
\end{array}$ & $\begin{array}{c}5187 \\
(0.7722) \\
\end{array}$ \\
\hline 2012 & $\begin{array}{r}-46034.5 \\
(0.2475)\end{array}$ & $\begin{array}{r}-77885.7 \\
(0.1980)\end{array}$ & $\begin{array}{c}9309 \\
(0.6237)\end{array}$ & $\begin{array}{l}-7736.6 \\
(0.6930)\end{array}$ & $\begin{array}{c}-23048.3 \\
(0.4754)\end{array}$ & $\begin{array}{c}32033 \\
(0.3762)\end{array}$ \\
\hline 2013 & $\begin{array}{c}25462 \\
(0.4059)\end{array}$ & $\begin{array}{l}43504.3 \\
(0.2376\end{array}$ & $\begin{array}{c}1493 \\
(0.9207)\end{array}$ & $\begin{array}{c}-8008 \\
(0.6633)\end{array}$ & $\begin{array}{c}3430.2 \\
(0.8712)\end{array}$ & $\begin{array}{c}16958 \\
(0.495)\end{array}$ \\
\hline 2014 & $\begin{array}{r}-41282.8 \\
(0.3069)\end{array}$ & $\begin{array}{r}-60141.8 \\
(0.1485)\end{array}$ & $\begin{array}{c}6493 \\
(0.8118)\end{array}$ & $\begin{array}{c}-9638 \\
(0.6831)\end{array}$ & $\begin{array}{c}-2424 \\
(0.4752)\end{array}$ & $\begin{array}{c}14950 \\
(0.6138)\end{array}$ \\
\hline 2015 & $\begin{array}{c}-15215 \\
(0.4354) \\
\end{array}$ & $\begin{array}{r}-16669.8 \\
(0.4257) \\
\end{array}$ & $\begin{array}{c}26687 \\
(0.3168) \\
\end{array}$ & $\begin{array}{c}-448.2 \\
(0.9405) \\
\end{array}$ & $\begin{array}{c}-533.5 \\
(0.9306) \\
\end{array}$ & $\begin{array}{c}1344 \\
(0.9009)\end{array}$ \\
\hline 2016 & $\begin{array}{r}-11049.5 \\
(0.6435)\end{array}$ & $\begin{array}{c}-9890.1 \\
(0.6732)\end{array}$ & $\begin{array}{c}6501.6 \\
(0.8019)\end{array}$ & $\begin{array}{c}-7388.2 \\
(0.7623)\end{array}$ & $\begin{array}{c}-14791 \\
(0.5346)\end{array}$ & $\begin{array}{c}19321 \\
(0.4455)\end{array}$ \\
\hline 2017 & $\begin{array}{r}-16887.1 \\
(0.4950)\end{array}$ & $\begin{array}{c}-8935 \\
(0.7029)\end{array}$ & $\begin{array}{c}2449 \\
(0.8712)\end{array}$ & $\begin{array}{c}2767.2 \\
(0.8613)\end{array}$ & $\begin{array}{c}26346.3 \\
(0.3663)\end{array}$ & $\begin{array}{c}13284.4 \\
(0.5742)\end{array}$ \\
\hline
\end{tabular}

Notes: Statistical significance: "*" 10 percent, "**" 5 percent, "***” 1 percent. p-value in parentheses. These p-values are obtained by using the two-sided test from (Galiani and Quistorff, 2017) 


\section{Bibliography}

Abadie, A. (2021). Using synthetic controls: Feasibility, data requirements, and methodological aspects. Journal of Economic Literature, 59(2):391-425.

Abadie, A., Diamond, A., and Hainmueller, J. (2010). Synthetic control methods for comparative case studies: Estimating the effect of california's tobacco control program. Journal of the American statistical Association, 105(490):493-505.

Abadie, A., Diamond, A., and Hainmueller, J. (2015). Comparative politics and the synthetic control method. American Journal of Political Science, 59(2):495-510.

Abadie, A. and Gardeazabal, J. (2003). The economic costs of conflict: A case study of the basque country. American economic review, 93(1):113-132.

Acemoglu, D., Johnson, S., and Robinson, J. A. (2001). The colonial origins of comparative development: An empirical investigation. American economic review, 91(5):1369-1401.

Allcott, H., Diamond, R., Dubé, J.-P., Handbury, J., Rahkovsky, I., and Schnell, M. (2019). Food deserts and the causes of nutritional inequality. The Quarterly Journal of Economics, 134(4):1793-1844.

Alpert, A. (2016). The anticipatory effects of medicare part d on drug utilization. Journal of health economics, 49:28-45.

Alviola, P. A., Nayga, R. M., Thomsen, M. R., and Wang, Z. (2013). Determinants of food deserts. American Journal of Agricultural Economics, 95(5):1259-1265.

Ando, M. (2015). Dreams of urbanization: Quantitative case studies on the local impacts of nuclear power facilities using the synthetic control method. Journal of Urban Economics, 85:68-85.

Andress, L. (2017). Using a social ecological model to explore upstream and downstream solutions to rural food access for the elderly. Cogent Medicine, 4(1):1393849. 
Andress, L. and Fitch, C. (2016). Juggling the five dimensions of food access: Perceptions of rural low income residents. Appetite, 105:151-155.

Andress, L. and Hallie, S. S. (2017). Co-constructing food access issues: Older adults in a rural food environment in west virginia develop a photonarrative. Cogent Medicine, 4(1):1309804.

Annen, K. and Knack, S. (2019). Better Policies from Policy-Selective Aid? The World Bank.

Arora, G., Wolter, P. T., Feng, H., and Hennessy, D. A. (2016). Role of ethanol plants in dakotas land use change: Incorporating flexible trends in the differencein-difference framework with remotely-sensed data. Working Paper.

Asiedu, E. (2006). Foreign Direct Investment in Africa: The Role of Natural Resources, Market Size, Government Policy, Institutions and Political Instability. The World Economy, 29(1):63-77.

Bailey, J. M. (2010). Rural grocery stores: Importance and challenges. Center for Rural Affairs Rural Research and Analysis Program, 10.

Baltagi, B. H., Egger, P., and Pfaffermayr, M. (2007). Estimating Models of Complex FDI: Are there Third-country Effects? Journal of Econometrics, 140(1):260-281. Analysis of spatially dependent data.

Blanco, L. R. (2012). The Spatial Interdependence of FDI in Latin America. World Development, 40(7):1337-1351.

Blonigen, B. A., Davies, R. B., Waddell, G. R., and Naughton, H. T. (2007). FDI in Space: Spatial Autoregressive Relationships in Foreign Direct Investment. European Economic Review, 51(5):1303-1325.

Bracmort, K. (2015). The renewable fuel standard (rfs): Cellulosic biofuels. Congressional Research Service, Washington, DC, accessed Apr, 3:2018. 
Brautigama, D. and Ackerb, K. (2021). Chinese lending to africa in the pandemic era. Shaping Africa's Post-Covid Recovery.

Brown, J. C., Hanley, E., Bergtold, J., Caldas, M., Barve, V., Peterson, D., Callihan, R., Gibson, J., Gray, B., Hendricks, N., et al. (2014). Ethanol plant location and intensification vs. extensification of corn cropping in kansas. Applied Geography, $53: 141-148$.

Caillavet, F., Kyureghian, G., Nayga Jr, R. M., Ferrant, C., and Chauvin, P. (2015). Does healthy food access matter in a french urban setting? American Journal of Agricultural Economics, 97(5):1400-1416.

Carr, D. L., Markusen, J. R., and Maskus, K. E. (2001). Estimating the knowledgecapital model of the multinational enterprise. American Economic Review, 91(3):693-708.

Carter, C. A., Rausser, G. C., and Smith, A. (2017). Commodity storage and the market effects of biofuel policies. American Journal of Agricultural Economics, 99(4):1027-1055.

Caspi, C. E., Sorensen, G., Subramanian, S., and Kawachi, I. (2012). The local food environment and diet: a systematic review. Health \& place, 18(5):1172-1187.

Chen, D., Jaenicke, E. C., and Volpe, R. J. (2016). Food environments and obesity: household diet expenditure versus food deserts. American journal of public health, 106(5):881-888.

Chen, L., Debnath, D., Zhong, J., Ferin, K., VanLoocke, A., and Khanna, M. (2021). The economic and environmental costs and benefits of the renewable fuel standard. Environmental Research Letters, 16(3):034021.

Chen, W., Dollar, D., and Tang, H. (2018). Why Is China Investing in Africa? Evidence from the Firm Level. World Bank Economic Review, 32(3):610-632. 
Chen, X. and Khanna, M. (2018). Effect of corn ethanol production on conservation reserve program acres in the us. Applied Energy, 225:124-134.

Christine, P. J., Auchincloss, A. H., Bertoni, A. G., Carnethon, M. R., Sánchez, B. N., Moore, K., Adar, S. D., Horwich, T. B., Watson, K. E., and Roux, A. V. D. (2015). Longitudinal associations between neighborhood physical and social environments and incident type 2 diabetes mellitus: the multi-ethnic study of atherosclerosis (mesa). JAMA internal medicine, 175(8):1311-1320.

Cooksey-Stowers, K., Schwartz, M. B., and Brownell, K. D. (2017). Food swamps predict obesity rates better than food deserts in the united states. International journal of environmental research and public health, 14(11):1366.

Creel, J. S., Sharkey, J. R., McIntosh, A., Anding, J., and Huber, J. C. (2008). Availability of healthier options in traditional and nontraditional rural fast-food outlets. BMC Public Health, 8(1):1-9.

Cummins, S., Flint, E., and Matthews, S. A. (2014). New neighborhood grocery store increased awareness of food access but did not alter dietary habits or obesity. Health affairs, 33(2):283-291.

Cummins, S. and Macintyre, S. (2002). "food deserts" - evidence and assumption in health policy making. Bmj, 325(7361):436-438.

Currie, J. and Walker, R. (2011). Traffic congestion and infant health: Evidence from e-zpass. American Economic Journal: Applied Economics, 3(1):65-90.

Deb, P. and Vargas, C. (2016). Who benefits from calorie labeling? an analysis of its effects on body mass. Technical report, National Bureau of Economic Research.

Deskins, J. et al. (2018). The economic impact of coal in west virginia.

Donou-Adonsou, F. and Lim, S. (2018). On the Importance of Chinese Investment in Africa. Review of Development Finance, 8(1):63-73. 
Doudchenko, N. and Imbens, G. W. (2016). Balancing, regression, difference-indifferences and synthetic control methods: A synthesis. Technical report, National Bureau of Economic Research.

Dreher, A., Fuchs, A., Hodler, R., Parks, B., Raschky, P., and Tierney, M. J. (2016). Aid on Demand: African Leaders and the Geography of China's Foreign Assistance. Centro Studi Luca d'Agliano Development Studies Working Paper, (400).

Dreher, A., Fuchs, A., Hodler, R., Parks, B. C., Raschky, P. A., and Tierney, M. J. (2019). African Leaders and the Geography of China's Foreign Assistance. Journal of Development Economics, 140:44-71.

Dreher, A., Fuchs, A., Hodler, R., Parks, B. C., Raschky, P. A., and Tierney, M. J. (2021a). Is favoritism a threat to chinese aid effectiveness? a subnational analysis of chinese development projects. World Development, 139:105291.

Dreher, A., Fuchs, A., Parks, B., Strange, A., and Tierney, M. J. (2021b). Aid, China, and Growth: Evidence from a New Global Development Finance Dataset. American Economic Journal: Economic Policy, 13(2):135-74.

Dreher, A., Fuchs, A., Parks, B., Strange, A. M., and Tierney, M. J. (2018). Apples and Dragon Fruits: The Determinants of Aid and Other Forms of State Financing from China to Africa. International Studies Quarterly, 62(1):182-194.

Egger, P. and Merlo, V. (2007). The impact of bilateral investment treaties on fdi dynamics. World Economy, 30(10):1536-1549.

Elhorst, J. P. (2014). Dynamic Spatial Panels: Models, Methods and Inferences. In Spatial econometrics, pages 95-119. Springer.

Englebert, P. (2000). Pre-colonial Institutions, Post-colonial States, and Economic Development in Tropical Africa. Political Research Quarterly, 53(1):7-36.

Eom, J., Hwang, J., Atkins, L., Chen, Y., and Zhou, S. (2017). The united states and china in africa: What does the data say? Policy Brief, 18. 
Foster, V. (2009). Building bridges: China's growing role as infrastructure financier for Sub-Saharan Africa, volume 5. World Bank Publications.

Galiani, S. and Quistorff, B. (2017). The synth_runner package: Utilities to automate synthetic control estimation using synth. The Stata Journal, 17(4):834-849.

Ganau, R. (2017). Institutions and Economic Growth in Africa: A Spatial Econometric Approach. Economia Politica, 34(3):425-444.

Gebreab, S. Y., Hickson, D. A., Sims, M., Wyatt, S. B., Davis, S. K., Correa, A., and Diez-Roux, A. V. (2017). Neighborhood social and physical environments and type 2 diabetes mellitus in african americans: The jackson heart study. Health $\&$ place, $43: 128-137$.

Grier, R. M. (1999). Colonial Legacies and Economic Growth. Public Choice, 98(3):317-335.

Grossman, D. S., Humphreys, B. R., and Ruseski, J. E. (2019). Out of the outhouse: The impact of place-based policies on dwelling characteristics in appalachia. Journal of Regional Science, 59(1):5-28.

Gulley, A. L., McCullough, E. A., and Shedd, K. B. (2019). China's domestic and foreign influence in the global cobalt supply chain. Resources Policy, 62:317-323.

Hager, E. R., Cockerham, A., O’Reilly, N., Harrington, D., Harding, J., Hurley, K. M., and Black, M. M. (2017). Food swamps and food deserts in baltimore city, md, usa: Associations with dietary behaviours among urban adolescent girls. Public health nutrition, 20(14):2598-2607.

Hainmueller, J. (2012). Entropy balancing for causal effects: A multivariate reweighting method to produce balanced samples in observational studies. Political analysis, 20(1):25-46.

Hasnat, B. (2013). The US and Chinese Foreign Direct Investment in Africa. ChinaUSA Business Review, 12(8). 
Hellerstein, D. M. (2017). The us conservation reserve program: The evolution of an enrollment mechanism. Land Use Policy, 63:601-610.

Hendricks, N. P. and Er, E. (2018). Changes in cropland area in the united states and the role of crp. Food Policy, 75:15-23.

Huang, K.-M. and Etienne, X. (2021). Impact of marcellus and utica shale exploitation on ohio, pennsylvania, and west virginia regional economies: A synthetic control analysis. Papers in Regional Science.

Ifft, J., Rajagopal, D., and Weldzuis, R. (2019). Ethanol plant location and land use: A case study of crp and the ethanol mandate. Applied Economic Perspectives and Policy, 41(1):37-55.

Jakus, P. M. and Akhundjanov, S. B. (2019). The antiquities act, national monuments, and the regional economy. Journal of Environmental Economics and Management, 95:102-117.

Jang, S. and Kim, J. (2018). Remedying food policy invisibility with spatial intersectionality: A case study in the detroit metropolitan area. Journal of Public Policy \& Marketing, 37(1):167-187.

Jilcott Pitts, S. B., McGuirt, J. T., Carr, L. J., Wu, Q., and Keyserling, T. C. (2012). Associations between body mass index, shopping behaviors, amenity density, and characteristics of the neighborhood food environment among female adult supplemental nutrition assistance program (snap) participants in eastern north carolina. Ecology of food and nutrition, 51(6):526-541.

Johnson, D. B., Quinn, E., Sitaker, M., Ammerman, A., Byker, C., Dean, W., Fleischhacker, S., Kolodinsky, J., Pinard, C., Pitts, S. B. J., et al. (2014). Developing an agenda for research about policies to improve access to healthy foods in rural communities: a concept mapping study. BMC public health, 14(1):1-12. 
Kelejian, H. H. and Prucha, I. R. (2010). Specification and estimation of spatial autoregressive models with autoregressive and heteroskedastic disturbances. Journal of econometrics, 157(1):53-67.

Kreif, N., Grieve, R., Hangartner, D., Turner, A. J., Nikolova, S., and Sutton, M. (2016). Examination of the synthetic control method for evaluating health policies with multiple treated units. Health economics, 25(12):1514-1528.

La Porta, R., Lopez-de Silanes, F., Shleifer, A., and Vishny, R. (1999). The Quality of Government. The Journal of Law, Economics, and Organization, 15(1):222-279.

Lark, T. J., Salmon, J. M., and Gibbs, H. K. (2015). Cropland expansion outpaces agricultural and biofuel policies in the united states. Environmental Research Letters, 10(4):044003.

Lemi, A., Liu, L., and Wright, I. A. (2021). Location Choice Determinants of Chinese and US Firms in Africa: Does Spatial Interdependence play a role? The World Economy, n/a(n/a).

LeSage, J. and Pace, R. K. (2009). Introduction to Spatial Econometrics. Chapman and Hall/CRC.

LeSage, J. P. and Pace, R. K. (2014). The Biggest Myth in Spatial Econometrics. Econometrics, 2(4):217-249.

Li, Y., Miao, R., and Khanna, M. (2019). Effects of ethanol plant proximity and crop prices on land-use change in the united states. American Journal of Agricultural Economics, 101(2):467-491.

Liou, R.-S. and Rao-Nicholson, R. (2017). Out of africa: The role of institutional distance and host-home colonial tie in south african firms' post-acquisition performance in developed economies. International Business Review, 26(6):1184-1195.

Lundan, S. M. and Jones, G. (2001). The 'commonwealth effect'and the process of internationalisation. World Economy, 24(1):99-118. 
Mactaggart, F., McDermott, L., Tynan, A., and Gericke, C. (2016). Examining health and well-being outcomes associated with mining activity in rural communities of high-income countries: A systematic review. Australian Journal of Rural Health, 24(4):230-237.

Madden, P. (2019). Figure of the week: Foreign direct investment in africa. Brookings Institution, 9 .

Makino, S. and Tsang, E. W. (2011). Historical ties and foreign direct investment: An exploratory study.

Marasteanu, I. J. and Jaenicke, E. C. (2019). Economic impact of organic agriculture hotspots in the united states. Renewable Agriculture and Food Systems, 34(6):501522.

Marcus, J. (2013). The effect of unemployment on the mental health of spousesevidence from plant closures in germany. Journal of health economics, 32(3):546558.

Motamed, M., McPhail, L., and Williams, R. (2016). Corn area response to local ethanol markets in the united states: A grid cell level analysis. American Journal of Agricultural Economics, 98(3):726-743.

Neuenkirch, M. and Neumeier, F. (2016). The impact of us sanctions on poverty. Journal of Development Economics, 121:110-119.

Nunn, N. (2007). Historical Legacies: A Model Linking Africa's Past to its Current Underdevelopment. Journal of Development Economics, 83(1):157-175.

Nwaogu, U. G. and Ryan, M. (2014). Spatial Interdependence in US Outward FDI into Africa, Latin America and the Caribbean. The World Economy, 37(9):12671289.

Pesaran, M. H. (2015). Testing Weak Cross-sectional Dependence in Large Panels. Econometric Reviews, 34(6-10):1089-1117. 
Phillips, A. Z. and Rodriguez, H. P. (2020). Us county "food swamp" severity and hospitalization rates among adults with diabetes: A nonlinear relationship. Social Science E Medicine, 249:112858.

Poelhekke, S. and Van Der Ploeg, F. (2013). Do Natural Resources Attract Nonresource FDI? Review of Economics and Statistics, 95(3):1047-1065.

Proost, S. and Thisse, J.-F. (2019). What can be learned from spatial economics? Journal of Economic Literature, 57(3):575-643.

Regelink, M. and Elhorst, J. (2015). The Spatial Econometrics of FDI and Third Country Effects. Letters in Spatial and Resource Sciences, 8(1):1-13.

Roberts, M. J. and Schlenker, W. (2013). Identifying supply and demand elasticities of agricultural commodities: Implications for the us ethanol mandate. American Economic Review, 103(6):2265-95.

Santiso, J. (2008). The Emergence of Latin Multinationals. Cepal Review, (95):7-30.

Searchinger, T., Heimlich, R., Houghton, R. A., Dong, F., Elobeid, A., Fabiosa, J., Tokgoz, S., Hayes, D., and Yu, T.-H. (2008). Use of us croplands for biofuels increases greenhouse gases through emissions from land-use change. Science, 319(5867):1238-1240.

Secchi, S., Kurkalova, L., Gassman, P. W., and Hart, C. (2011). Land use change in a biofuels hotspot: the case of iowa, usa. Biomass and Bioenergy, 35(6):2391-2400.

Sharkey, J. R., Dean, W. R., Nalty, C. C., and Xu, J. (2013). Convenience stores are the key food environment influence on nutrients available from household food supplies in texas border colonias. BMC public health, 13(1):1-8.

Siddiqui, A. and Iqbal, A. (2018). In Search of Spatial Interdependence of US Outbound FDI in the MENA Region. The World Economy, 41(5):1415-1436. 
Smith, J. A. and Todd, P. E. (2005). Does matching overcome lalonde's critique of nonexperimental estimators? Journal of econometrics, 125(1-2):305-353.

Sönnichsen, N. (2020). Coronavirus: Impact on the global energy industry - statistics \& facts. Statista. com.

Tang, X. (2019). Chinese manufacturing investments and knowledge transfer: a report from ethiopia. SAIS-CARI Working Paper, 3.

Towe, C. and Tra, C. I. (2013). Vegetable spirits and energy policy. American Journal of Agricultural Economics, 95(1):1-16.

Tuman, J. P. and Shirali, M. (2017a). The Political Economy of Chinese Foreign Direct Investment in Developing Areas. Foreign Policy Analysis, 13(1):154-167.

Tuman, J. P. and Shirali, M. (2017b). The Political Economy of Chinese Foreign Direct Investment in Developing Areas. Foreign Policy Analysis, 13(1):154-167.

Wright, C. K., Larson, B., Lark, T. J., and Gibbs, H. K. (2017). Recent grassland losses are concentrated around us ethanol refineries. Environmental Research Letters, 12(4):044001.

Wright, C. K. and Wimberly, M. C. (2013). Recent land use change in the western corn belt threatens grasslands and wetlands. Proceedings of the National Academy of Sciences, 110(10):4134-4139.

Wu, J. and Cho, S.-H. (2007). The effect of local land use regulations on urban development in the western united states. Regional Science and Urban Economics, 37(1):69-86. 